\title{
An efficient catalyst for Pd-catalyzed carbonylation of aryl arenesulfonates
}

Chaoxian Cai*, Nelo R. Rivera*, Jaume Balsells, Rick R. Sidler, J. Christopher McWilliams, C. Scott Shultz, Yongkui Sun

Process Research, Merck Research Laboratories, Merck \& Co., Inc., P. O. Box 2000, Rahway, NJ 07065

Supporting Information

List of Contents

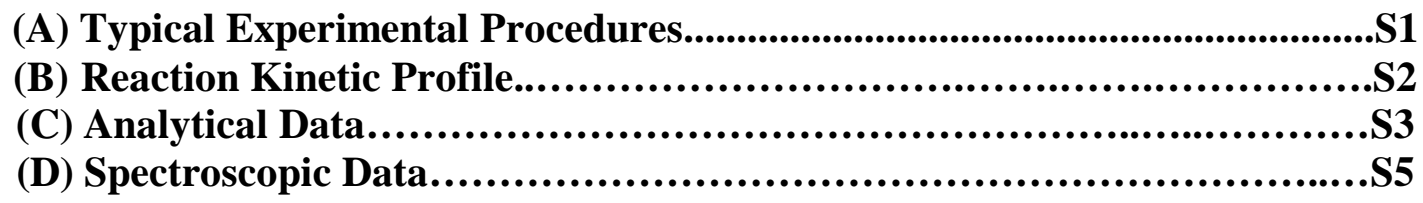




\section{Part A: Typical Experimental Procedures}

\section{General}

All chemicals except aryl p-flurobenezenesulfonates were obtained from commercial sources and used without further purification. The ferrocenyl phosphine ligands were purchased form Solvias AG. Aryl p-flurobenzenesulfonates were prepared from the corresponding phenols and $\mathrm{p}$-flurobenzenesulfonyl chloride according to the procedure in reference 4. Anhydrous solvents were used as were purchased without further distillation throughout the entire experiment. Reactions were analyzed by reversed phase HPLC, conducted on an Agilent 1100 Series instrument with DAD using a 96-well plate auto sampler. NMR experiments were conducted on a Bruker NMR (400 or $500 \mathrm{MHz}$ ) instrument.

\section{Milligram-scale screening procedure}

Symyx Technology's software and hardware were used to conduct the entire milligramscale screening experiments from library design, liquid and solid dispense, reaction heating and agitation, to HPLC analysis (http://www.symyx.com). The 96-well reactor block has the capacity to run 96 milligram-scale reactions in parallel. For all milligramscale reactions, most pipetting jobs were conducted by the Cavro robot. An organic substance was usually charged as a stock solution, and an inorganic base was dispensed as slurry, usually in dichloroethane suspension. Solid dispense by Powdernium robot was used in some cases. In a typical screening procedure, under a $\mathrm{N}_{2}$ atmosphere, a reactor block containing 96 disposable glass reaction vials $(40 \mathrm{~mm}$ height $\times 8 \mathrm{~mm}$ diameter) was dispensed with ligands $(0.44$ or $0.80 \mu \mathrm{mol})$ and $\mathrm{Pd}(\mathrm{OAc})_{2}(0.4 \mu \mathrm{mol}, 4 \mathrm{~mol} \% \mathrm{Pd}$ loading relative to p-tolyl p-flurobenezenesulfonate), and aged at room temperature for $\sim 1 \mathrm{~h}$. A ligand-to-palladium ratio of 1.1 was employed for a bidentate ligand, and 2.0 for a monodentate ligand. Ligands and $\mathrm{Pd}(\mathrm{OAc})_{2}$ were dissolved in a $1: 1$ toluene/dichloroethane mixture. p-Tolyl p-flurobenezenesulfonate $(2.66 \mathrm{mg}, 10 \mu \mathrm{mol})$ and base (2.0 equivs) were then dispensed. The reaction mixture was concentrated down in vacuum to remove all unwanted solvents from stock solutions (or slurries) prior to the addition of solvents $(200 \mu \mathrm{L})$ to be screened. The reactor block was then sealed, leak tested, and purged first by 3 times of $40 \mathrm{psi}_{2}$, followed by 3 times 40 psi CO before it was pressurized to $90 \mathrm{psi} \mathrm{CO}$. The reactor block was agitated and heated at desired temperature for certain hours. An internal standard (4,4-dimethylbiphenyl) was added to the reaction mixture for the calculations of HPLC assay yields.

\section{Gram-scale autoclave procedure}

To a $50 \mathrm{~mL}$ autoclave were charged with an aryl arenesulfonate $(\sim 1.0 \mathrm{~g}, 4 \mathrm{mmol})$, $\mathrm{NaOAc}(0.7 \mathrm{~g}, 8 \mathrm{mmol})$, and $20 \mathrm{~mL} \mathrm{EtOH}(\sim 100 \mathrm{ppm}$ water content). The reaction mixture was then mechanically stirred at $200 \mathrm{rpm}$ and degassed with $40 \mathrm{psi}_{2}$ three times. Under a $\mathrm{N}_{2}$ atmosphere, $\mathrm{Pd}(\mathrm{OAc})_{2}(36 \mathrm{mg}, 0.16 \mathrm{mmol})$, Josiphos $\mathrm{Et}_{2} \mathrm{P}-\mathrm{Fc}-\mathrm{PtBu}_{2}$ (90 $\mathrm{mg}, 0.17 \mathrm{mmol}$ ) and $2 \mathrm{~mL}$ DCE were added to an $8 \mathrm{~mL}$ vial, and aged at room

temperature for more than 1 hour. The catalyst solution was added to the autoclave, and 
the vessel was purged with 40 psi CO three times, and then pressurized with 90 psi CO. The reaction mixture was stirred at $1000 \mathrm{rpm}$ while heating to $130{ }^{\circ} \mathrm{C}$ over 30 minutes. The reaction was aged at $130{ }^{\circ} \mathrm{C}$ overnight. After completion, the reaction mixture was first filtered to remove inorganic solid, then concentrated to remove solvents. The residue was subjected to flash chromatography on silica gel to purify the product.

\section{Part B: Reaction Kinetic Profile}

The reaction progress can be monitored by in situ IR. A Mettler Toledo MultiMax ReactIR instrument was used for this task. An example of reaction kinetic profile is plotted in Figure 1. The initial temperature profile was indicated in the plot. The reaction did not proceed at an appreciable rate at temperatures below $120{ }^{\circ} \mathrm{C}$.

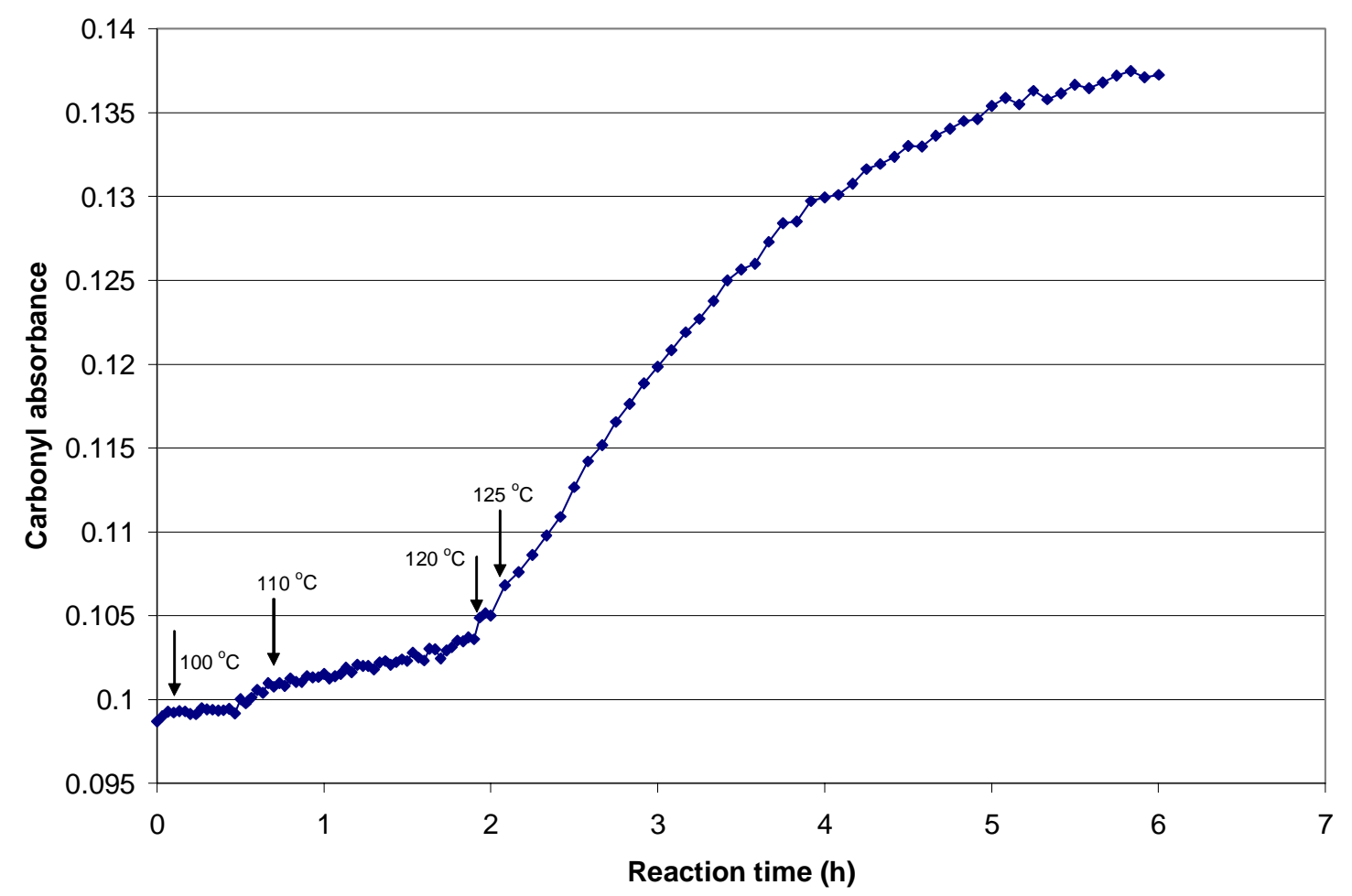

Figure 1. Carbonyl absorbance of 1-heptyl 4-methylbenzoate vs. reaction time at 1722 $\mathrm{cm}^{-1}$. Reaction temperature was ramped from r.t. to $100{ }^{\circ} \mathrm{C}$ in $15 \mathrm{~min}$, held for $20 \mathrm{~min}$; then to $110{ }^{\circ} \mathrm{C}$ in $5 \mathrm{~min}$, held for $1 \mathrm{~h}$; then to $120{ }^{\circ} \mathrm{C}$ in $5 \mathrm{~min}$, held for $10 \mathrm{~min}$; then to $125^{\circ} \mathrm{C}$ in $5 \mathrm{~min}$. Other conditions same as Table 1 . The data was recorded by subtracting baseline absorbance at $1706 \mathrm{~cm}^{-1}$. 


\section{Part C: Analytical Data}

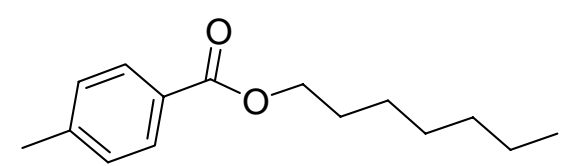

1-heptyl 4-methylbenzoate (1): ${ }^{1} \mathrm{H}$ NMR (400 MHz, $\left.\mathrm{CDCl}_{3}\right): \delta 7.94(\mathrm{~d}, 2 \mathrm{H}, \mathrm{J}=8.4$ ), $7.24(\mathrm{~d}, 2 \mathrm{H}, \mathrm{J}=8.1), 4.31(\mathrm{t}, 2 \mathrm{H}, \mathrm{J}=6.7), 2.42(\mathrm{~s}, 3 \mathrm{H}), 1.77(\mathrm{p}, 2 \mathrm{H}, \mathrm{J}=7.0), 1.27-1.48$ $(\mathrm{m}, 8 \mathrm{H}), 0.90(\mathrm{t}, 3 \mathrm{H}, \mathrm{J}=6.7) ;{ }^{13} \mathrm{C} \mathrm{NMR}\left(100 \mathrm{MHz}, \mathrm{CDCl}_{3}\right): \delta 167.0,143.6,129.8,129.2$, 128.0, 65.2, 32.0, 29.2, 29.0, 26.2, 22.8, 21.8, 14.3; HRMS (ESI): $[\mathrm{M}+\mathrm{H}]^{+}$calcd 235.16926 , found 235.17031 .

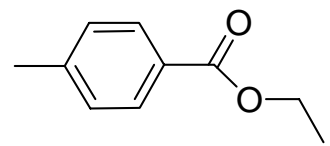

ethyl 4-methylbenzoate (2): ${ }^{1} \mathrm{H}$ NMR (400 $\mathrm{MHz}, \mathrm{CDCl}_{3}$ ): $\delta 7.94$ (dt, $2 \mathrm{H}, \mathrm{J}=8.4,2.0$ ), 7.23 (d, 2H, J = 8.8), $4.36(\mathrm{q}, 2 \mathrm{H}, \mathrm{J}=7.1), 2.40(\mathrm{~s}, 3 \mathrm{H}), 1.39(\mathrm{t}, 3 \mathrm{H}, \mathrm{J}=7.2) ;{ }^{13} \mathrm{C} \mathrm{NMR}$ $\left(100 \mathrm{MHz}, \mathrm{CDCl}_{3}\right): \delta 166.8,143.5,129.7,129.1,128.0,60.8,21.7,14.5$.<smiles>CCOC(=O)c1ccc(C(C)=O)cc1</smiles>

ethyl 4-acetobenzoate (3) : ${ }^{1} \mathrm{H} \mathrm{NMR}\left(400 \mathrm{MHz}, \mathrm{CDCl}_{3}\right): \delta 8.13(\mathrm{~d}, 2 \mathrm{H}, \mathrm{J}=8.4), 8.00$ $(\mathrm{d}, 2 \mathrm{H}, \mathrm{J}=8.4), 4.41(\mathrm{q}, 2 \mathrm{H}, \mathrm{J}=7.1), 2.65(\mathrm{~s}, 3 \mathrm{H}), 1.42(\mathrm{t}, 3 \mathrm{H}, \mathrm{J}=7.2) ;{ }^{13} \mathrm{C}$ NMR (100 $\left.\mathrm{MHz} \mathrm{CDCl}_{3}\right): \delta 197.7,165.9,140.4,134.5,130.0,128.4,61.7,27.1,14.5$.<smiles>CCOC(=O)c1ccccc1</smiles>

ethyl benzoate (4): ${ }^{1} \mathrm{H} \mathrm{NMR}\left(400 \mathrm{MHz}, \mathrm{CDCl}_{3}\right): \delta 8.06(\mathrm{dd}, 2 \mathrm{H}, \mathrm{J}=8.8,1.6), 7.56(\mathrm{tt}$, $1 \mathrm{H}, \mathrm{J}=7.4,1.2), 7.44(\mathrm{t}, 2 \mathrm{H}, \mathrm{J}=7.6), 4.39(\mathrm{q}, 2 \mathrm{H}, \mathrm{J}=7.1), 1.41(\mathrm{t}, 3 \mathrm{H}, \mathrm{J}=7.1) ;{ }^{13} \mathrm{C}$ NMR (100 MHz, $\left.\mathrm{CDCl}_{3}\right): \delta 166.9,133.0,130.7,129.7,128.5,61.1,14.5$.<smiles>CCOC(=O)c1ccccc1C</smiles>

ethyl 2-methylbenzoate (5): ${ }^{1} \mathrm{H} \mathrm{NMR}\left(400 \mathrm{MHz}, \mathrm{CDCl}_{3}\right): \delta 7.92(\mathrm{dd}, 1 \mathrm{H}, \mathrm{J}=8.4,2.0)$, $7.40(\mathrm{dt}, 1 \mathrm{H}, \mathrm{J}=7.4,1.6), 7.25(\mathrm{~m}, 2 \mathrm{H}), 4.37(\mathrm{q}, 2 \mathrm{H}, \mathrm{J}=7.1), 2.61(\mathrm{~s}, 3 \mathrm{H}), 1.41(\mathrm{t}, 3 \mathrm{H}, \mathrm{J}$ $=7.0) ;{ }^{13} \mathrm{C} \mathrm{NMR}\left(100 \mathrm{MHz}, \mathrm{CDCl}_{3}\right): \delta 167.9,140.2,132.0,131.8,130.7,130.2,125.9$, 60.9, 21.9, 14.5 . 


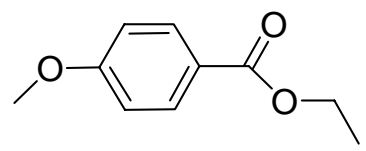

ethyl 4-methoxybenzoate (6): ${ }^{1} \mathrm{H} \mathrm{NMR}\left(400 \mathrm{MHz}, \mathrm{CDCl}_{3}\right): \delta 8.00(\mathrm{dt}, 2 \mathrm{H}, \mathrm{J}=8.8,2.0)$, $6.92(\mathrm{dt}, 2 \mathrm{H}, \mathrm{J}=8.4,2.0), 4.35$ (q, 2H, J = 7.1), 3.86 (s, 3H), $1.38(\mathrm{t}, 3 \mathrm{H}, 7.2) ;{ }^{13} \mathrm{C} \mathrm{NMR}$ $\left(100 \mathrm{MHz}, \mathrm{CDCl}_{3}\right): \delta 166.6,163.5,131.7,123.2,113.7,60.8,55.6,14.6$.

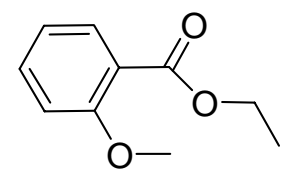

ethyl 2-methoxybenzoate (7): ${ }^{1} \mathrm{H} \mathrm{NMR}\left(400 \mathrm{MHz}, \mathrm{CDCl}_{3}\right): \delta 7.78(\mathrm{dd}, 1 \mathrm{H}, \mathrm{J}=8.0,1.8)$, 7.45 (ddd, 1H, J = 7.8, 7.2, 2.0), 6.97 (m, 2H), 4.36 (q, 2H, J = 7.2), 3.90 (s, 3H), 1.38 (t, $3 \mathrm{H}, \mathrm{J}=7.0) ;{ }^{13} \mathrm{C}$ NMR $\left(100 \mathrm{MHz}, \mathrm{CDCl}_{3}\right): \delta 166.4,159.3,133.5,131.6,120.7,120.3$, $112.2,60.9,56.1,14.5$.

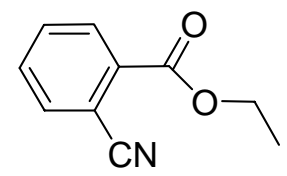

ethyl 2-cyanobenzoate (8): ${ }^{1} \mathrm{H} \mathrm{NMR}\left(400 \mathrm{MHz}, \mathrm{CDCl}_{3}\right): \delta 8.11(\mathrm{dd}, 1 \mathrm{H}, \mathrm{J}=6.8,2.0)$, 7.77 (dd, $1 \mathrm{H}, \mathrm{J}=7.2,2.0), 7.65(\mathrm{~m}, 2 \mathrm{H}), 4.44(\mathrm{q}, 2 \mathrm{H}, \mathrm{J}=7.2), 1.42(\mathrm{t}, 3 \mathrm{H}, \mathrm{J}=7.0) ;{ }^{13} \mathrm{C}$ NMR (100 MHz, $\left.\mathrm{CDCl}_{3}\right): \delta 164.1,134.8,132.8,132.7,132.6,131.2,117.6,113.0,62.3$, 14.2 .

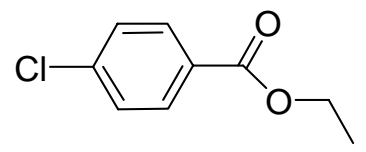

ethyl 4-chlorobenzoate (9): ${ }^{1} \mathrm{H} \mathrm{NMR}\left(400 \mathrm{MHz}, \mathrm{CDCl}_{3}\right): \delta 7.96(\mathrm{dt}, 2 \mathrm{H}, \mathrm{J}=8.8,2.0)$, 7.39 (dt, 2H, J = 8.8, 2.0), 4.36 (q, 2H, J = 7.2), 1.38 (t, 3H, J = 7.0); ${ }^{13} \mathrm{C}$ NMR (100 MHz, $\left.\mathrm{CDCl}_{3}\right): \delta 165.9,139.4,131.1,129.1,128.8,61.3,14.4$.<smiles>CCOC(=O)c1ccc(C(=O)OCC)cc1</smiles>

diethyl terephthalate (10): ${ }^{1} \mathrm{H}$ NMR $\left(400 \mathrm{MHz} \mathrm{CDCl}_{3}\right): \delta 8.10(\mathrm{~s}, 4 \mathrm{H}), 4.41(\mathrm{q}, 4 \mathrm{H}, \mathrm{J}=$ 7.3), 1.41 (t, $6 \mathrm{H}, \mathrm{J}=7.0) ;{ }^{13} \mathrm{C} \mathrm{NMR}\left(100 \mathrm{MHz}, \mathrm{CDCl}_{3}\right): \delta 166.0,134.4,129.7,61.6,14.5$.<smiles>CCOC(=O)c1ccc(F)cc1</smiles>

ethyl 4-fluorobenzoate (11): ${ }^{1} \mathrm{H}$ NMR (400 $\left.\mathrm{MHz}, \mathrm{CDCl}_{3}\right): \delta 8.05$ (dd, $\left.2 \mathrm{H}, \mathrm{J}=8.8,5.4\right)$, 7.10 (tt, $2 \mathrm{H}, \mathrm{J}=8.6,1.8), 4.37$ (q, 2H, J = 7.1), $1.39(\mathrm{t}, 3 \mathrm{H}, \mathrm{J}=7.0) ;{ }^{13} \mathrm{C} \mathrm{NMR}(100 \mathrm{MHz}$, $\left.\mathrm{CDCl}_{3}\right): \delta 165.9\left(\mathrm{~d}, \mathrm{~J}_{\mathrm{C}-\mathrm{F}}=253.4\right), 165.8,132.2\left(\mathrm{~d}, \mathrm{~J}_{\mathrm{C}-\mathrm{F}}=9.4\right), 126.9\left(\mathrm{~d}, \mathrm{~J}_{\mathrm{C}-\mathrm{F}}=2.9\right), 115.6$ $\left(\mathrm{d}, \mathrm{J}_{\mathrm{C}-\mathrm{F}}=22.1\right), 61.2,14.5$. 


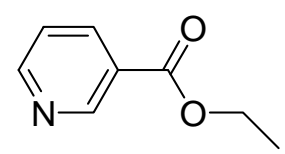

ethyl nicotinate (12): ${ }^{1} \mathrm{H}$ NMR $\left(400 \mathrm{MHz}, \mathrm{CDCl}_{3}\right): \delta 9.17(\mathrm{~d}, 1 \mathrm{H}, \mathrm{J}=1.6), 8.71(\mathrm{dd}, 1 \mathrm{H}$, $\mathrm{J}=4.8,1.6), 8.24(\mathrm{dt}, 1 \mathrm{H}, \mathrm{J}=8.4,2.4), 7.33(\mathrm{ddd}, 1 \mathrm{H}, \mathrm{J}=8.0,4.8,0.8), 4.36(\mathrm{q}, 2 \mathrm{H}, \mathrm{J}=$ 7.1), $1.35(\mathrm{t}, 3 \mathrm{H}, \mathrm{J}=7.2) ;{ }^{13} \mathrm{C}$ NMR $\left(100 \mathrm{MHz}, \mathrm{CDCl}_{3}\right): \delta 165.3,153.4,150.9,137.1$, $126.4,123.3,61.5,14.3$.

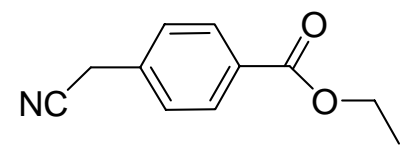

ethyl 4-cyanomethylbenzoate (13): ${ }^{1} \mathrm{H} \mathrm{NMR}\left(400 \mathrm{MHz}, \mathrm{CDCl}_{3}\right): \delta 8.06(\mathrm{dt}, 2 \mathrm{H}, \mathrm{J}=8.4$, 2.0), $7.42(\mathrm{dd}, 2 \mathrm{H}, \mathrm{J}=8.0,0.8), 4.39(\mathrm{q}, 2 \mathrm{H}, \mathrm{J}=7.2), 3.82(\mathrm{~s}, 2 \mathrm{H}), 1.41(\mathrm{t}, 3 \mathrm{H}, \mathrm{J}=7.1)$; ${ }^{13} \mathrm{C} \mathrm{NMR}\left(100 \mathrm{MHz}, \mathrm{CDCl}_{3}\right): \delta 166.1,134.9,130.7,130.6,128.1,117.3,61.4,23.9,14.5$.<smiles>CC(=O)c1ccc(OS(=O)(=O)c2ccc(F)cc2)cc1</smiles>

4-acetylphenyl 4-fluorobenzenesulfonate (14): ${ }^{1} \mathrm{H} \mathrm{NMR}\left(400 \mathrm{MHz}, \mathrm{CDCl}_{3}\right): \delta 7.93(\mathrm{~m}$, 4H), 7.23 (m, 2H), $7.11(\mathrm{~m}, 2 \mathrm{H}), 2.59(\mathrm{~s}, 3 \mathrm{H}) ;{ }^{13} \mathrm{C} \mathrm{NMR}\left(100 \mathrm{MHz}, \mathrm{CDCl}_{3}\right): \delta$ 196.7, $166.4\left(\mathrm{~d}, J_{\mathrm{C}-\mathrm{F}}=258.2\right), 152.9,136.1,131.6\left(\mathrm{~d}, J_{\mathrm{C}-\mathrm{F}}=9.7\right), 131.3\left(\mathrm{~d}, J_{\mathrm{C}-\mathrm{F}}=3.3\right), 130.3$, $122.6,117.0\left(\mathrm{~d}, J_{\mathrm{C}-\mathrm{F}}=22.7\right), 26.8$.<smiles>O=S(=O)(Oc1ccccc1)c1ccc(F)cc1</smiles>

phenyl 4-fluorobenzenesulfonate (15): ${ }^{1} \mathrm{H}$ NMR (400 MHz, $\left.\mathrm{CDCl}_{3}\right): \delta 7.85(\mathrm{~m}, 2 \mathrm{H})$, $7.30(\mathrm{~m}, 3 \mathrm{H}), 7.20(\mathrm{~m}, 2 \mathrm{H}), 6.99(\mathrm{~m}, 2 \mathrm{H}) ;{ }^{13} \mathrm{C} \mathrm{NMR}\left(100 \mathrm{MHz}, \mathrm{CDCl}_{3}\right) \delta 166.2\left(\mathrm{~d}, J_{\mathrm{C}-\mathrm{F}}=\right.$ $257.1), 149.7,131.6\left(\mathrm{~d}, J_{\mathrm{C}-\mathrm{F}}=3.5\right), 131.6\left(\mathrm{~d}, J_{\mathrm{C}-\mathrm{F}}=9.6\right), 129.9,127.5,122.5,116.7\left(\mathrm{~d}, J_{\mathrm{C}-}\right.$ $\mathrm{F}=22.6)$.

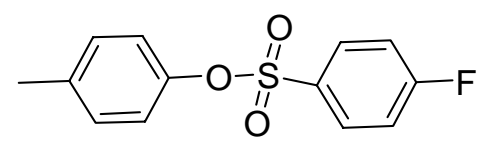

4-methylphenyl 4-fluorobenzenesulfonate (16): ${ }^{1} \mathrm{H}$ NMR (400 MHz, $\left.\mathrm{CDCl}_{3}\right): \delta 7.84$ $(\mathrm{m}, 2 \mathrm{H}), 7.21(\mathrm{~m}, 2 \mathrm{H}), 7.13(\mathrm{~m}, 2 \mathrm{H}), 6.86(\mathrm{~m}, 2 \mathrm{H}), 2.32(\mathrm{~s}, 3 \mathrm{H}) ;{ }^{13} \mathrm{C} \mathrm{NMR}(100 \mathrm{MHz}$, $\left.\mathrm{CDCl}_{3}\right) \delta 66.2\left(\mathrm{~d}, J_{\mathrm{C}-\mathrm{F}}=257.0\right), 147.5,137.4,131.6\left(\mathrm{~d}, J_{\mathrm{C}-\mathrm{F}}=4.0\right), 131.5\left(\mathrm{~d}, J_{\mathrm{C}-\mathrm{F}}=9.6\right)$, $130.4,122.2,116.7\left(\mathrm{~d}, J_{\mathrm{C}-\mathrm{F}}=22.9\right), 21.1$. 


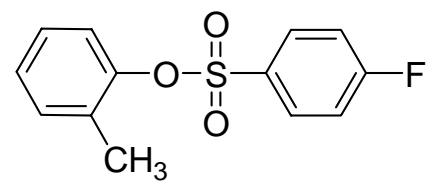

2-methylphenyl 4-fluorobenzenesulfonate (17): ${ }^{1} \mathrm{H}$ NMR (500 MHz, $\left.\mathrm{CDCl}_{3}\right): \delta 7.91$ $(\mathrm{m}, 2 \mathrm{H}), 7.19(\mathrm{~m}, 5 \mathrm{H}), 7.01(\mathrm{~m}, 1 \mathrm{H}), 2.10(\mathrm{~s}, 3 \mathrm{H}) ;{ }^{13} \mathrm{C} \mathrm{NMR}\left(125 \mathrm{MHz}, \mathrm{CDCl}_{3}\right)$ : $\delta 166.2\left(\mathrm{~d}, J_{\mathrm{C}-\mathrm{F}}=257.3\right), 148.4,132.4\left(\mathrm{~d}, J_{\mathrm{C}-\mathrm{F}}=3.3\right), 131.9,131.7,131.5\left(\mathrm{~d}, J_{\mathrm{C}-\mathrm{F}}=9.6\right)$, $127.4,127.2,122.4,116.8\left(\mathrm{~d}, J_{\mathrm{C}-\mathrm{F}}=23.6\right), 16.5$.<smiles>COc1ccc(OS(=O)(=O)c2ccc(F)cc2)cc1</smiles>

4-methoxyphenyl 4-fluorobenzenesulfonate (18): ${ }^{1} \mathrm{H}$ NMR (400 MHz, $\mathrm{CDCl}_{3}$ ): $\delta 7.83$ $(\mathrm{m}, 2 \mathrm{H}), 7.21(\mathrm{~m}, 2 \mathrm{H}), 6.89(\mathrm{~m}, 2 \mathrm{H}), 6.79(\mathrm{~m}, 2 \mathrm{H}), 3.78(\mathrm{~s}, 3 \mathrm{H}),{ }^{13} \mathrm{C}$ NMR $(100 \mathrm{MHz}$, $\left.\mathrm{CDCl}_{3}\right): \delta 166.2\left(\mathrm{~d}, J_{\mathrm{C}-\mathrm{F}}=257.2\right), 158.6,143.1,131.6\left(\mathrm{~d}, J_{\mathrm{C}-\mathrm{F}}=9.7\right), 131.5\left(\mathrm{~d}, J_{\mathrm{C}-\mathrm{F}}=3.4\right)$, $123.5,116.7\left(\mathrm{~d}, J_{\mathrm{C}-\mathrm{F}}=22.8\right), 114.8,55.8$.

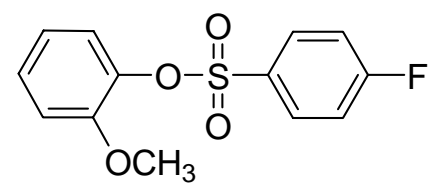

2-methoxyphenyl 4-fluorobenzenesulfonate (19): ${ }^{1} \mathrm{H} \mathrm{NMR}\left(500 \mathrm{MHz}, \mathrm{CDCl}_{3}\right): \delta 7.91$ $(\mathrm{m}, 2 \mathrm{H}), 7.22(\mathrm{~m}, 4 \mathrm{H}), 6.93(\mathrm{~m}, 1 \mathrm{H}), 6.86(\mathrm{~d}, 1 \mathrm{H}, \mathrm{J}=8.1), 3.56(\mathrm{~s}, 3 \mathrm{H}) ;{ }^{13} \mathrm{C} \mathrm{NMR}(125$ $\left.\mathrm{MHz}, \mathrm{CDCl}_{3}\right): \delta 166.1\left(\mathrm{~d}, J_{\mathrm{C}-\mathrm{F}}=256.7\right), 151.8,138.4,132.5\left(\mathrm{~d}, J_{\mathrm{C}-\mathrm{F}}=3.2\right), 131.7\left(\mathrm{~d}, J_{\mathrm{C}-\mathrm{F}}\right.$ $=9.5), 128.5,124.4,121.0,116.2\left(\mathrm{~d}, J_{\mathrm{C}-\mathrm{F}}=22.7\right), 112.9,55.7 ; \mathrm{HRMS}(\mathrm{ESI}):[\mathrm{M}+\mathrm{H}]^{+}$ calcd 283.04348, found 235.04386.

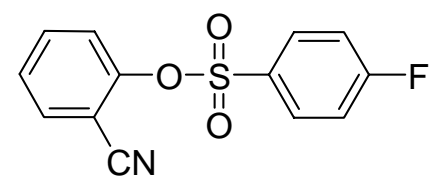

2-cyanophenyl 4-fluorobenzenesulfonate (20): ${ }^{1} \mathrm{H}$ NMR (500 MHz, $\left.\mathrm{CDCl}_{3}\right): \delta 8.00(\mathrm{~m}$, $2 \mathrm{H}), 7.68(\mathrm{~m}, 1 \mathrm{H}), 7.62(\mathrm{~m}, 1 \mathrm{H}), 7.55(\mathrm{~m}, 1 \mathrm{H}), 7.43(\mathrm{~m}, 1 \mathrm{H}), 7.28(\mathrm{~m}, 2 \mathrm{H}),{ }^{13} \mathrm{C} \mathrm{NMR}$ $\left(125 \mathrm{MHz}, \mathrm{CDCl}_{3}\right): \delta 166.8\left(\mathrm{~d}, J_{\mathrm{C}-\mathrm{F}}=258.7\right), 150.2,134.6,134.0,132.0\left(\mathrm{~d}, J_{\mathrm{C}-\mathrm{F}}=9.9\right)$, $130.7\left(\mathrm{~d}, J_{\mathrm{C}-\mathrm{F}}=3.2\right), 127.8,124.1,117.1\left(\mathrm{~d}, J_{\mathrm{C}-\mathrm{F}}=22.9\right), 114.5,107.9$.<smiles>O=[SH](O)(Oc1ccc(Cl)cc1)c1ccc(F)cc1</smiles>

4-chlorophenyl 4-fluorobenzenesulfonate (21): ${ }^{1} \mathrm{H} \mathrm{NMR}\left(400 \mathrm{MHz}, \mathrm{CDCl}_{3}\right): \delta 7.86(\mathrm{~m}$, $2 \mathrm{H}), 7.25(\mathrm{~m}, 4 \mathrm{H}), 6.94(\mathrm{~m}, 2 \mathrm{H}) ;{ }^{13} \mathrm{C} \mathrm{NMR}\left(100 \mathrm{MHz}, \mathrm{CDCl}_{3}\right): \delta 166.4\left(\mathrm{~d}, J_{\mathrm{C}-\mathrm{F}}=258.0\right)$, $148.1,133.3,131.7\left(\mathrm{~d}, J_{\mathrm{C}-\mathrm{F}}=9.8\right), 131.6\left(\mathrm{~d}, J_{\mathrm{C}-\mathrm{F}}=4.0\right), 130.1,123.9,116.9\left(\mathrm{~d}, J_{\mathrm{C}-\mathrm{F}}=\right.$ 22.7). 


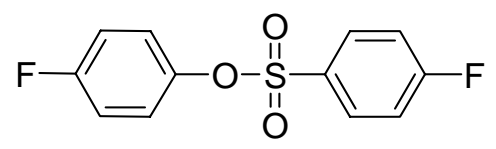

4-flurophenyl 4-fluorobenzenesulfonate (22): ${ }^{1} \mathrm{H}$ NMR (500 MHz, $\left.\mathrm{CDCl}_{3}\right): \delta 7.86(\mathrm{~m}$, $2 \mathrm{H}), 7.24(\mathrm{~m}, 2 \mathrm{H}), 6.99(\mathrm{~m}, 4 \mathrm{H}) ;{ }^{13} \mathrm{C} \mathrm{NMR}\left(125 \mathrm{MHz}, \mathrm{CDCl}_{3}\right): \delta 166.3\left(\mathrm{~d}, J_{\mathrm{C}-\mathrm{F}}=257.8\right)$, $161.4\left(\mathrm{~d}, J_{\mathrm{C}-\mathrm{F}}=247.1\right), 145.4\left(\mathrm{~d}, J_{\mathrm{C}-\mathrm{F}}=2.9\right), 131.7\left(\mathrm{~d}, J_{\mathrm{C}-\mathrm{F}}=9.7\right), 131.3\left(\mathrm{~d}, J_{\mathrm{C}-\mathrm{F}}=3.2\right)$, $124.2\left(\mathrm{~d}, J_{\mathrm{C}-\mathrm{F}}=8.7\right), 116.9\left(\mathrm{~d}, J_{\mathrm{C}-\mathrm{F}}=22.8\right), 116.7\left(\mathrm{~d}, J_{\mathrm{C}-\mathrm{F}}=23.7\right)$.<smiles>O=[SH](O)(Oc1cccnc1)c1ccc(F)cc1</smiles>

pyridin-3-yl 4-fluorobenzenesulfonate (23): ${ }^{1} \mathrm{H}$ NMR $\left(400 \mathrm{MHz}, \mathrm{CDCl}_{3}\right)$ : $\delta 8.52(\mathrm{~m}$, $1 \mathrm{H}), 8.19(\mathrm{~d}, \mathrm{~J}=2.5 \mathrm{~Hz}, 1 \mathrm{H}), 7.87(\mathrm{~m}, 2 \mathrm{H}), 7.48(\mathrm{~m}, 1 \mathrm{H}), 7.32(\mathrm{~m}, 1 \mathrm{H}), 7.24(\mathrm{~m}, 2 \mathrm{H}) ;{ }^{13} \mathrm{C}$ NMR $\left(100 \mathrm{MHz}, \mathrm{CDCl}_{3}\right): \delta 166.5\left(\mathrm{~d}, J_{\mathrm{C}-\mathrm{F}}=258.7\right), 148.7,146.5,144.1,131.6\left(\mathrm{~d}, J_{\mathrm{C}-\mathrm{F}}=\right.$ $9.7), 130.9\left(\mathrm{~d}, J_{\mathrm{C}-\mathrm{F}}=3.3\right), 130.3,124.5,117.1\left(\mathrm{~d}, J_{\mathrm{C}-\mathrm{F}}=22.8\right)$.<smiles>Cc1ccc(OS(=O)(=O)c2ccc(C)cc2)cc1</smiles>

4-methylphenyl 4-methylbenzenesulfonate (24): ${ }^{1} \mathrm{H}$ NMR $\left(400 \mathrm{MHz}, \mathrm{CDCl}_{3}\right): \delta 7.71$ $(\mathrm{m}, 2 \mathrm{H}), 7.31(\mathrm{~m}, 2 \mathrm{H}), 7.07(\mathrm{~m}, 2 \mathrm{H}), 6.86(\mathrm{~m}, 2 \mathrm{H}), 2.46(\mathrm{~s}, 3 \mathrm{H}), 2.31(\mathrm{~s}, 3 \mathrm{H}) ;{ }^{13} \mathrm{C}$ NMR $\left(125 \mathrm{MHz}, \mathrm{CDCl}_{3}\right): \delta 147.7,145.4,137.1,132.8,130.3,129.9,128.8,122.3,21.9,21.1$.

Part D: Spectroscopic Data 


\section{1-heptyl 4-methylbenzoate (1)}
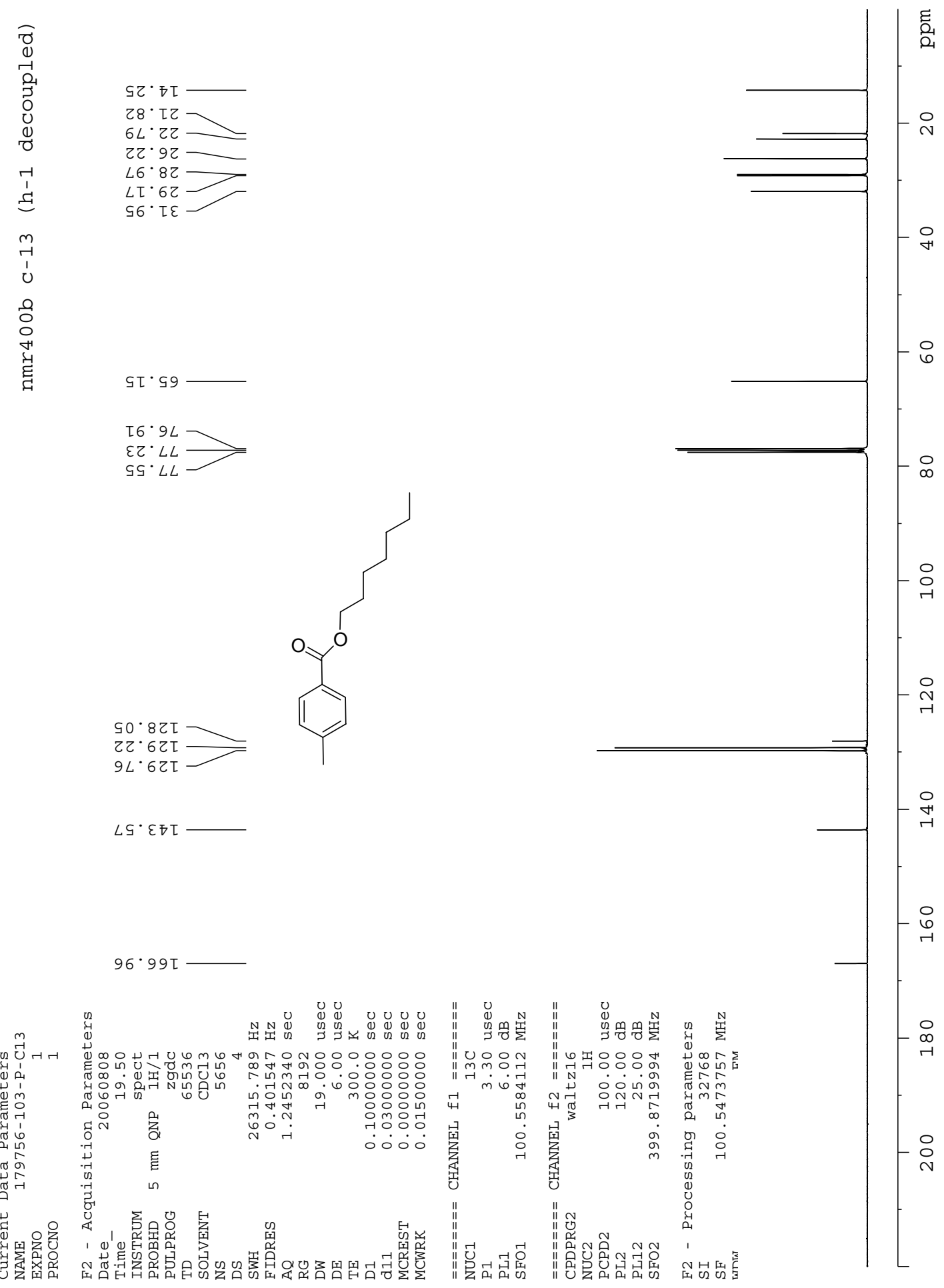


\section{1-heptyl 4-methylbenzoate (1)}
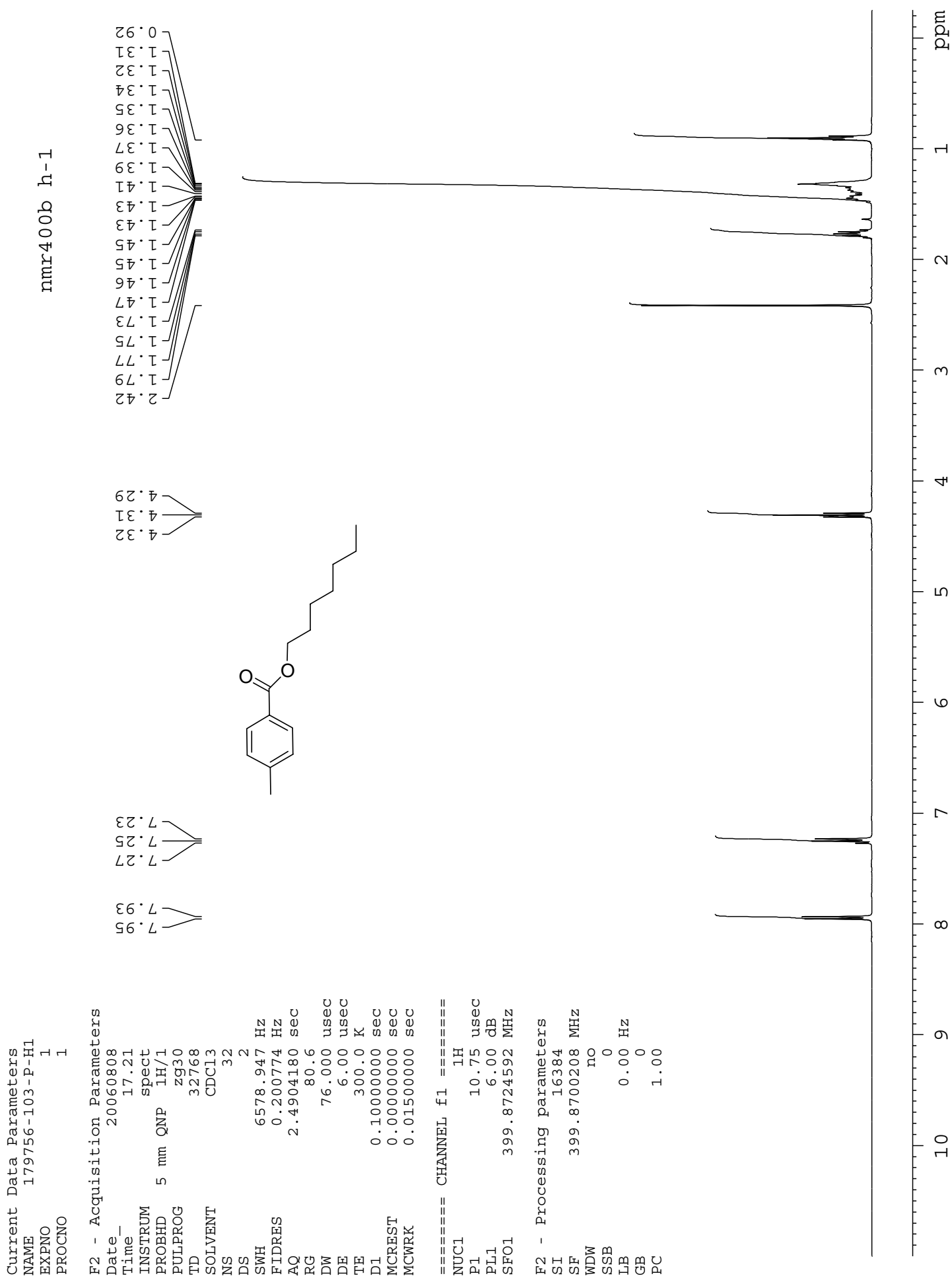
ethyl 4-methylbenzoate (2)
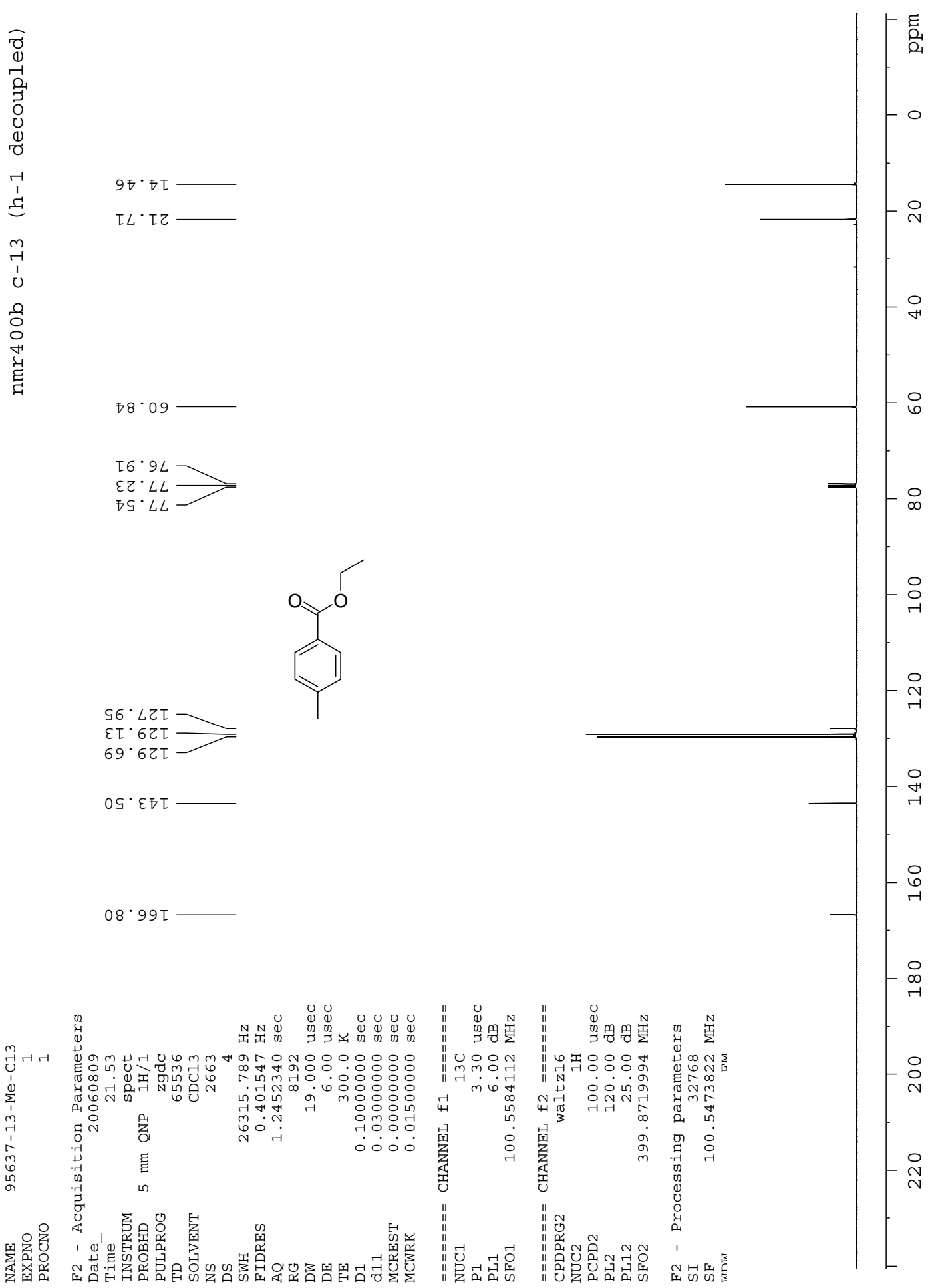
ethyl 4-methylbenzoate (2)

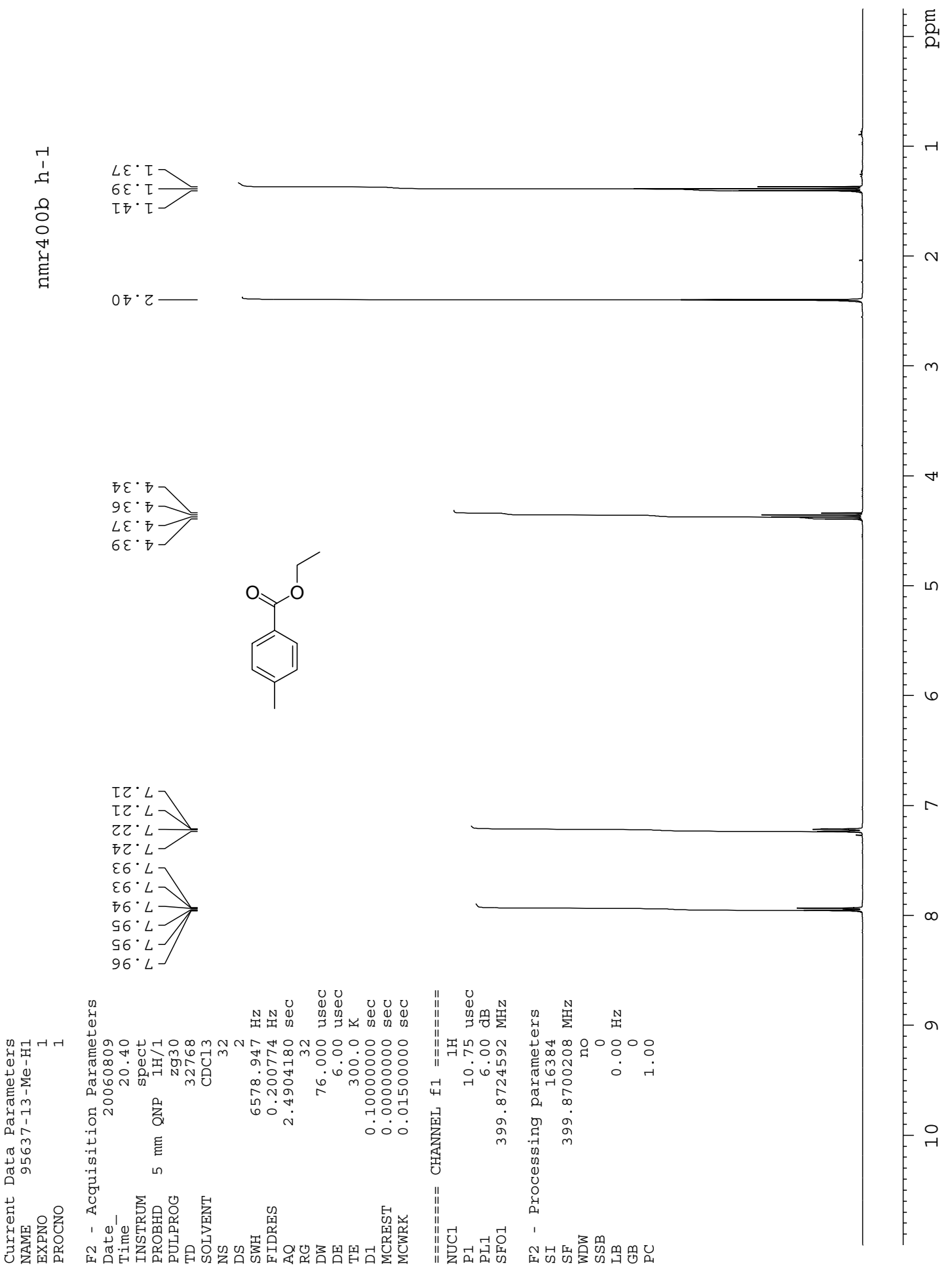


ethyl 4-acetobenzoate (3)
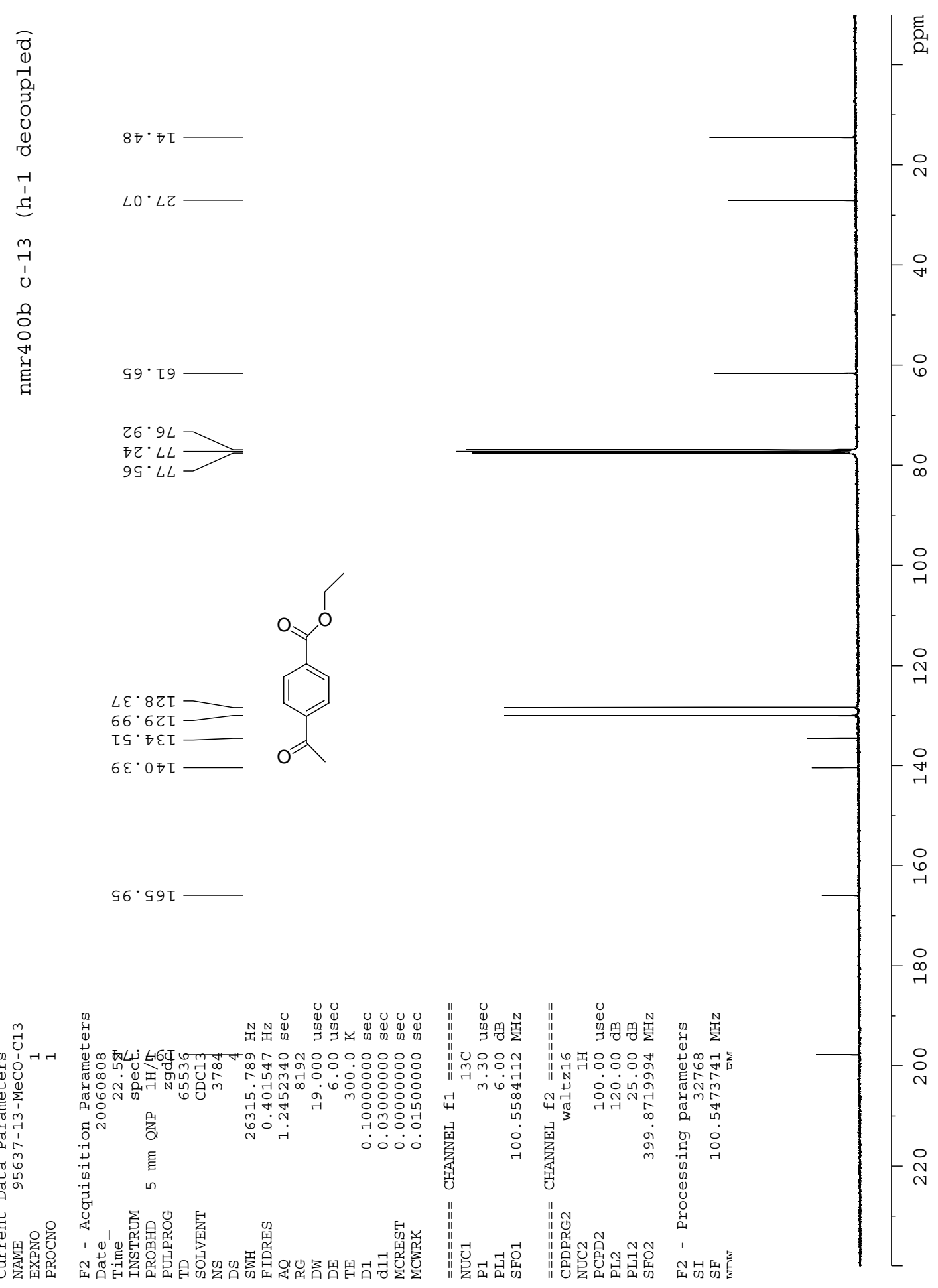
ethyl 4-acetobenzoate (3)

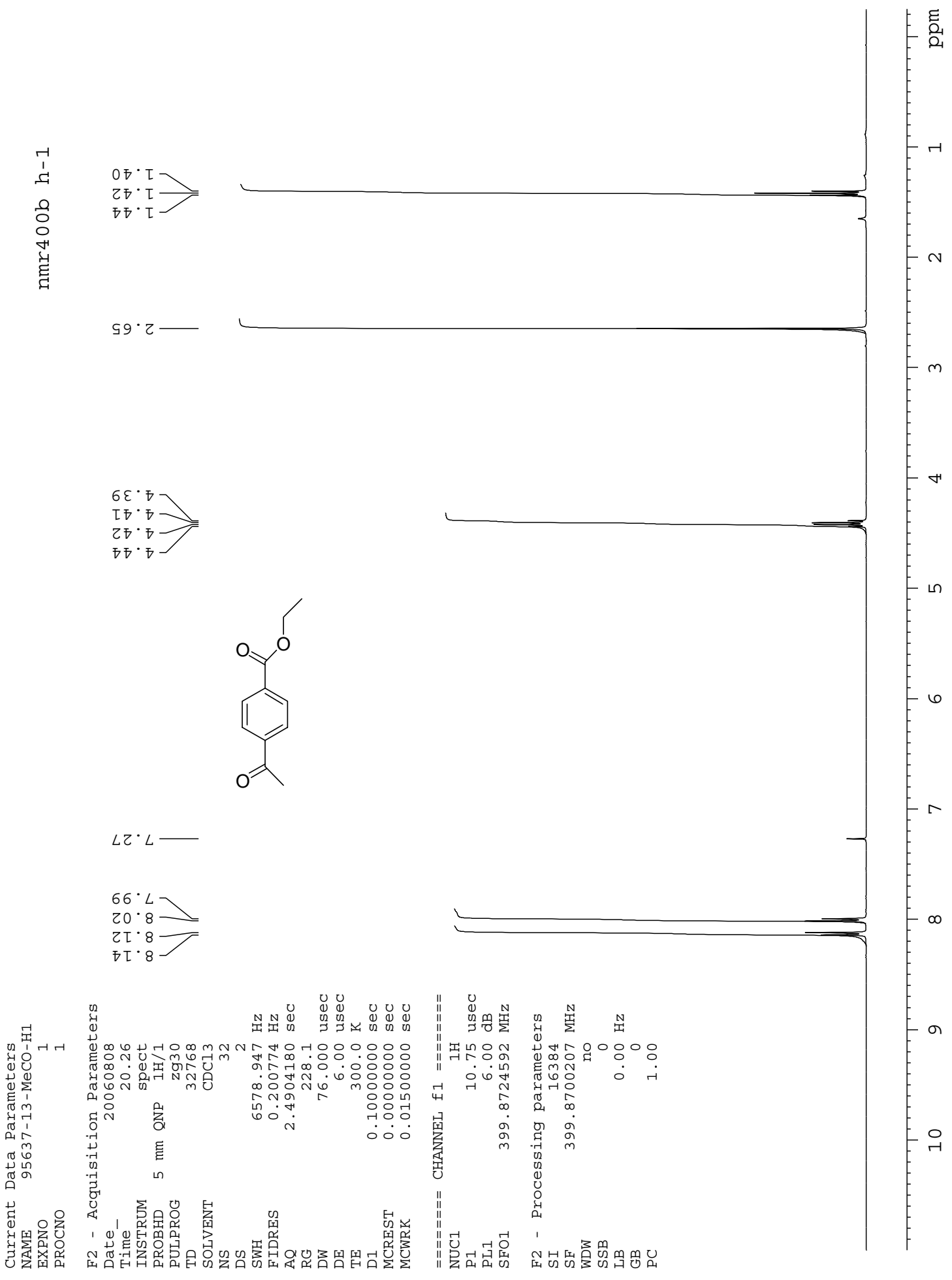


ethyl benzoate (4)
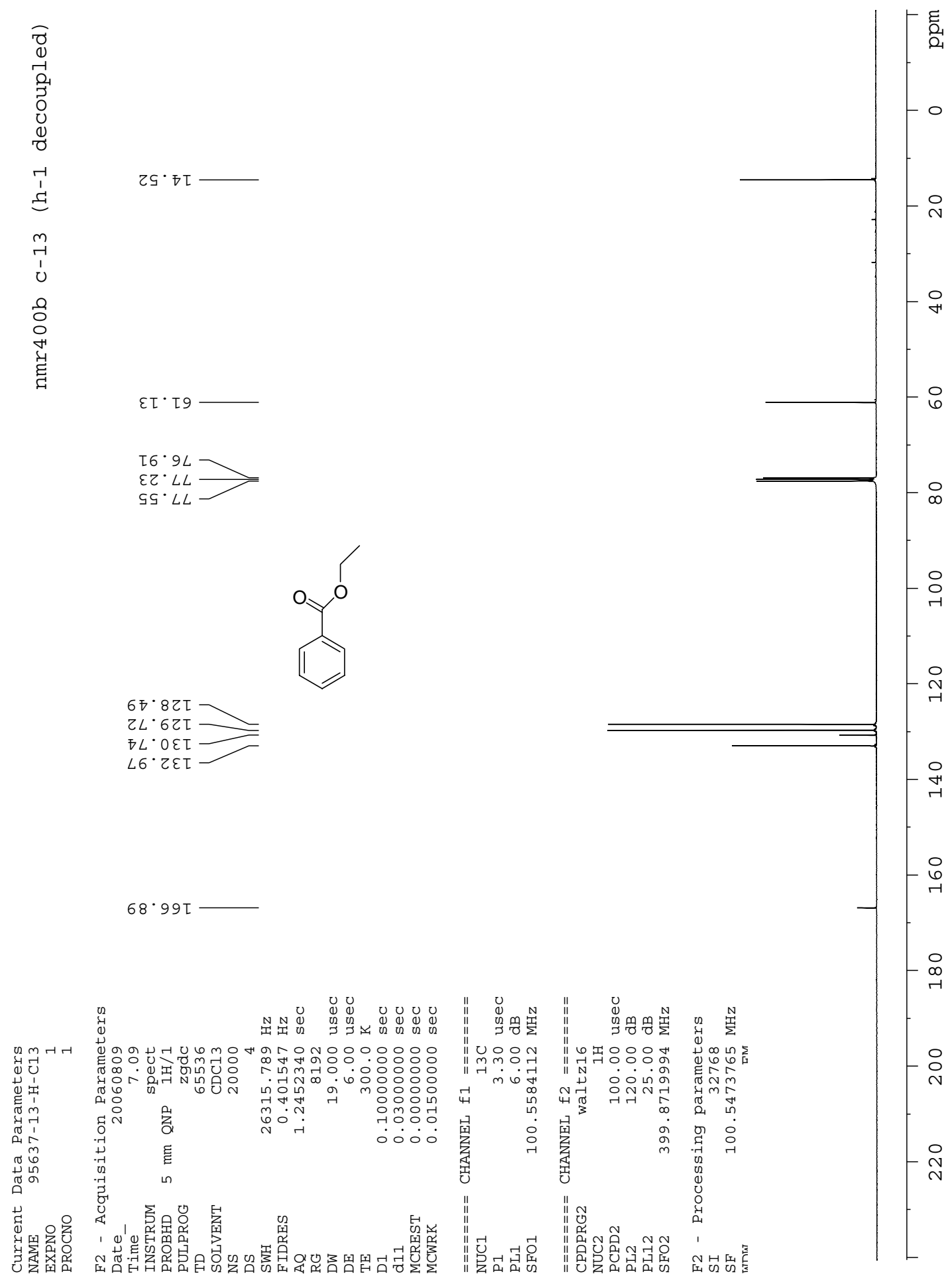
ethyl benzoate (4)

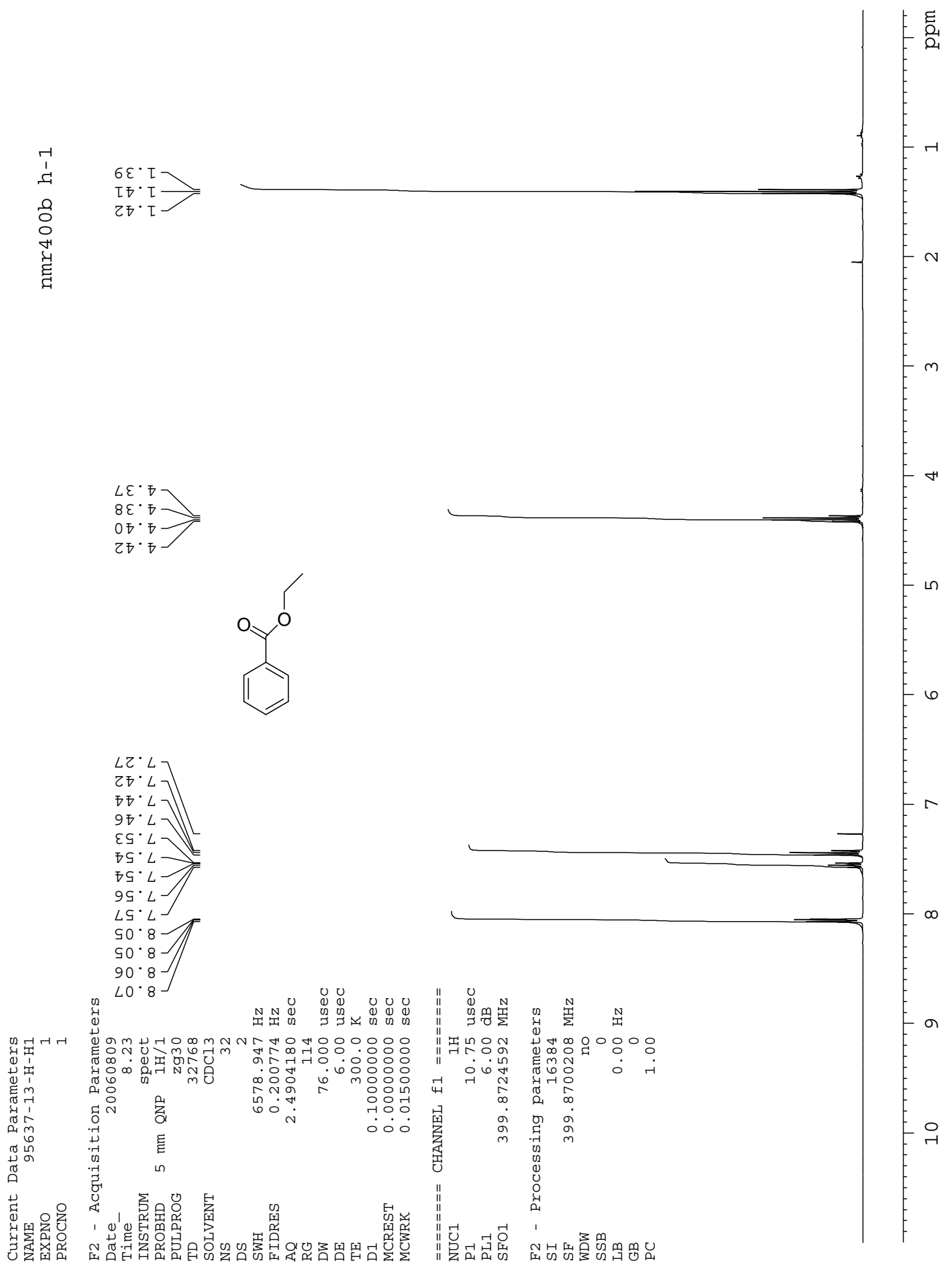


ethyl 2-methylbenzoate (5)
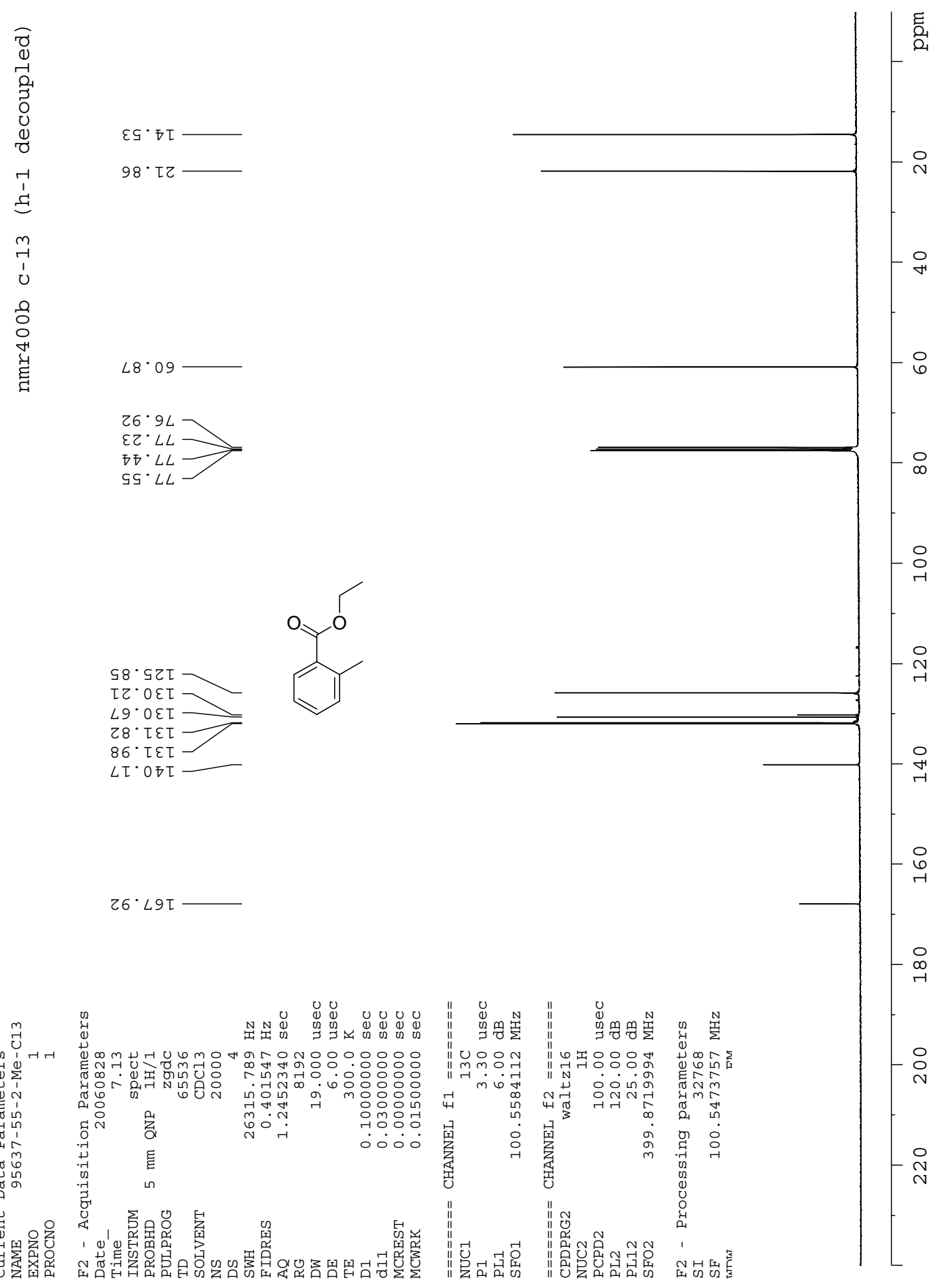


\section{ethyl 2-methylbenzoate (5)}

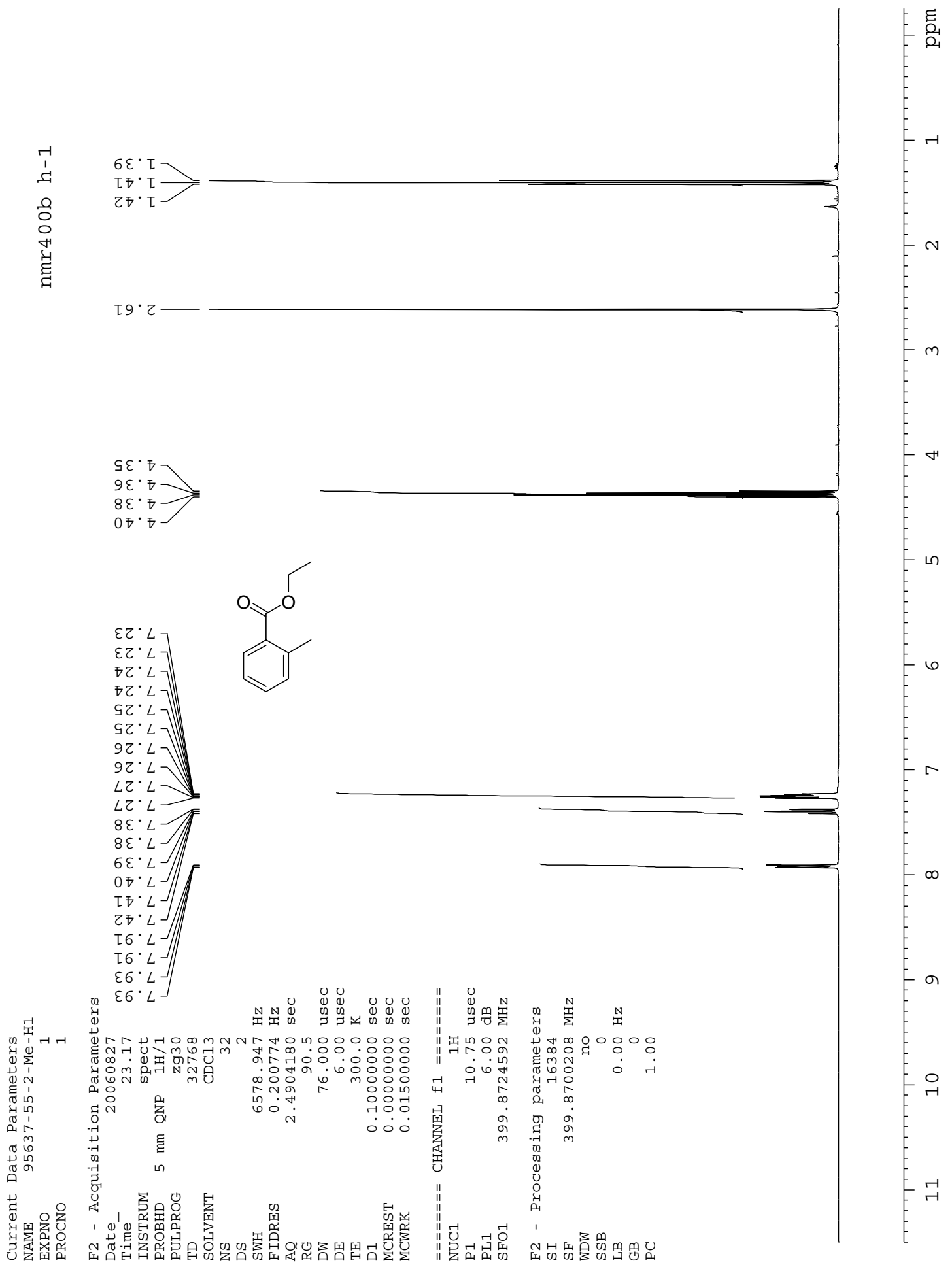


ethyl 4-methoxybenzoate (6)

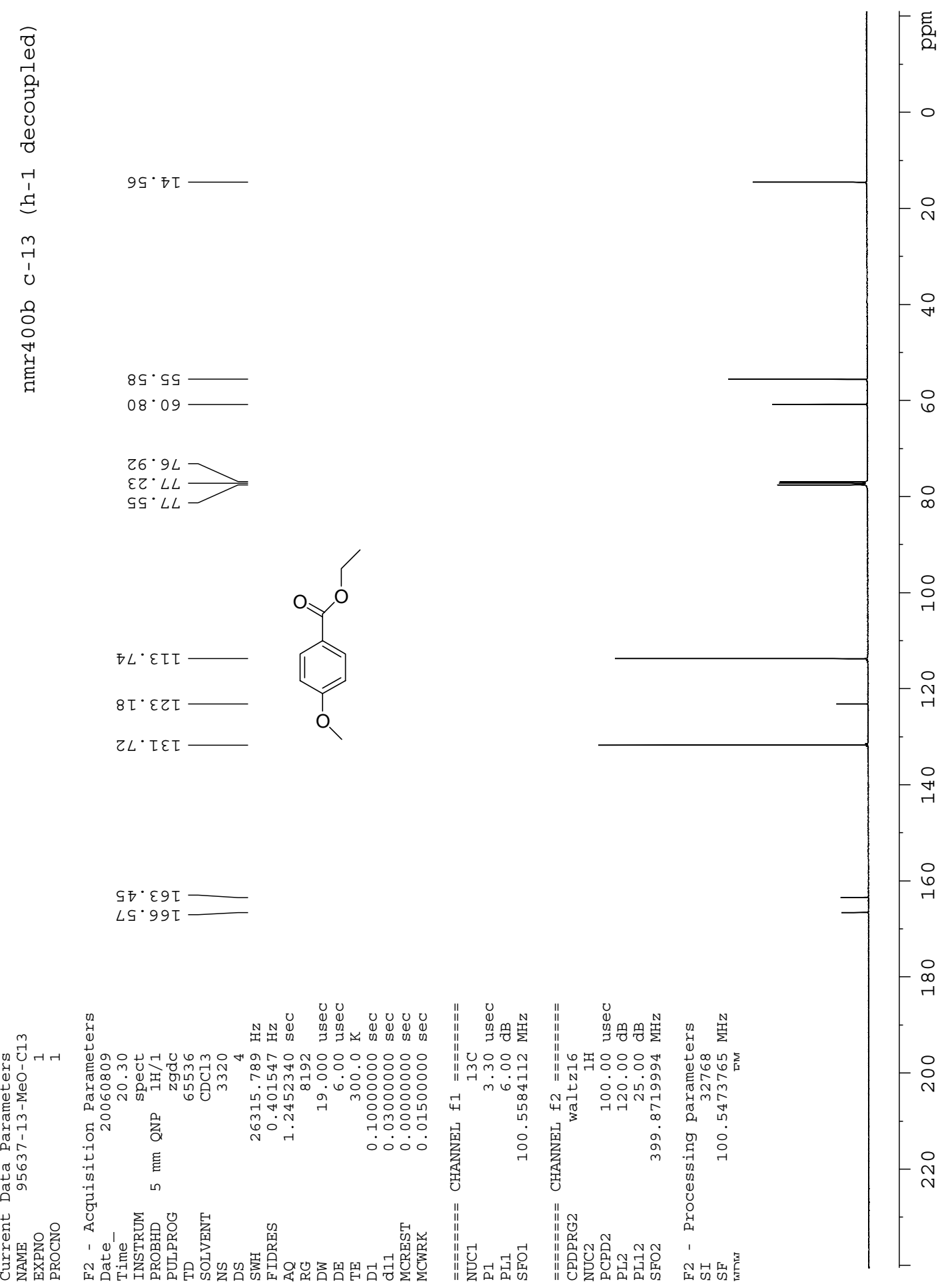


ethyl 4-methoxybenzoate (6)

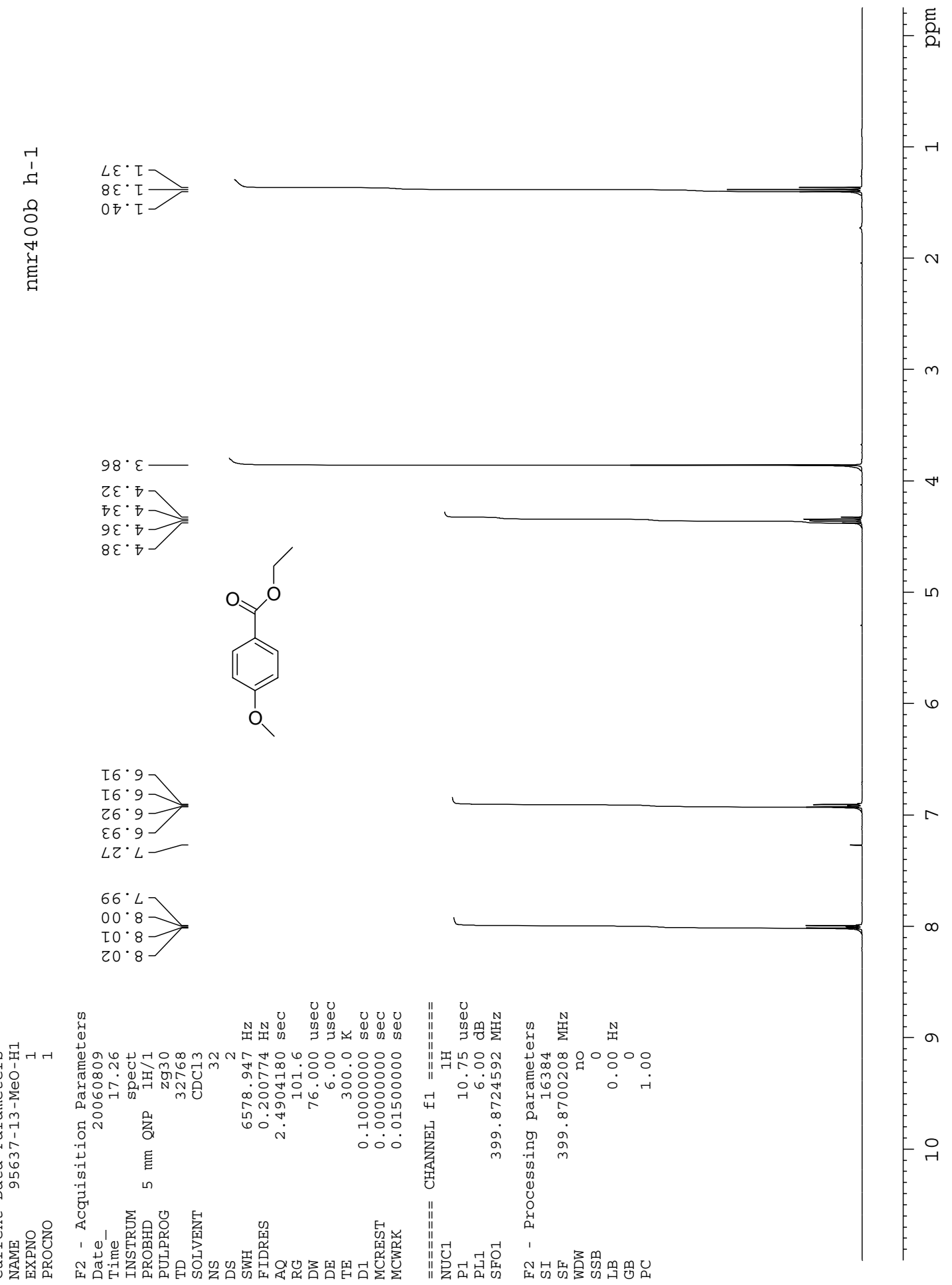


ethyl 2-methoxybenzoate (7)

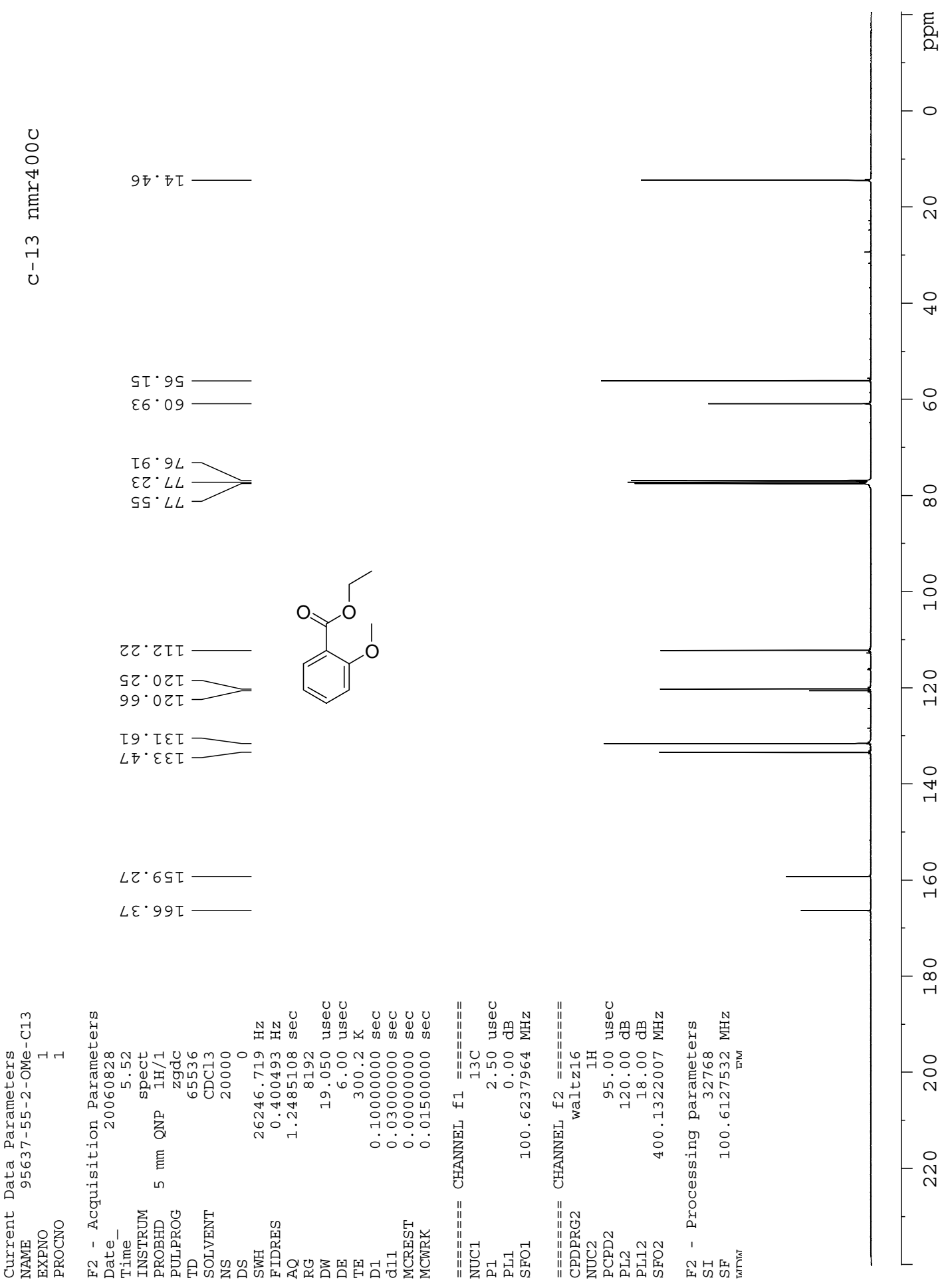




\section{ethyl 2-methoxybenzoate (7)}

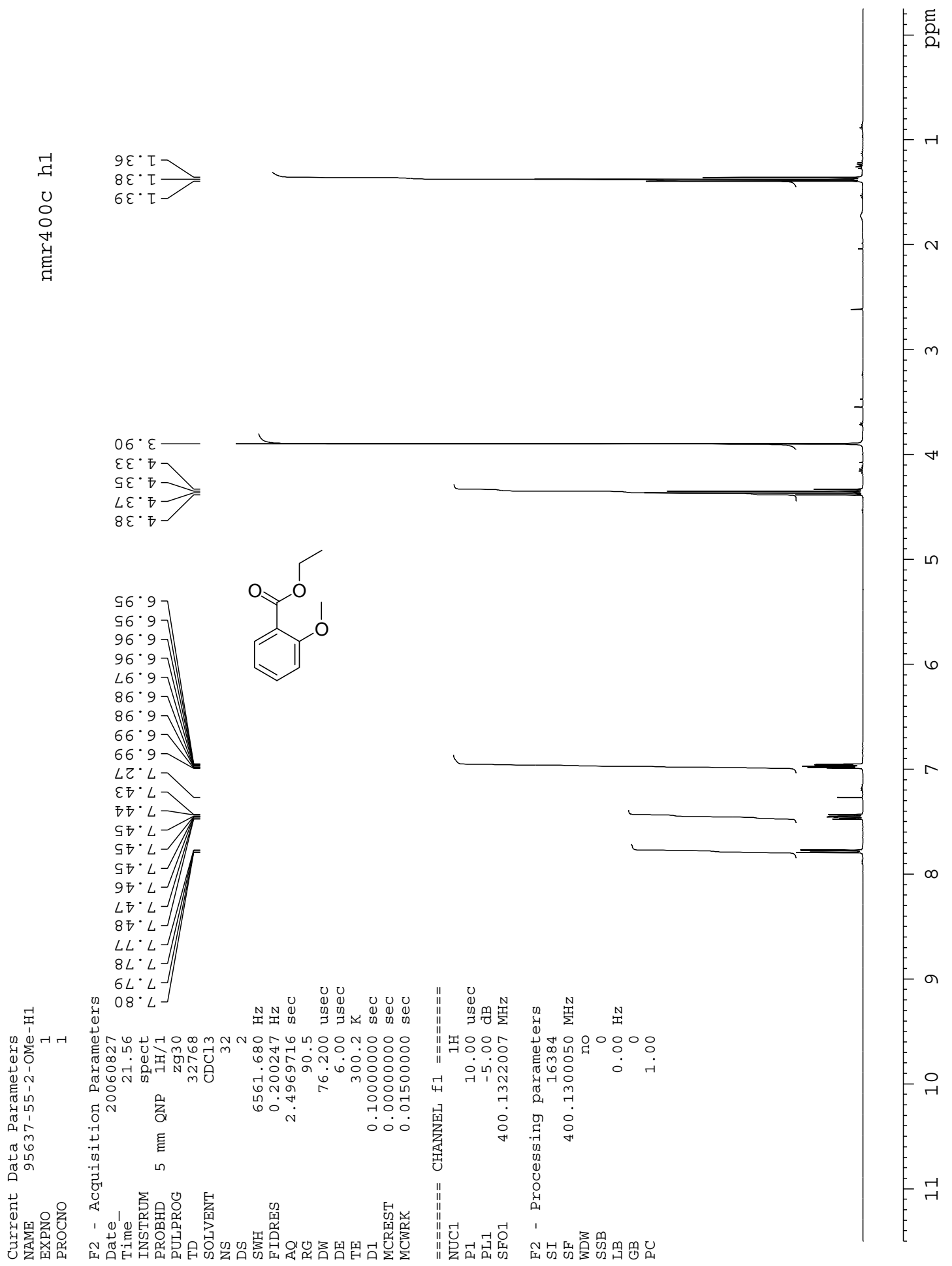


ethyl 2-cyanobenzoate (8)
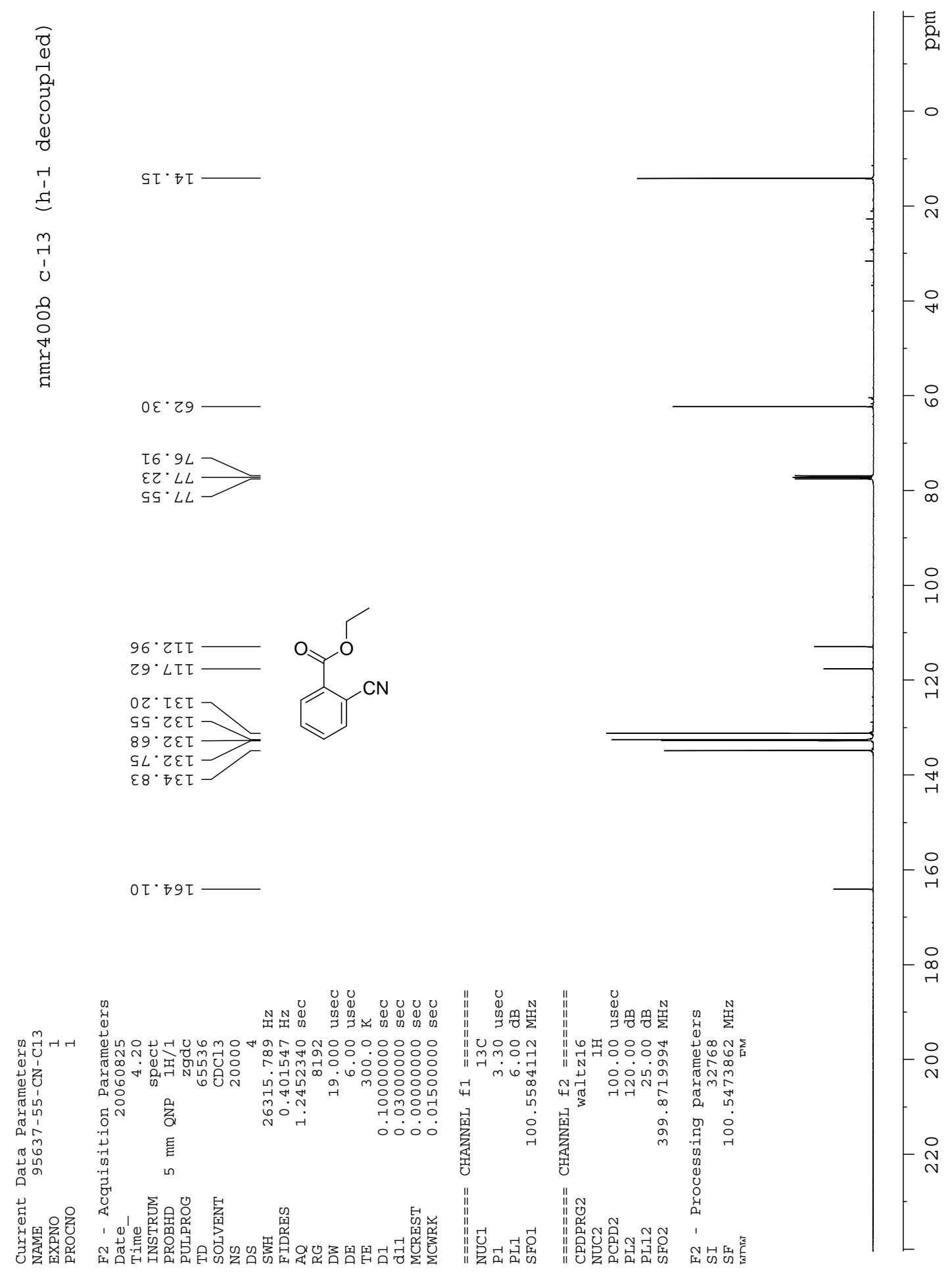
ethyl 2-cyanobenzoate (8)

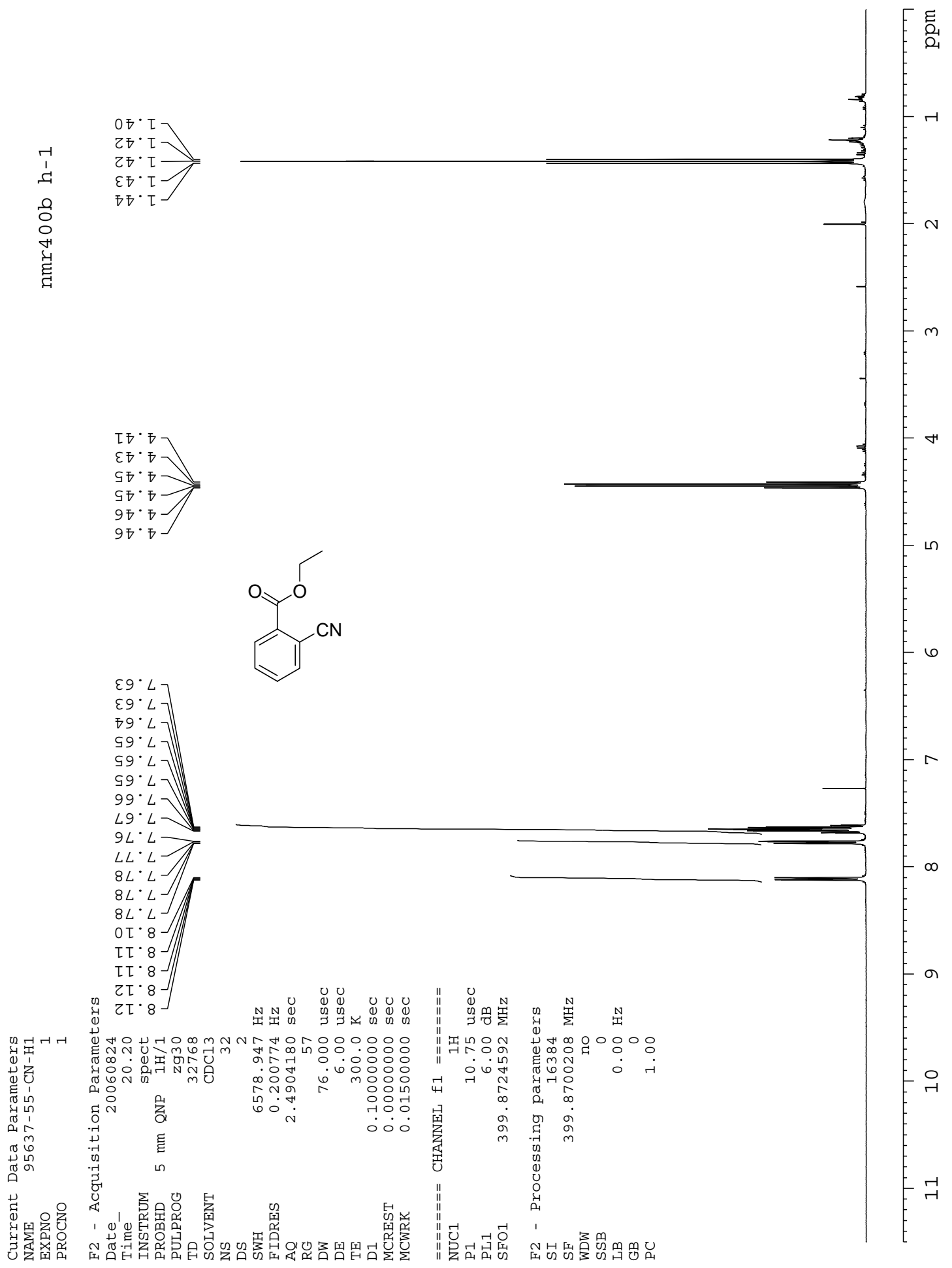


ethyl 4-chlorobenzoate (9)
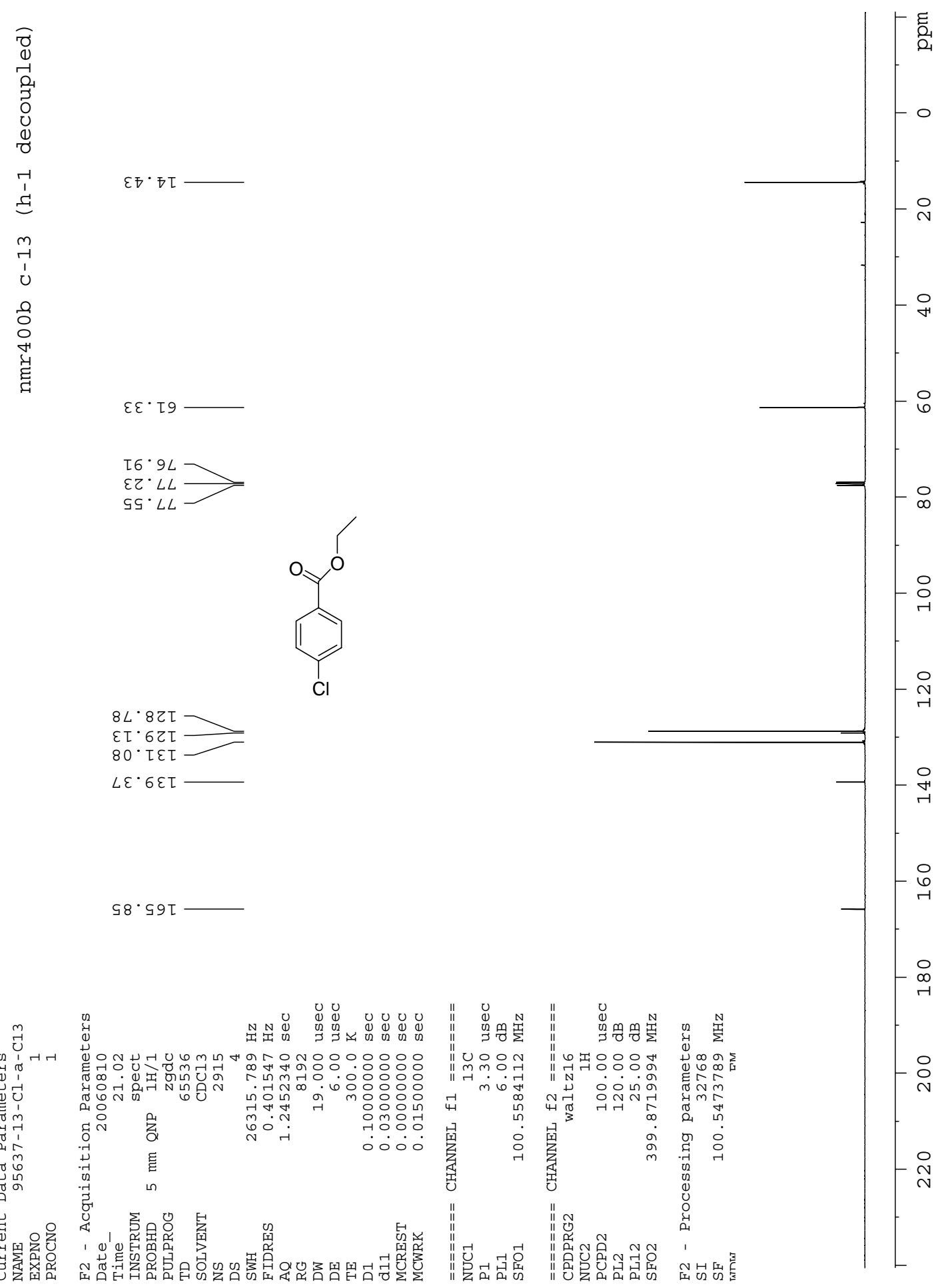
ethyl 4-chlorobenzoate (9)

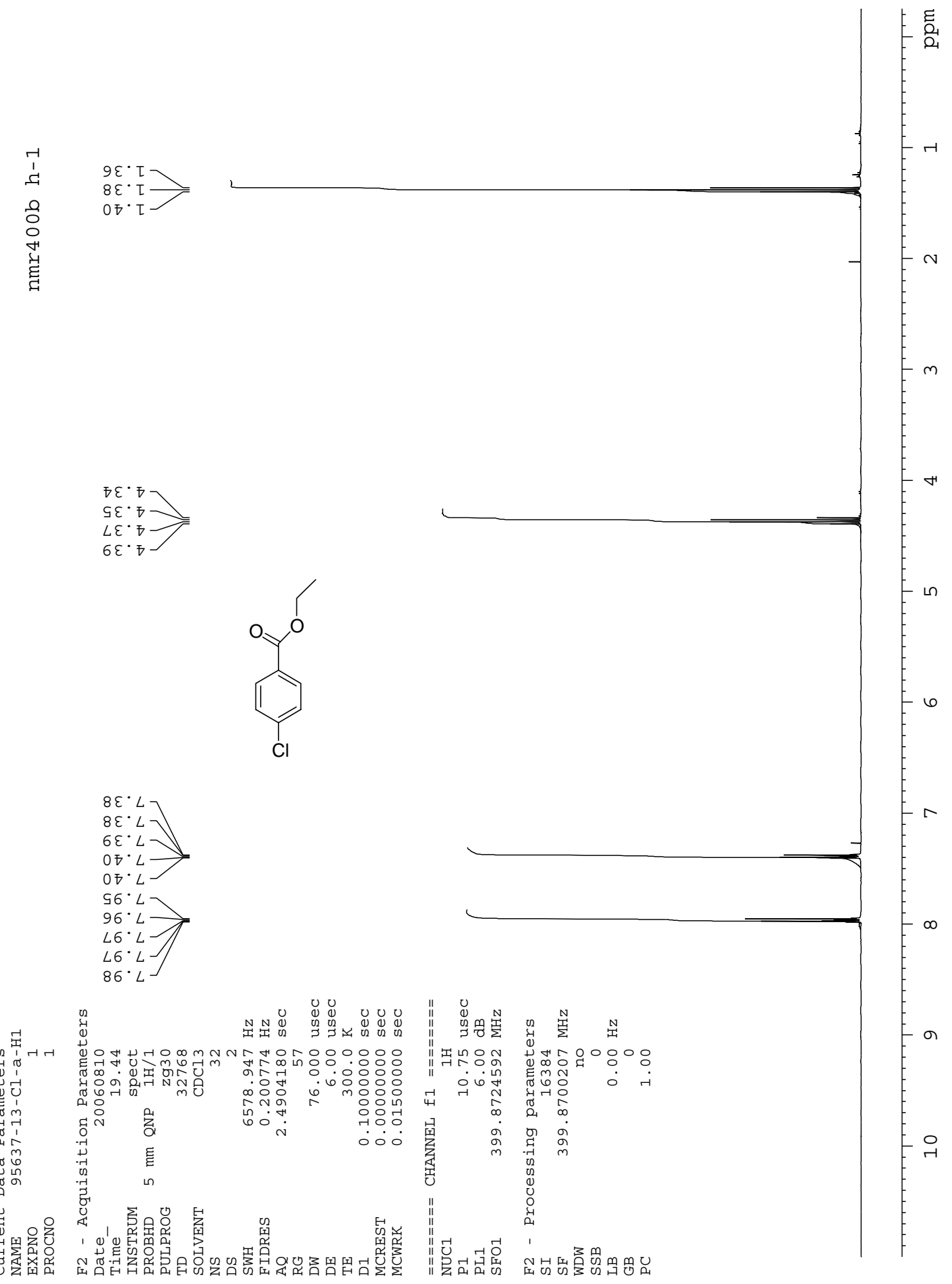


diethyl terephthalate (10)

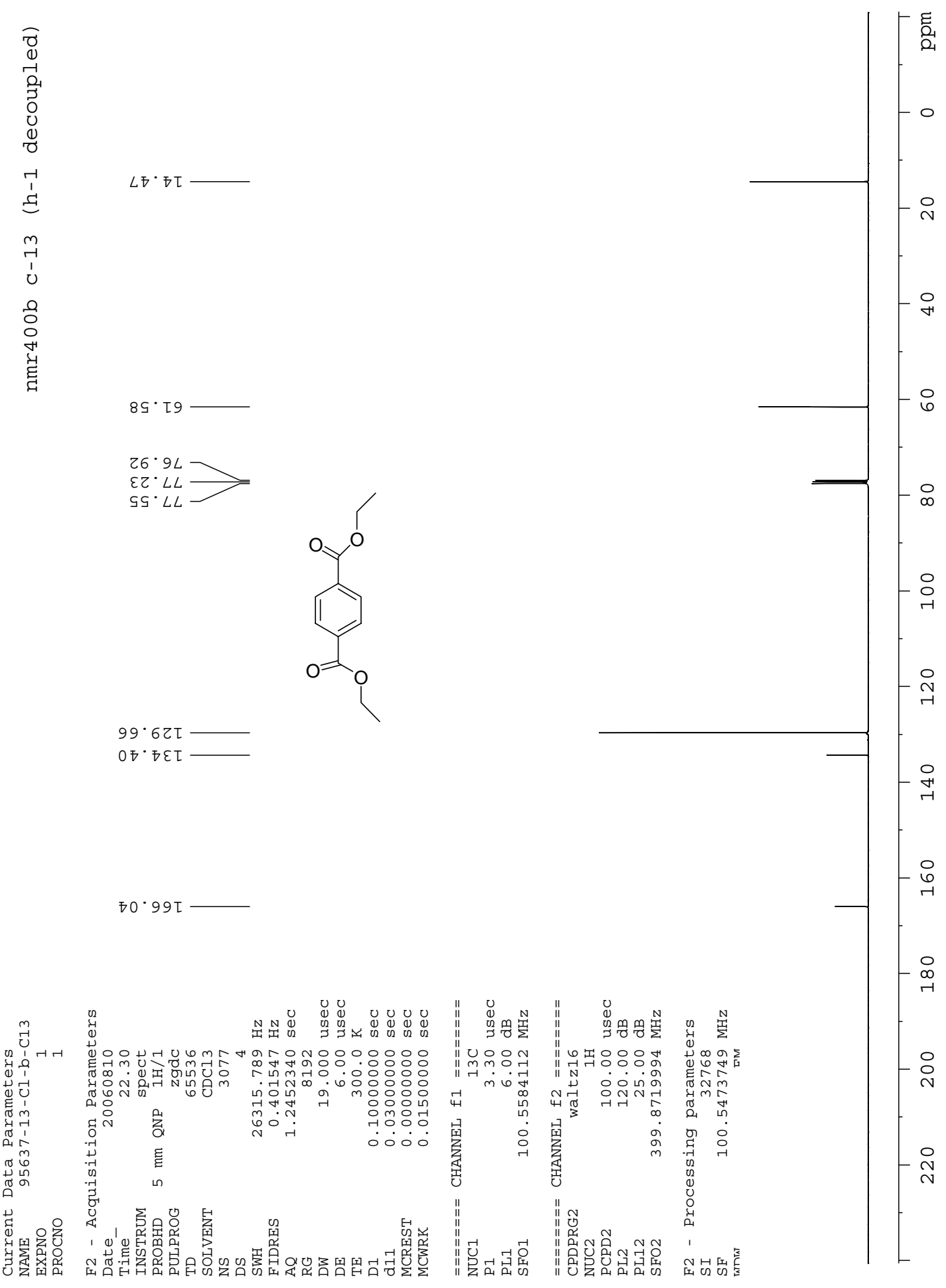




\section{diethyl terephthalate (10)}

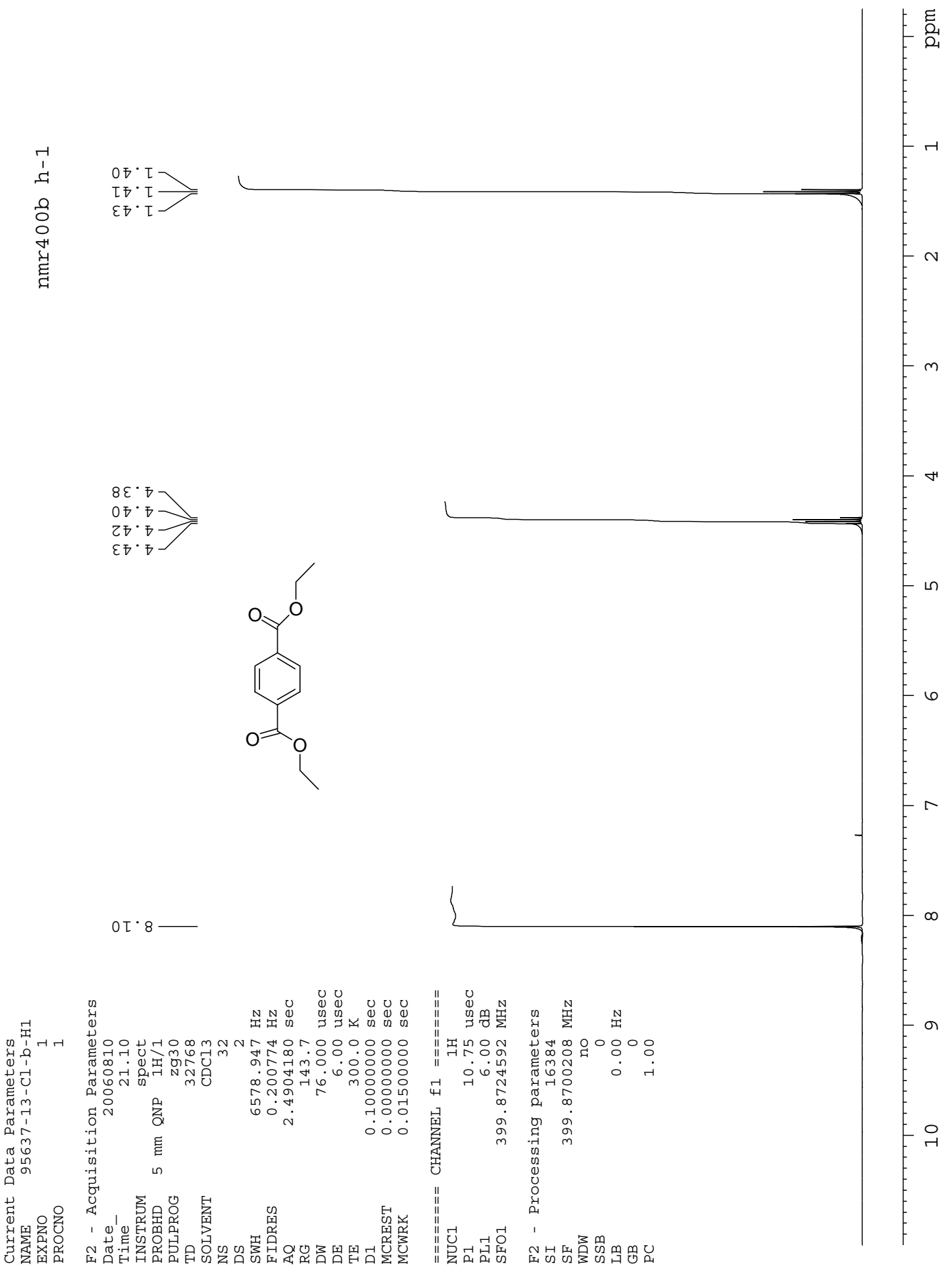


ethyl 4-fluorobenzoate (11)
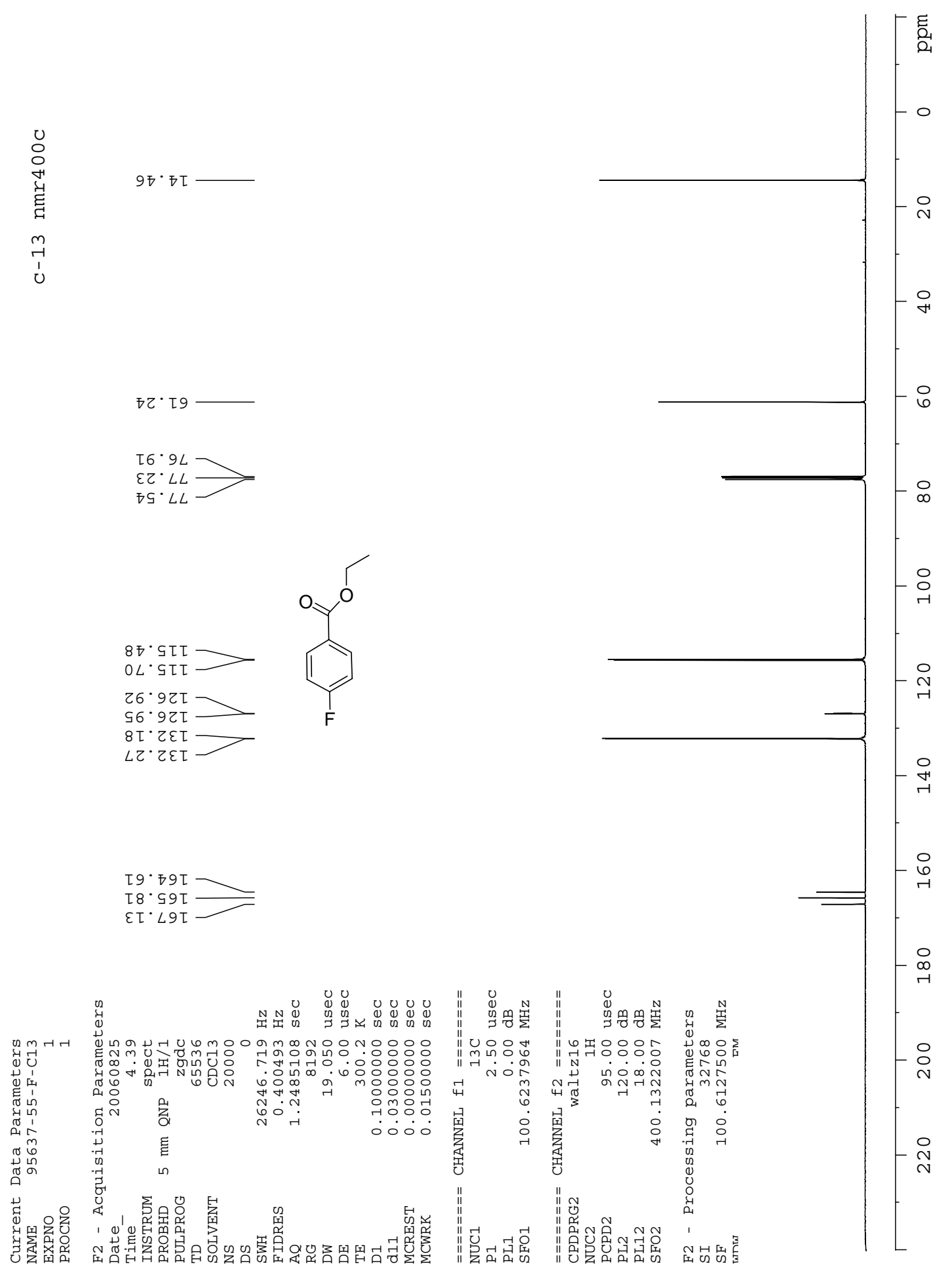


\section{ethyl 4-fluorobenzoate (11)}

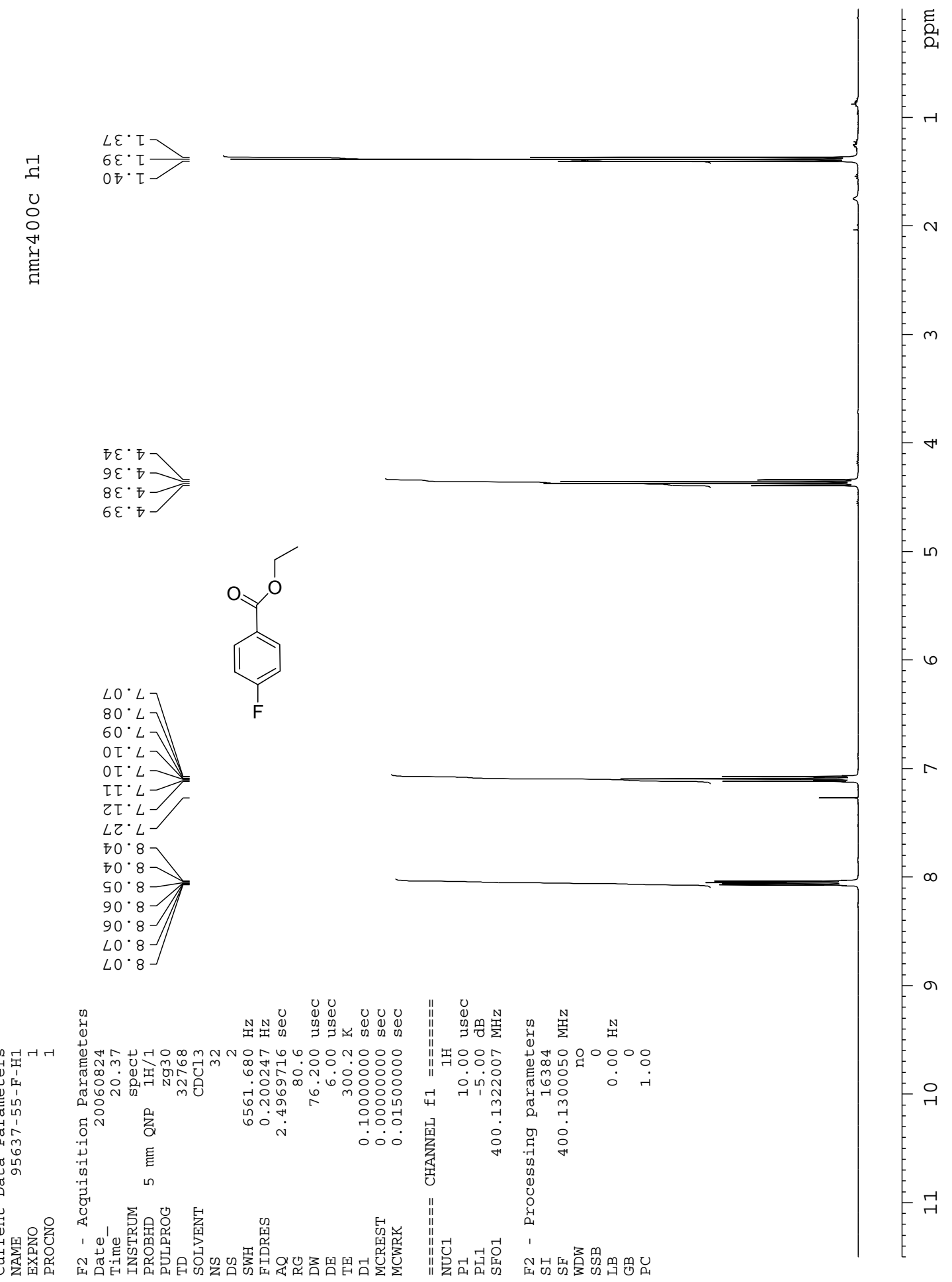


ethyl nicotinate (12)

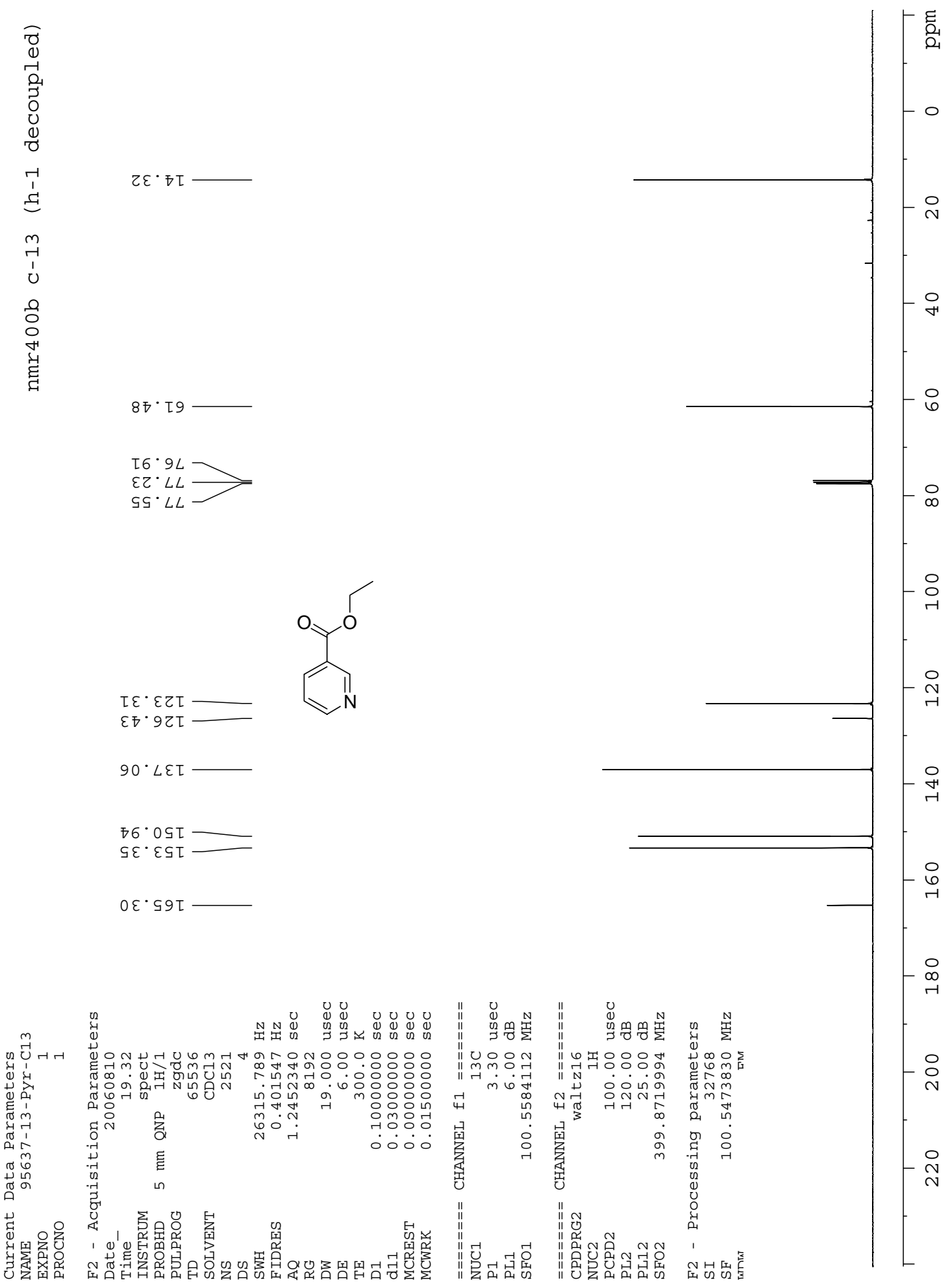




\section{ethyl nicotinate (12)}

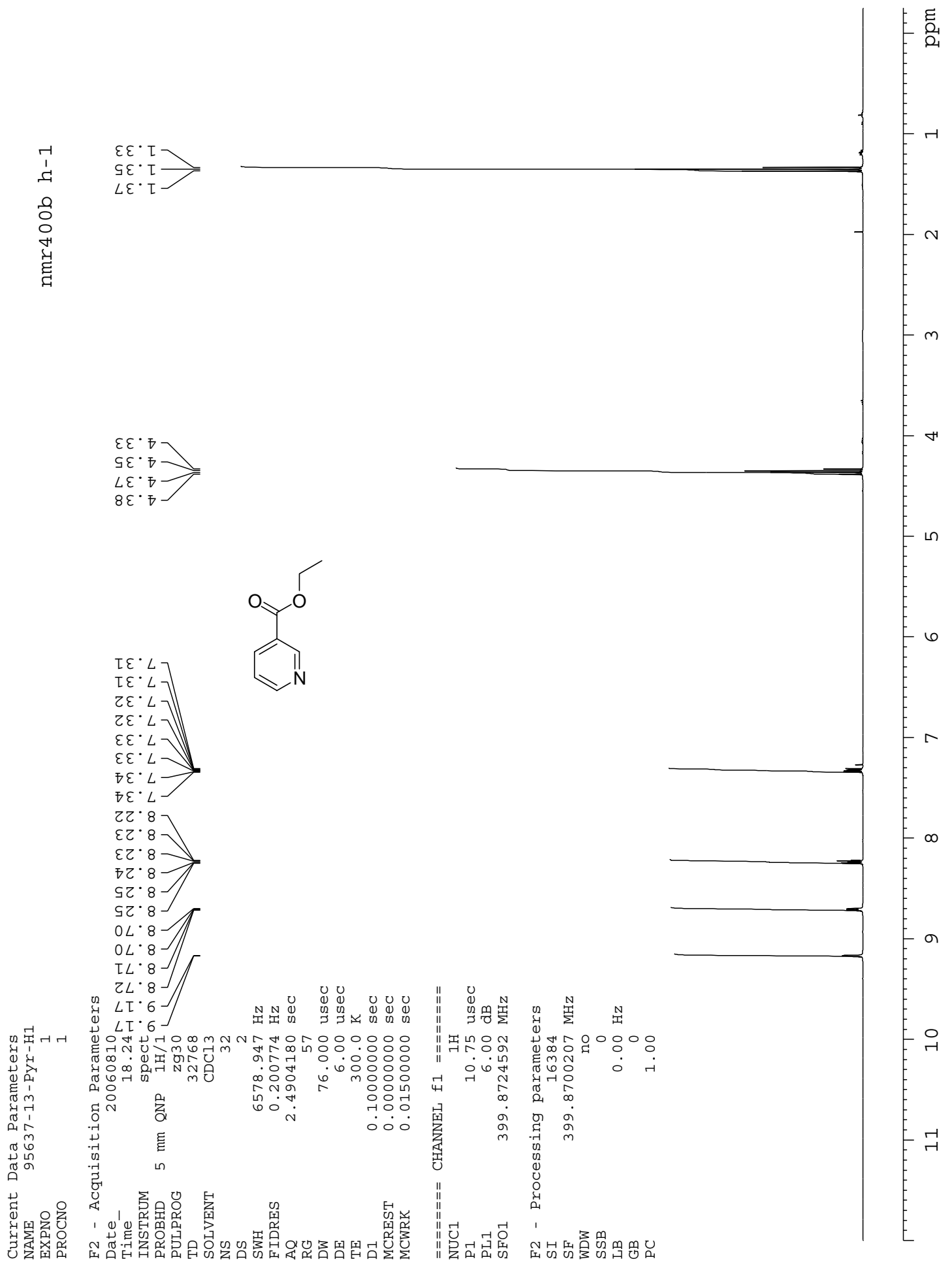


ethyl 4-cyanomethylbenzoate (13)
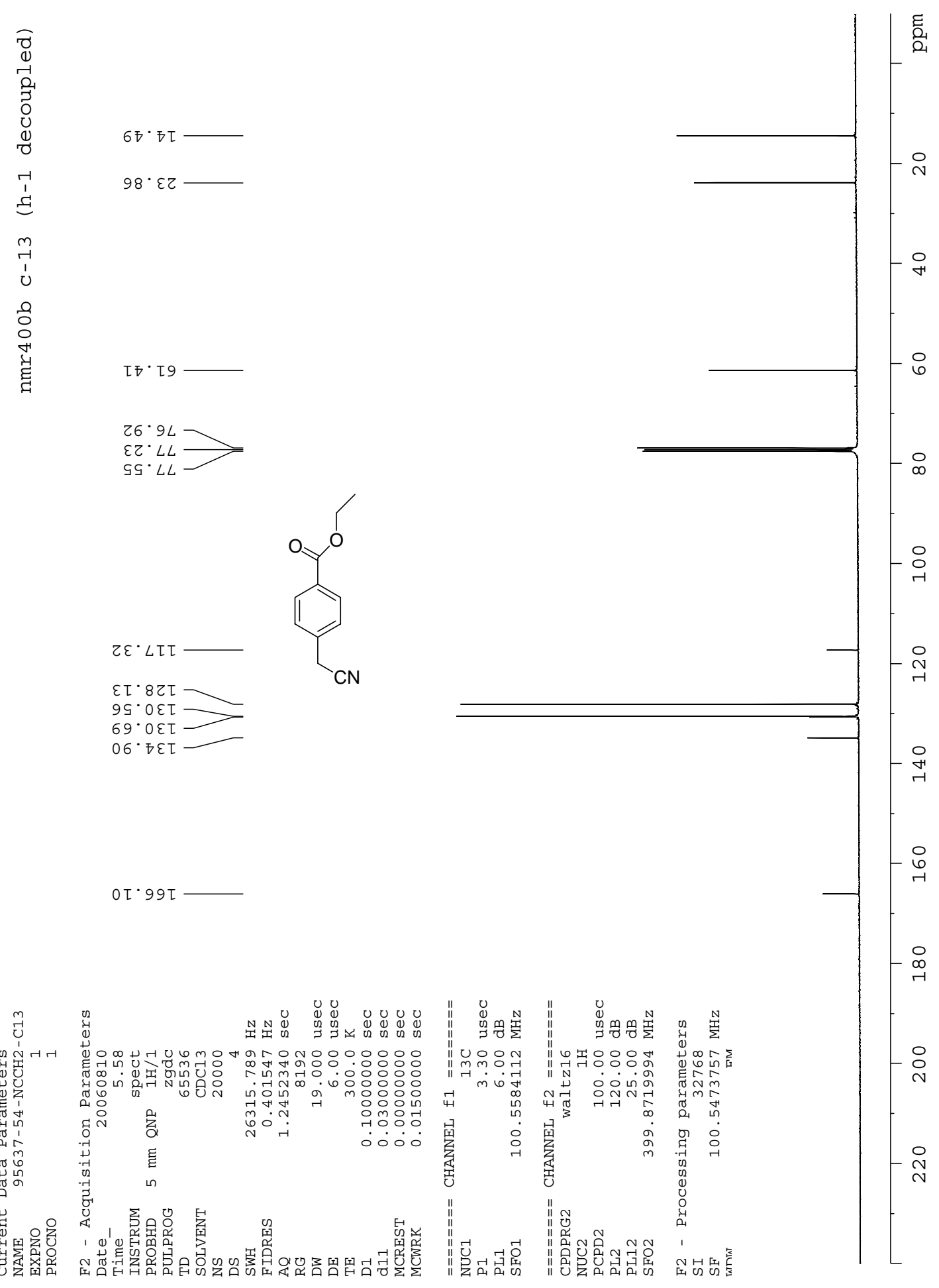
ethyl 4-cyanomethylbenzoate (13)

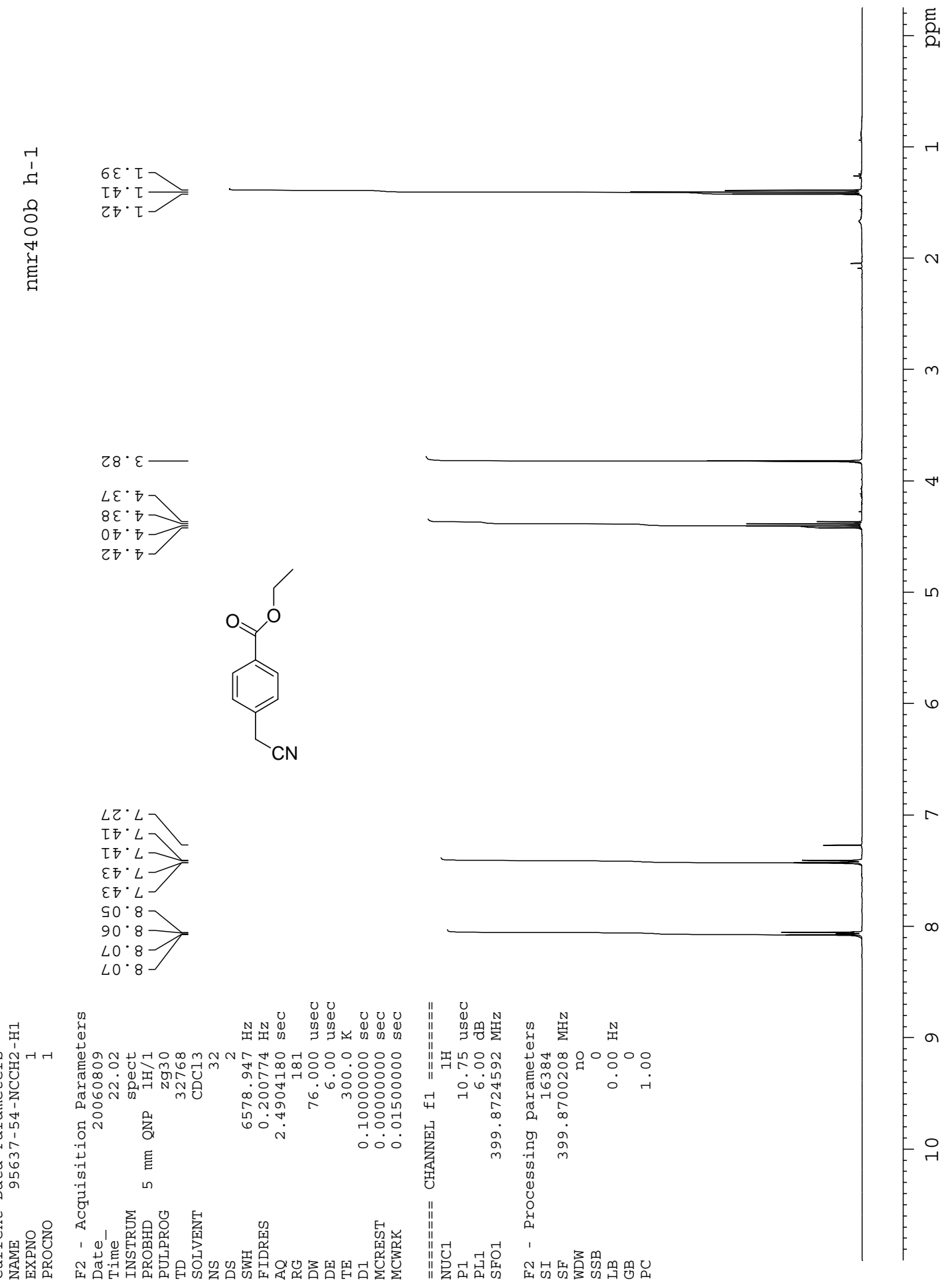




\section{4-acetylphenyl 4-fluorobenzenesulfonate (14)}
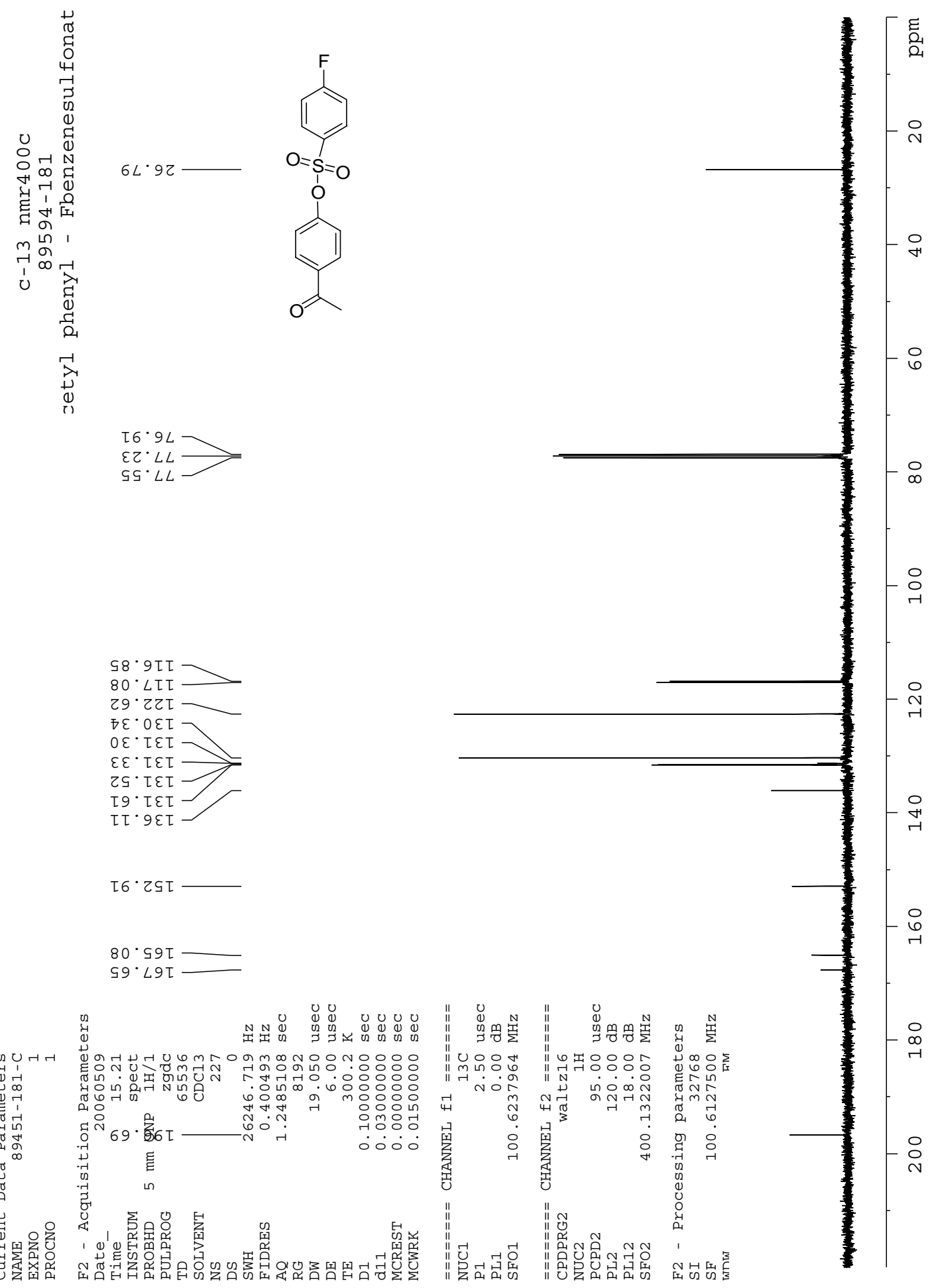


\section{4-acetylphenyl 4-fluorobenzenesulfonate (14)}

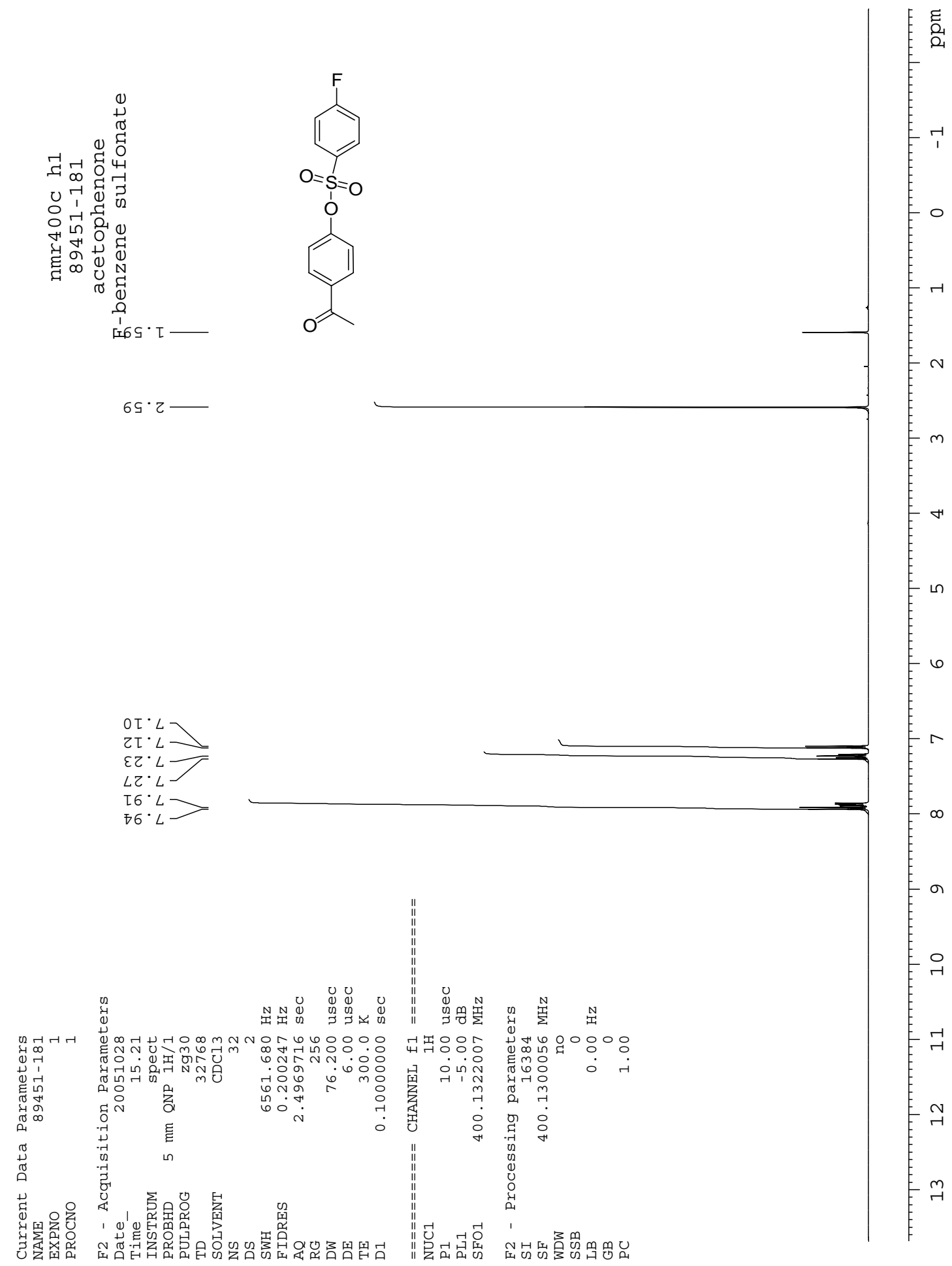




\section{phenyl 4-fluorobenzenesulfonate (15)}
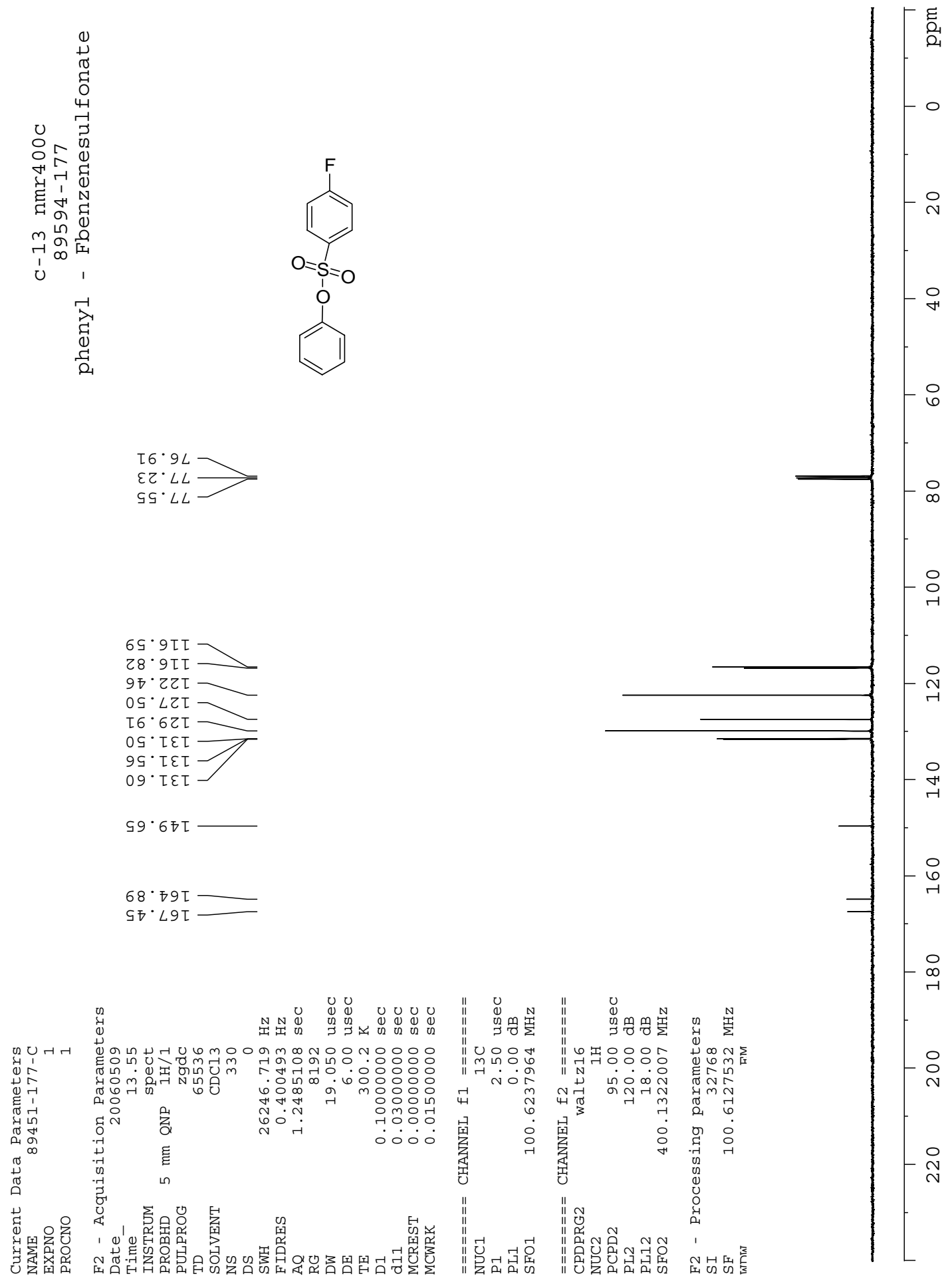


\section{phenyl 4-fluorobenzenesulfonate (15)}

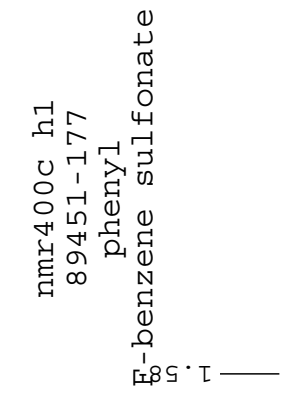<smiles>O=S(=O)(Oc1ccccc1)c1ccc(F)cc1</smiles>

王全

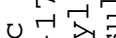

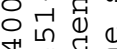

प्रति

도

$86 \cdot 97$

$66 \cdot 9$

66.

00.4

โ $0^{\circ} L$

$[0 \cdot L]$

$8 \mathrm{~T} \cdot \mathrm{L}$

$6 \tau \cdot L]$

$0 乙 \cdot L]$

$z z \cdot L$

$\varepsilon 乙 \cdot L]$

$L Z \cdot L]$

$L \tau \cdot L]$

$8 z^{\circ}$

$8 z \cdot L$

$62 \cdot L$

$6 \tau \cdot L J$

$[\varepsilon \cdot L]$

$[\varepsilon \cdot L]$

$\tau \varepsilon \cdot L$

$\tau \varepsilon \cdot L$

$\varepsilon \varepsilon \cdot L$

$\varepsilon \varepsilon \cdot L-$

\rceil$\varepsilon \cdot L]$

$\mp 8 \cdot L$

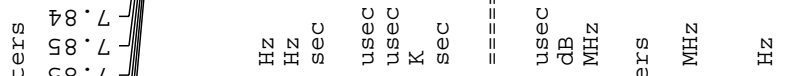




\section{4-methylphenyl 4-fluorobenzenesulfonate (16)}

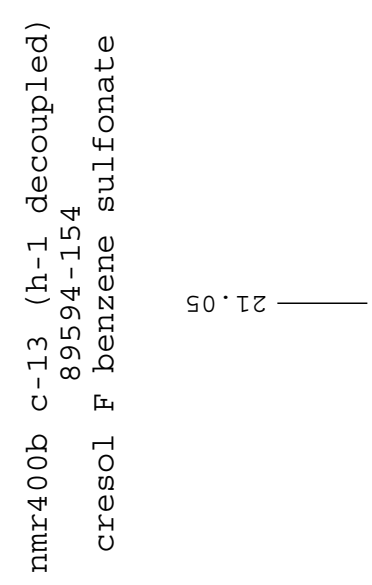<smiles>Cc1ccc(OS(=O)(=O)c2ccc(F)cc2)cc1</smiles>
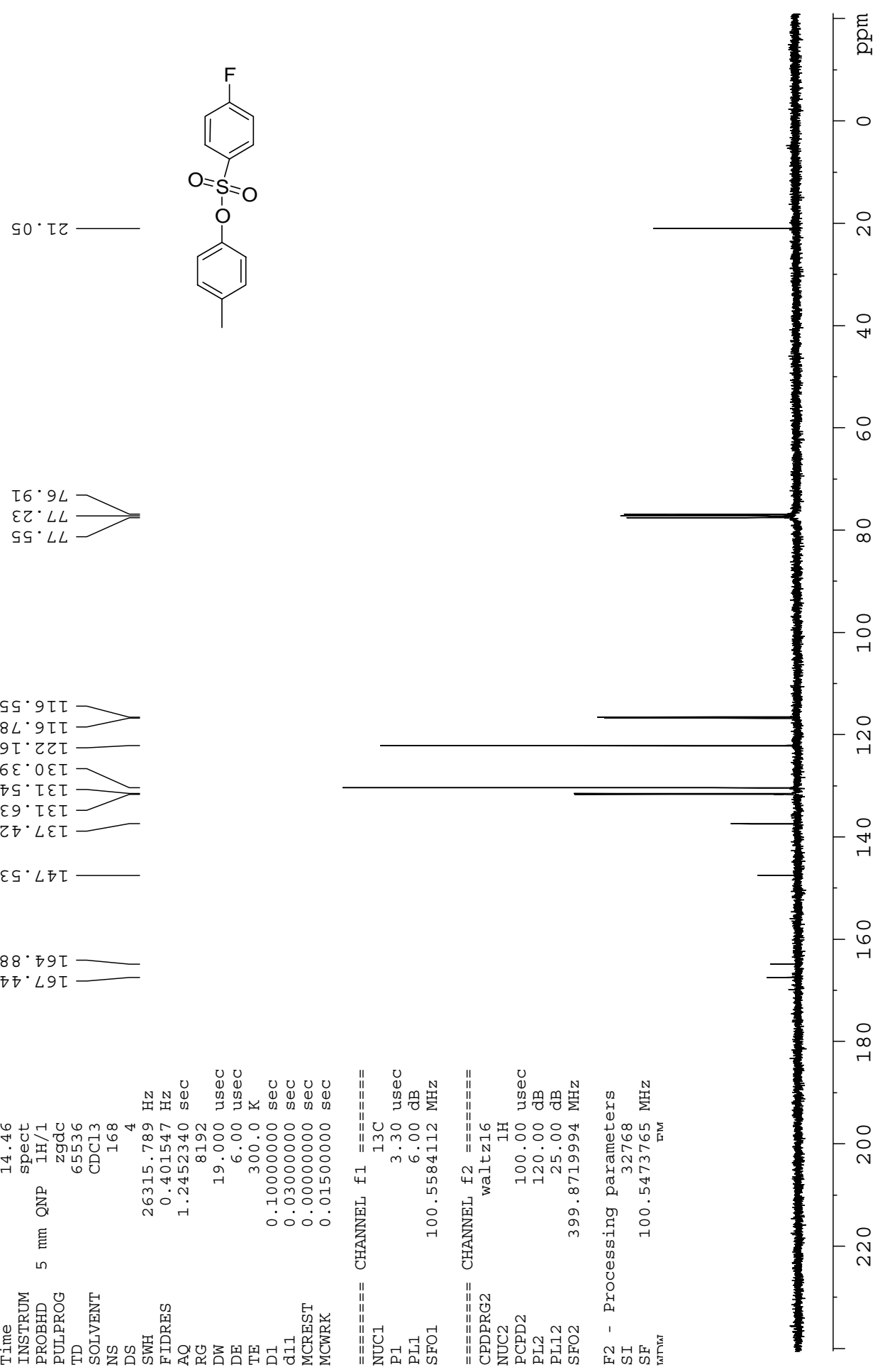


\section{4-methylphenyl 4-fluorobenzenesulfonate (16)}

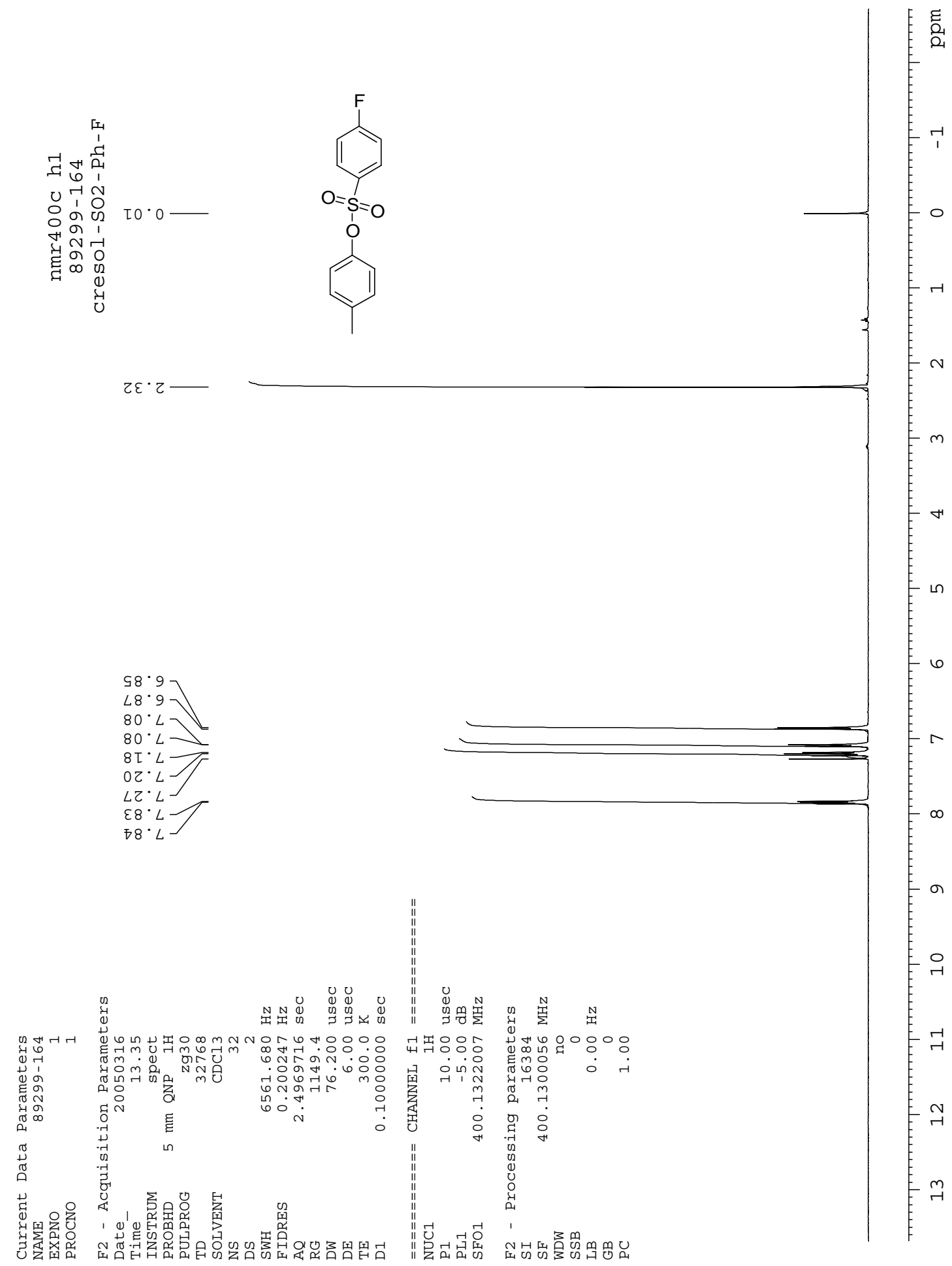




\section{2-methylphenyl 4-fluorobenzenesulfonate (17)}
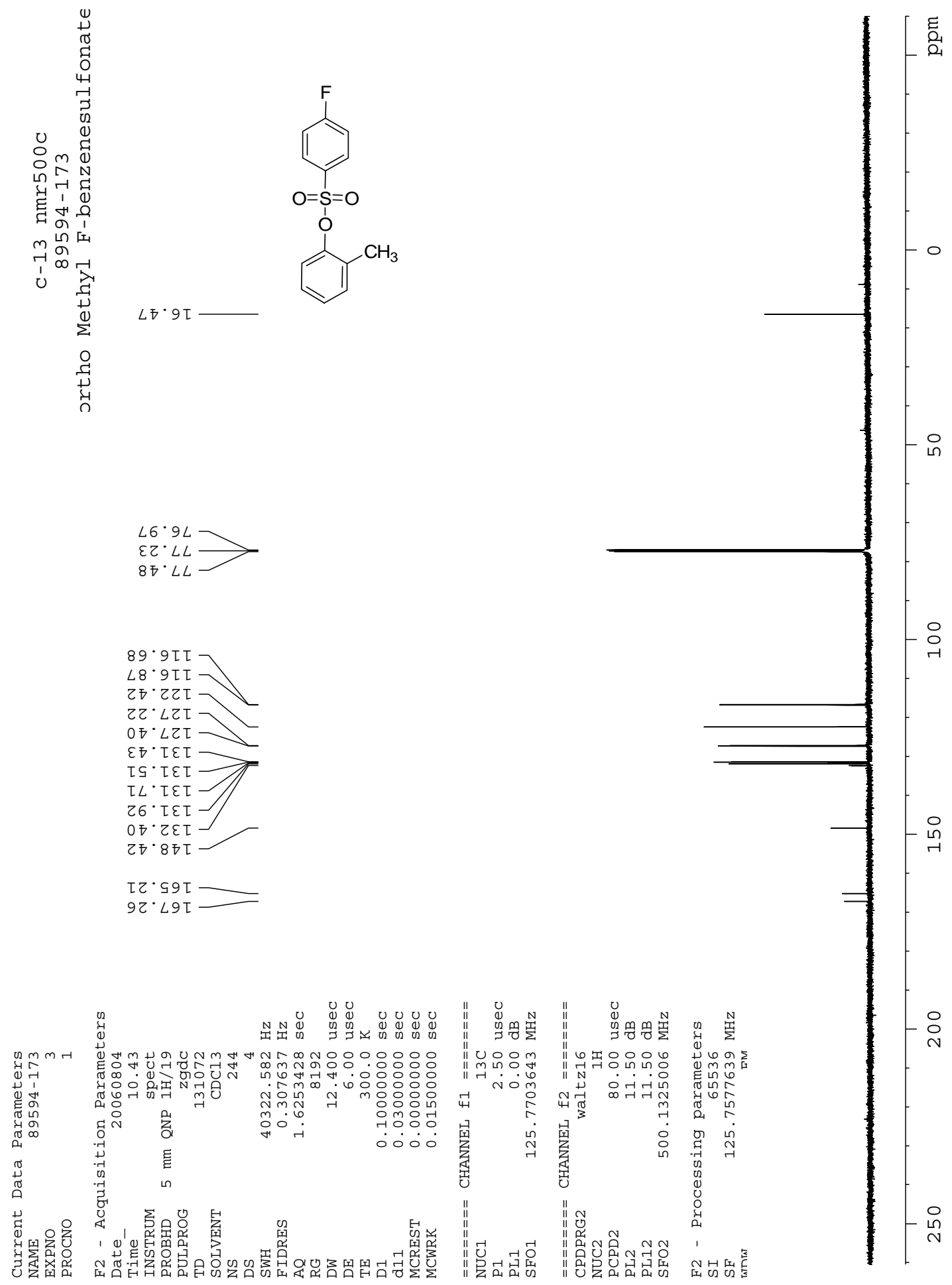


\section{2-methylphenyl 4-fluorobenzenesulfonate (17)}

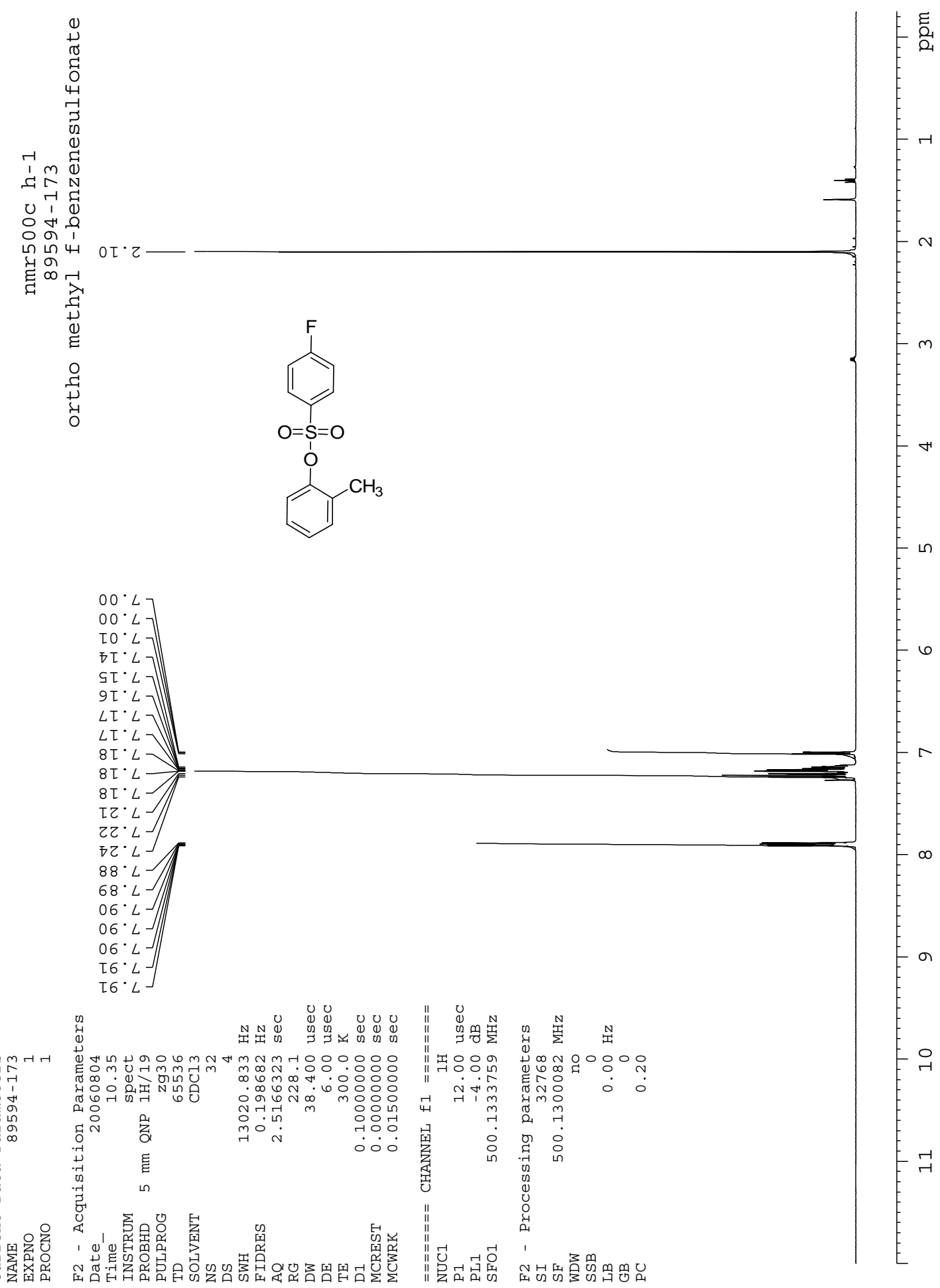




\section{4-methoxyphenyl 4-fluorobenzenesulfonate (18)}
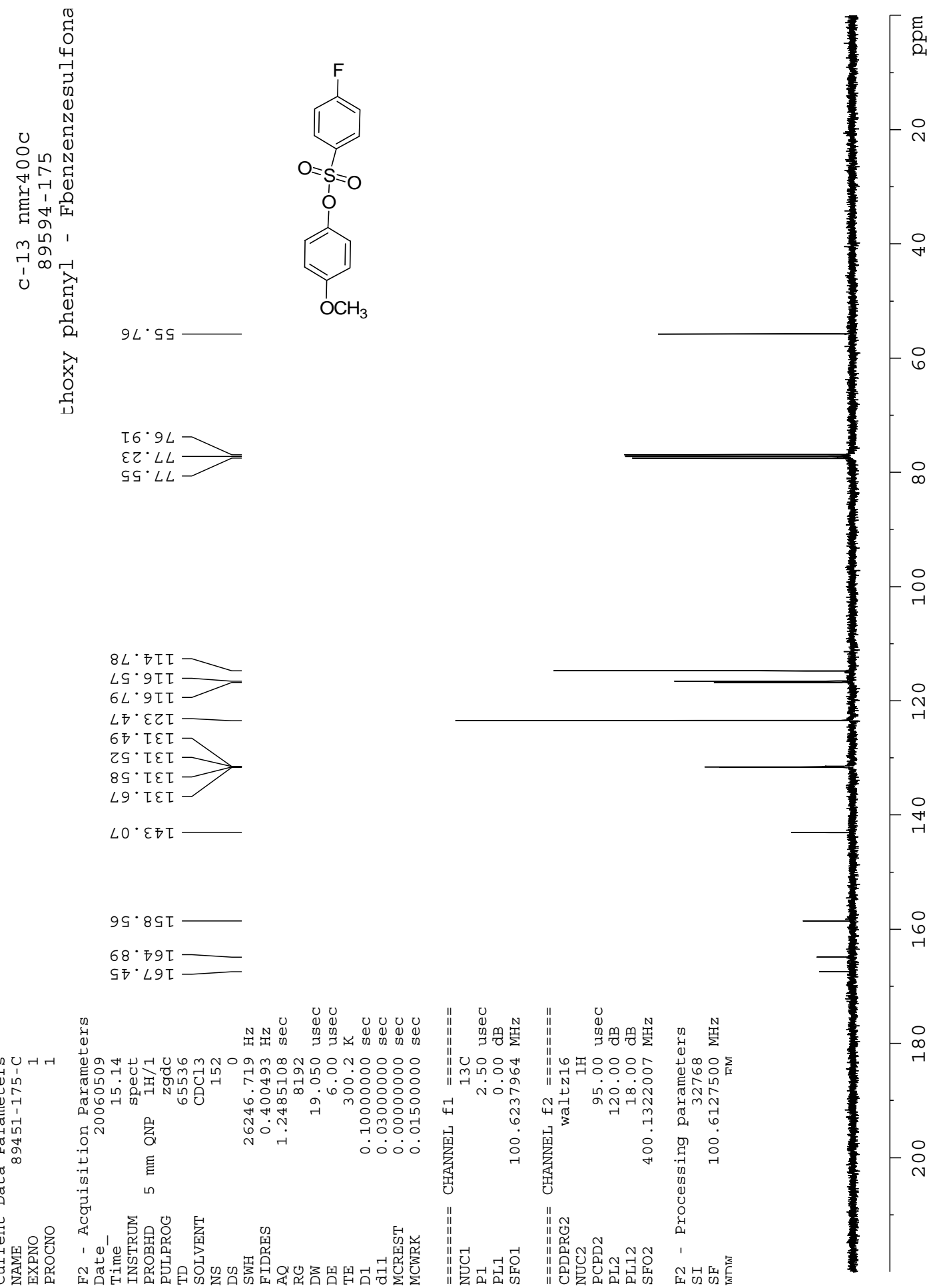


\section{4-methoxyphenyl 4-fluorobenzenesulfonate (18)}

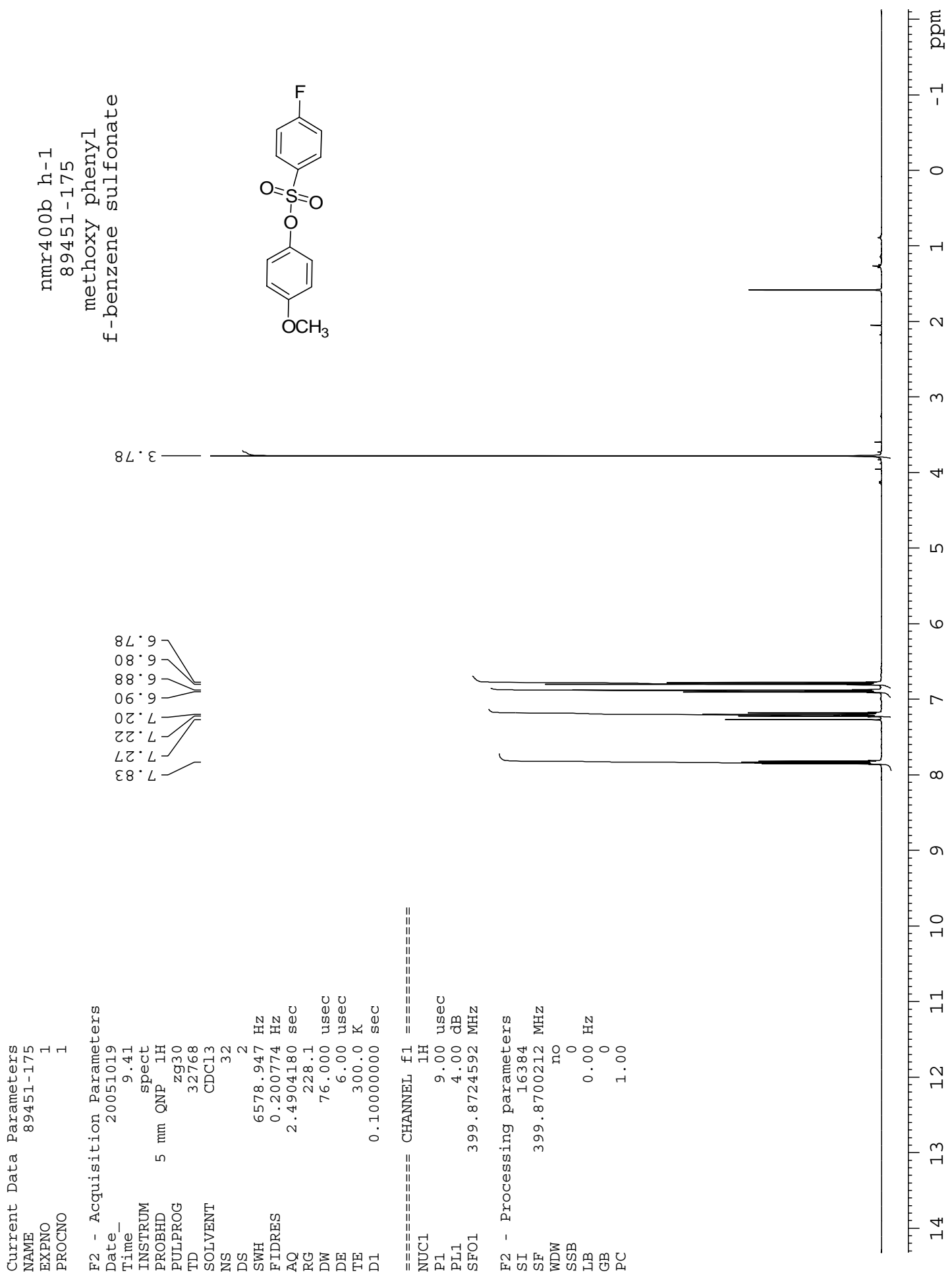




\section{2-methoxyphenyl 4-fluorobenzenesulfonate (19)}
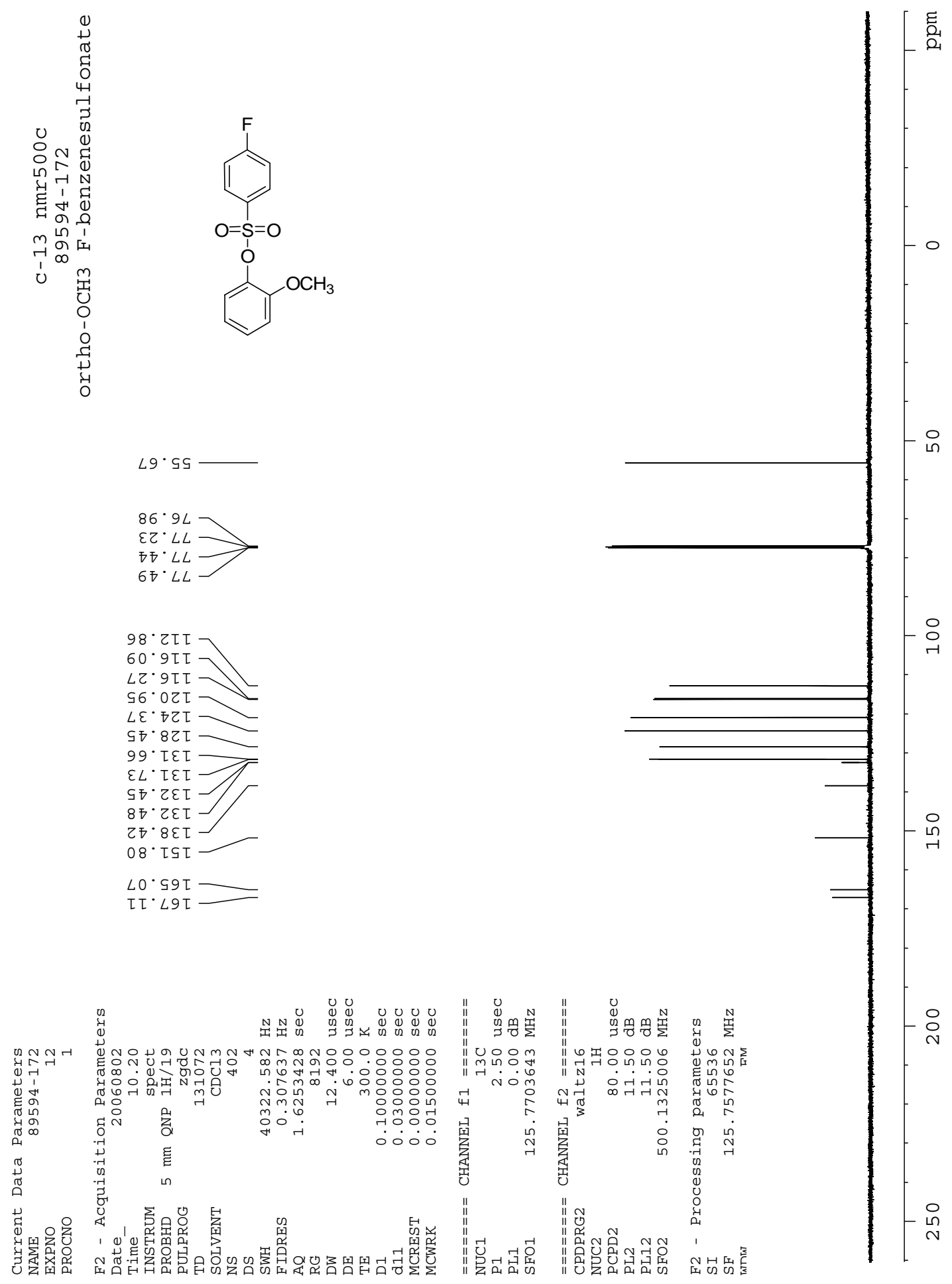


\section{2-methoxyphenyl 4-fluorobenzenesulfonate (19)}

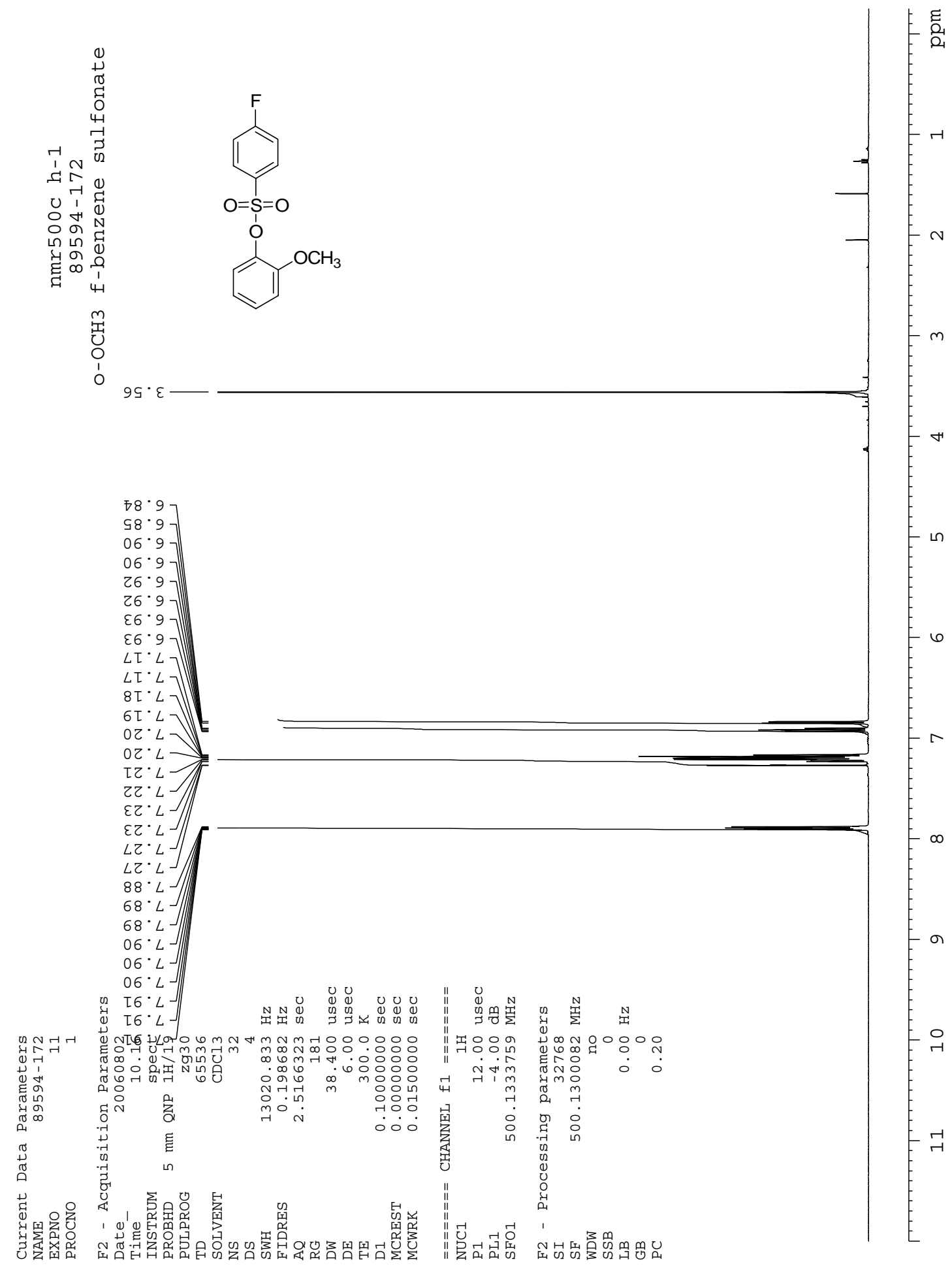




\section{2-cyanophenyl 4-fluorobenzenesulfonate (20)}
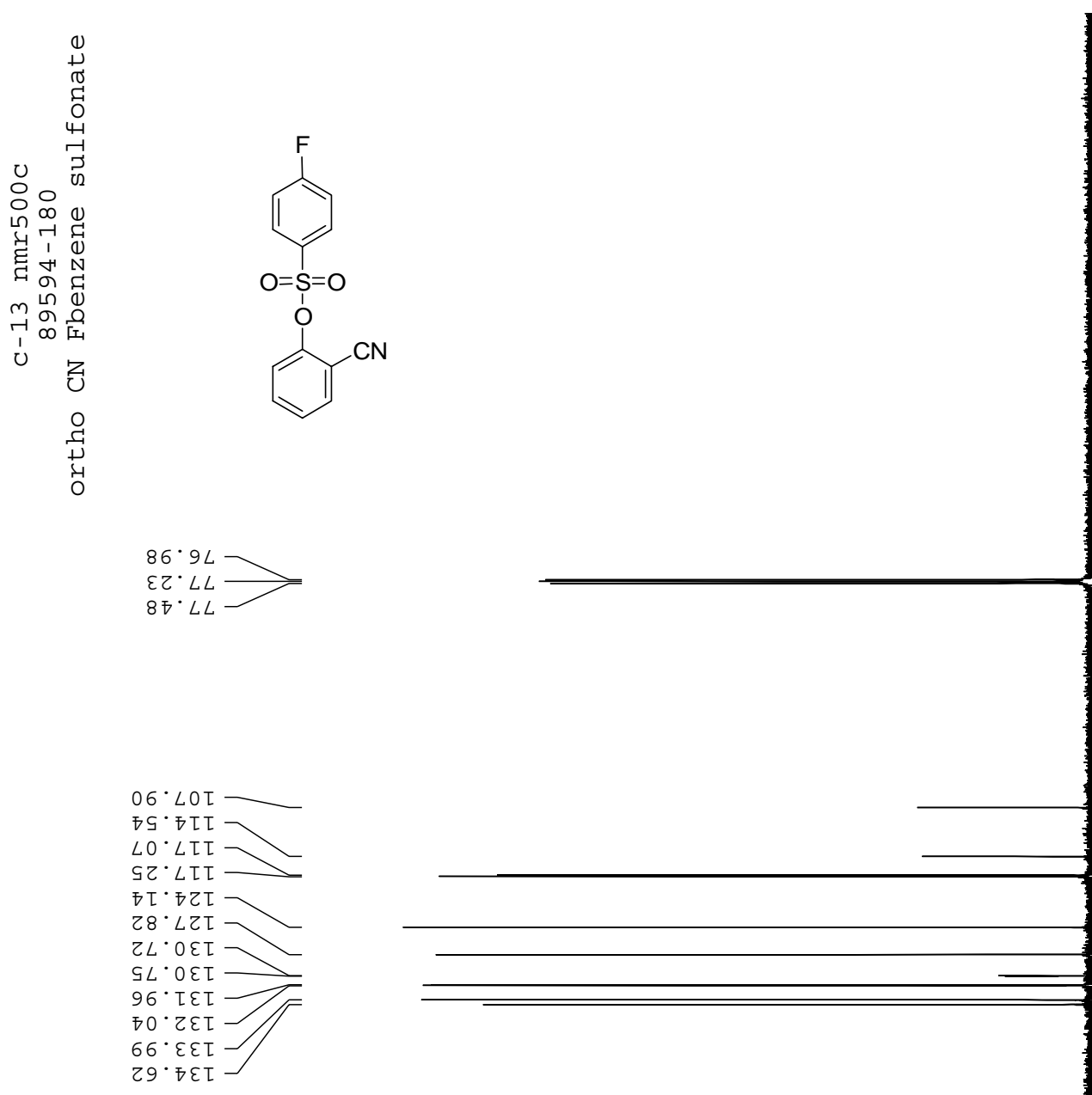

$\tau 乙 \cdot 0 \subseteq \tau$

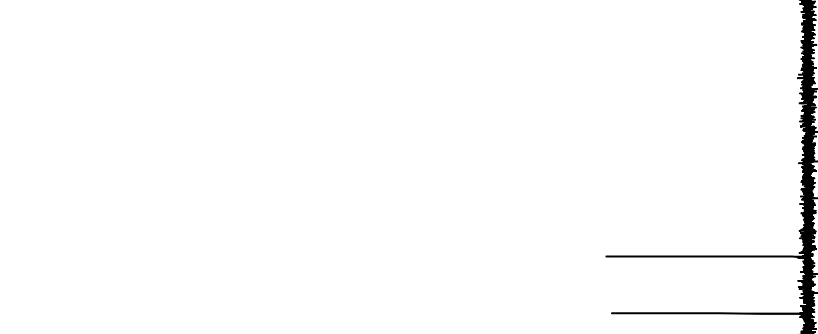

$+\infty$

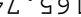

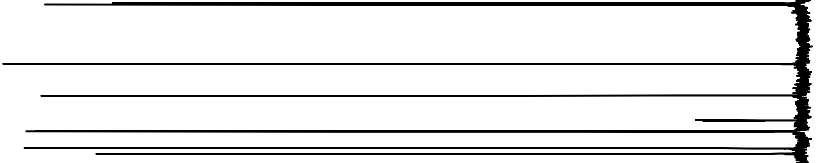

$08 \cdot 49 T$
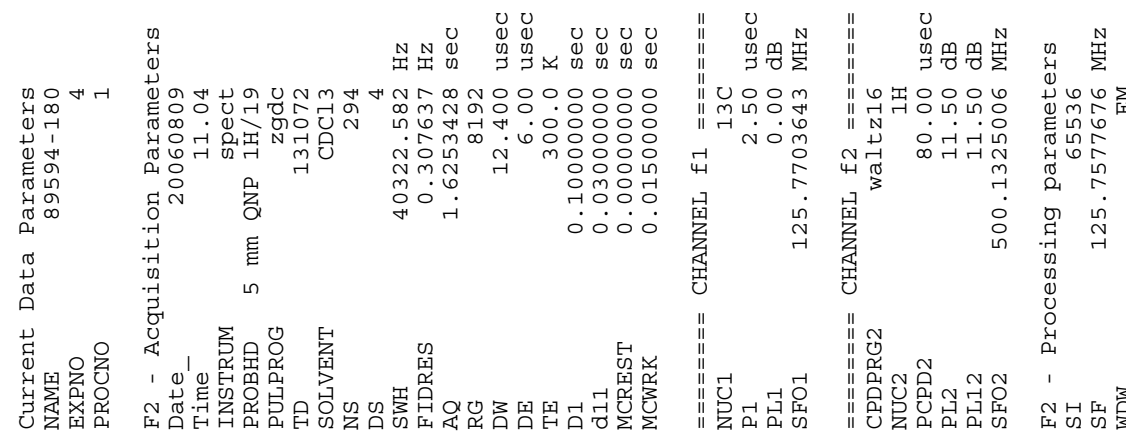

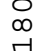

(1)

$\stackrel{\circ}{\circ}$ 


\section{2-cyanophenyl 4-fluorobenzenesulfonate (20)}

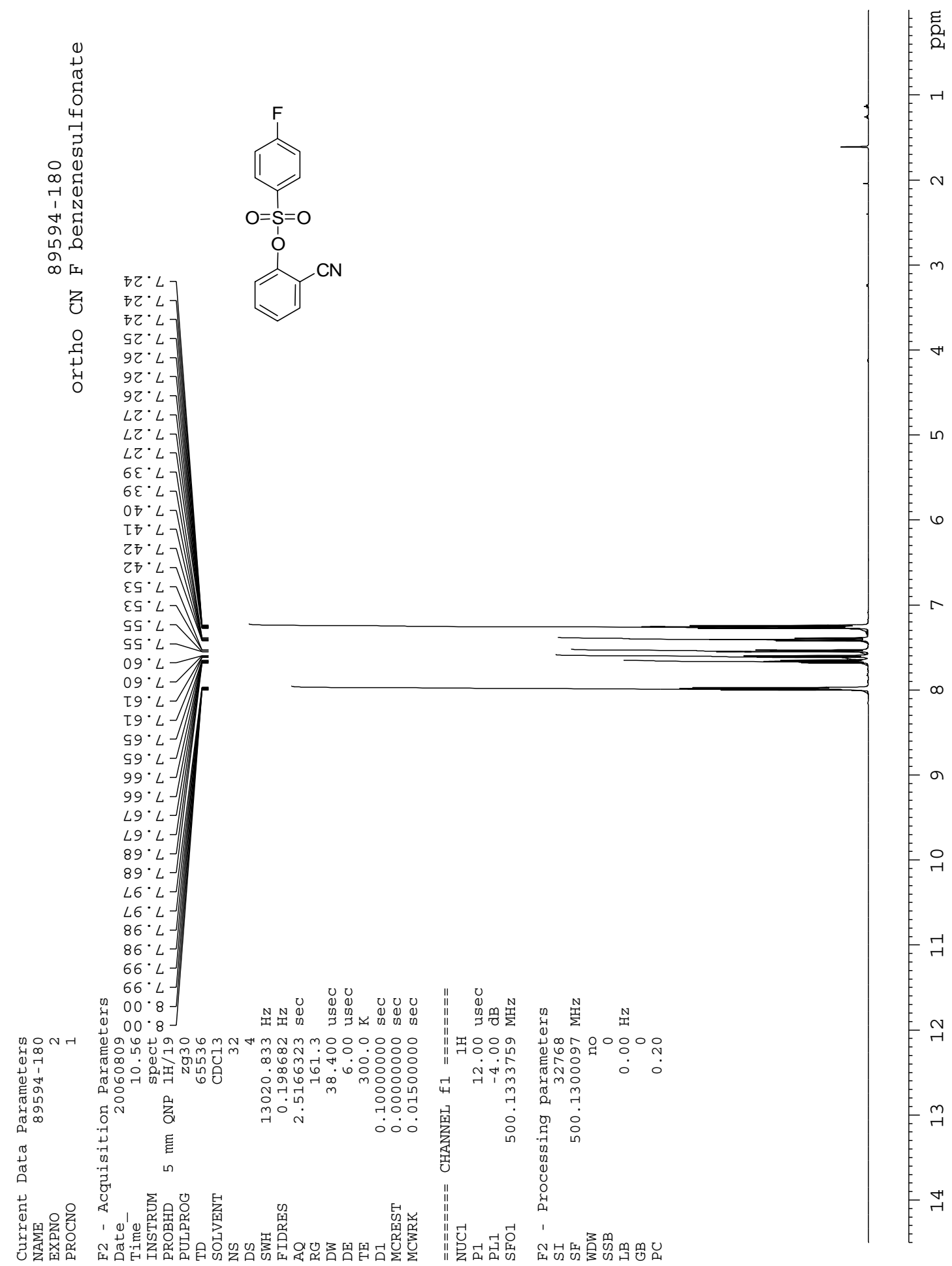




\section{4-chlorophenyl 4-fluorobenzenesulfonate (21)}

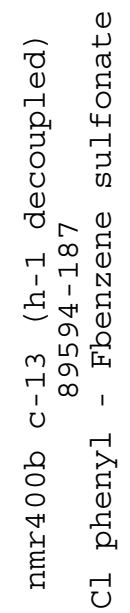<smiles>O=S(=O)(Oc1ccc(Cl)cc1)c1ccc(F)cc1</smiles>
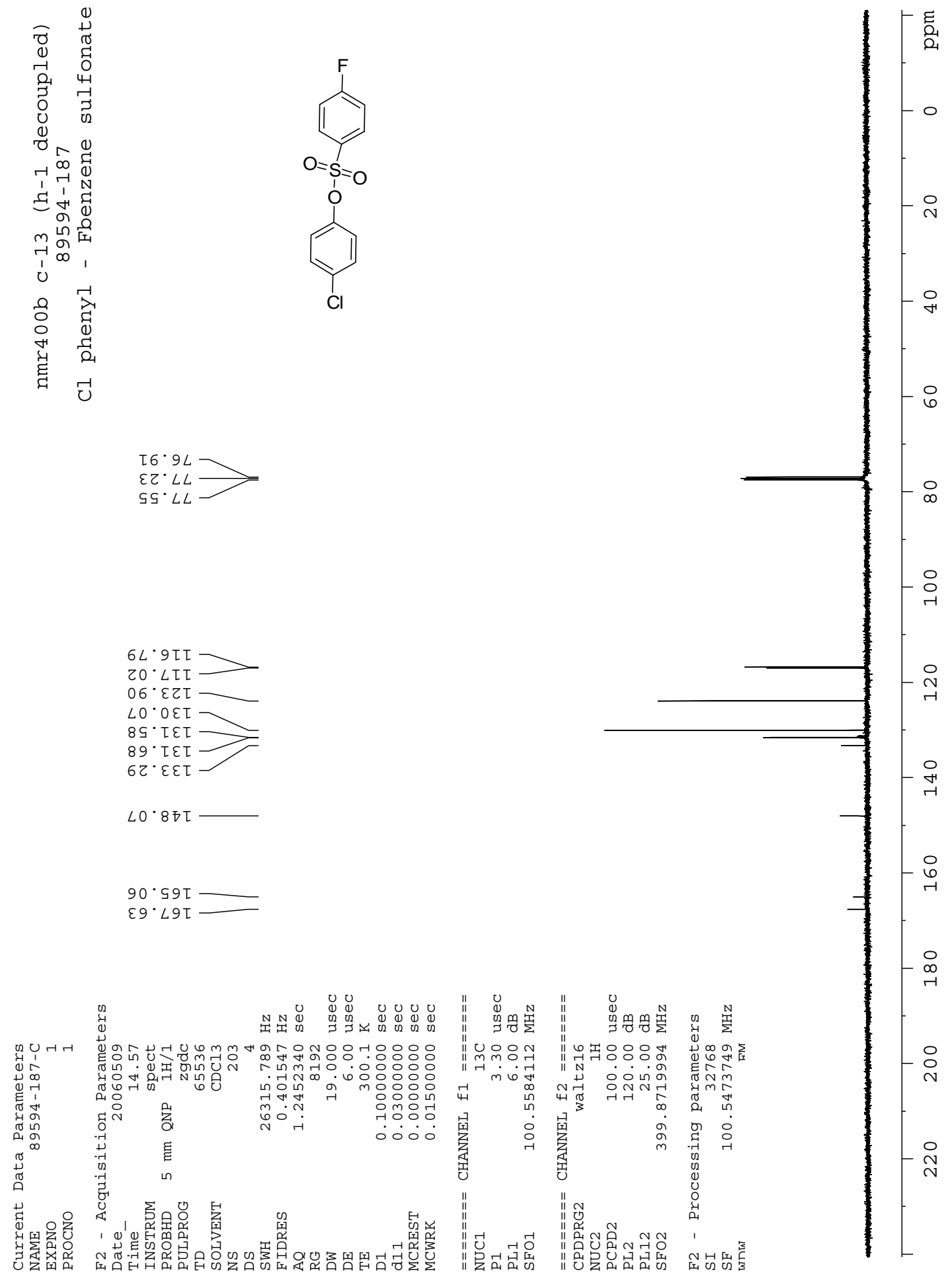


\section{4-chlorophenyl 4-fluorobenzenesulfonate (21)}
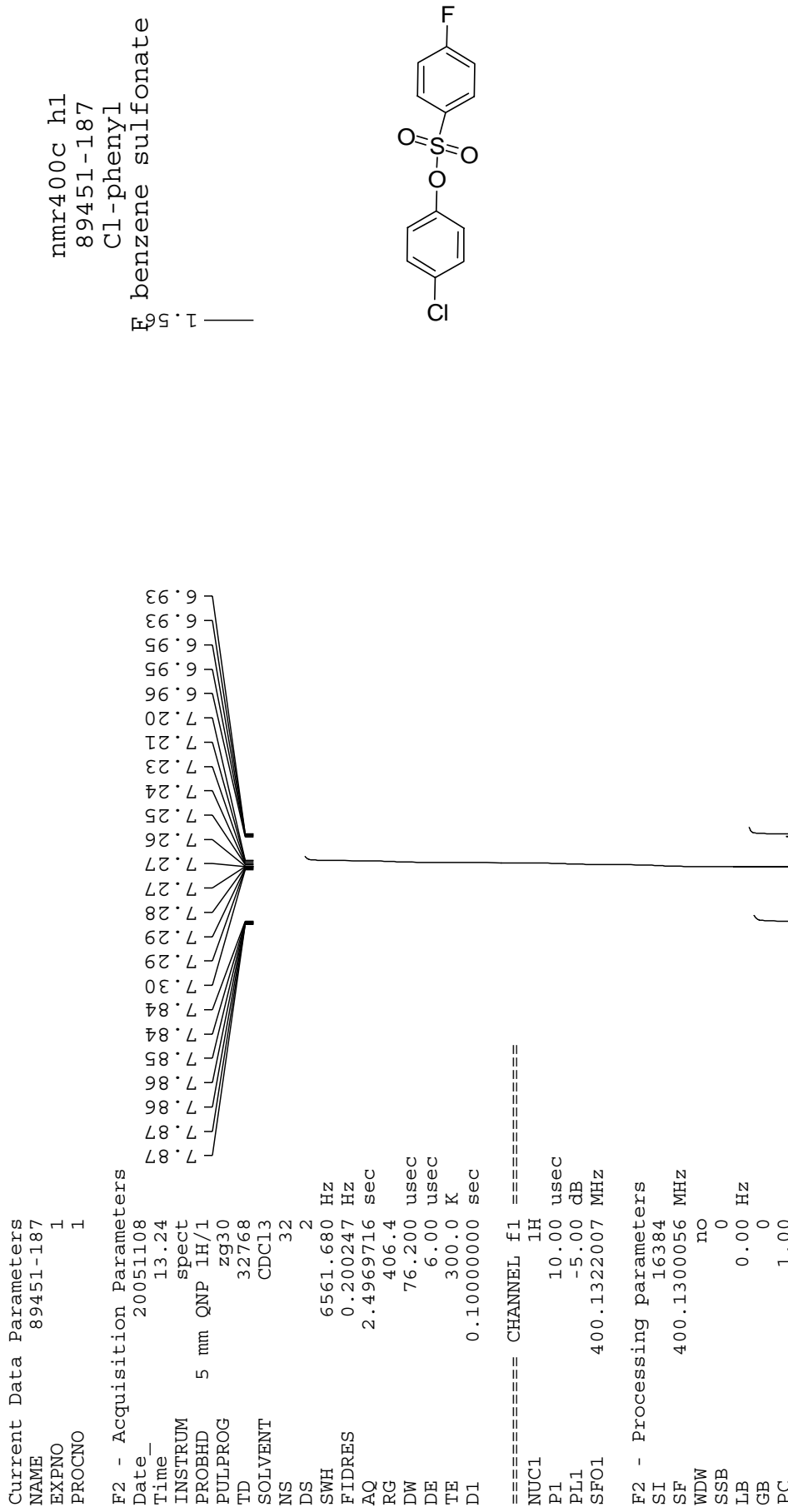


\section{4-flurophenyl 4-fluorobenzenesulfonate (22)}
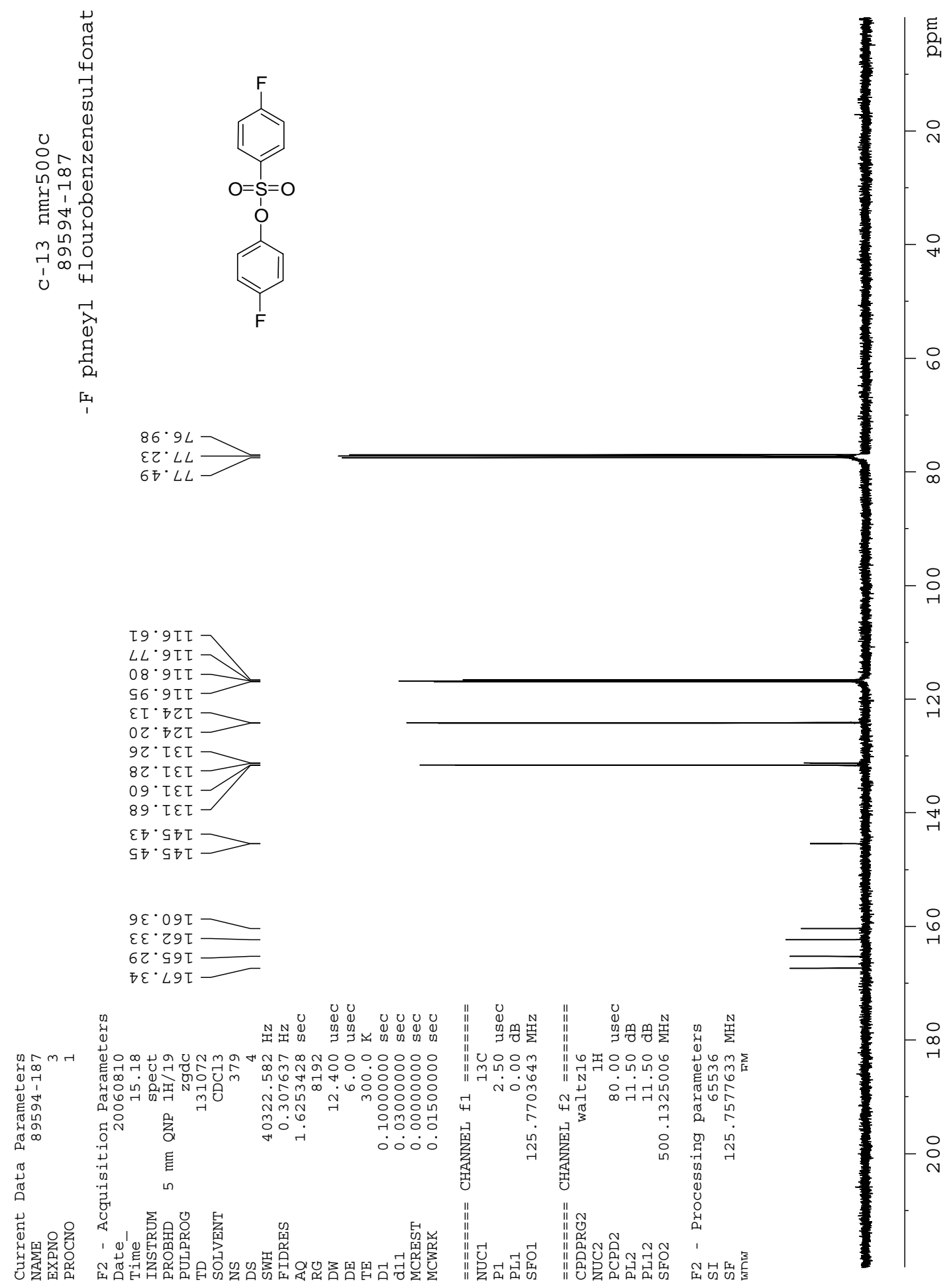

$9 \varepsilon \cdot 09 \tau \longrightarrow$

દย. 29 I

$6 \mathrm{r} \cdot 59 \mathrm{I}$

$\nabla \varepsilon \cdot \angle 9 I \longrightarrow$

$\stackrel{\infty}{\circ}$

$\stackrel{\circ}{\sim}$ 


\section{4-flurophenyl 4-fluorobenzenesulfonate (22)}
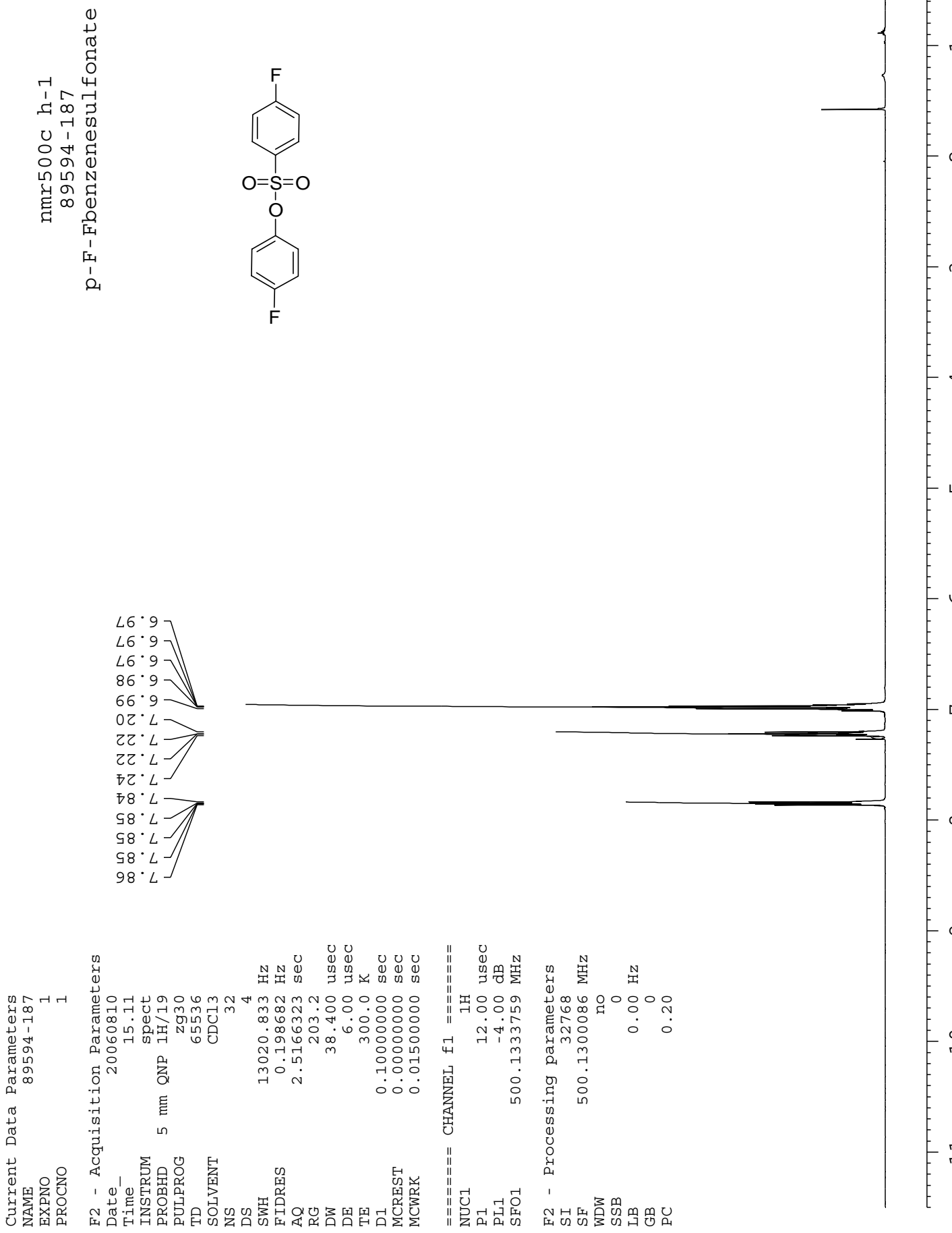

-6
-0
-
-7
-7 
pyridin-3-yl 4-fluorobenzenesulfonate (23)
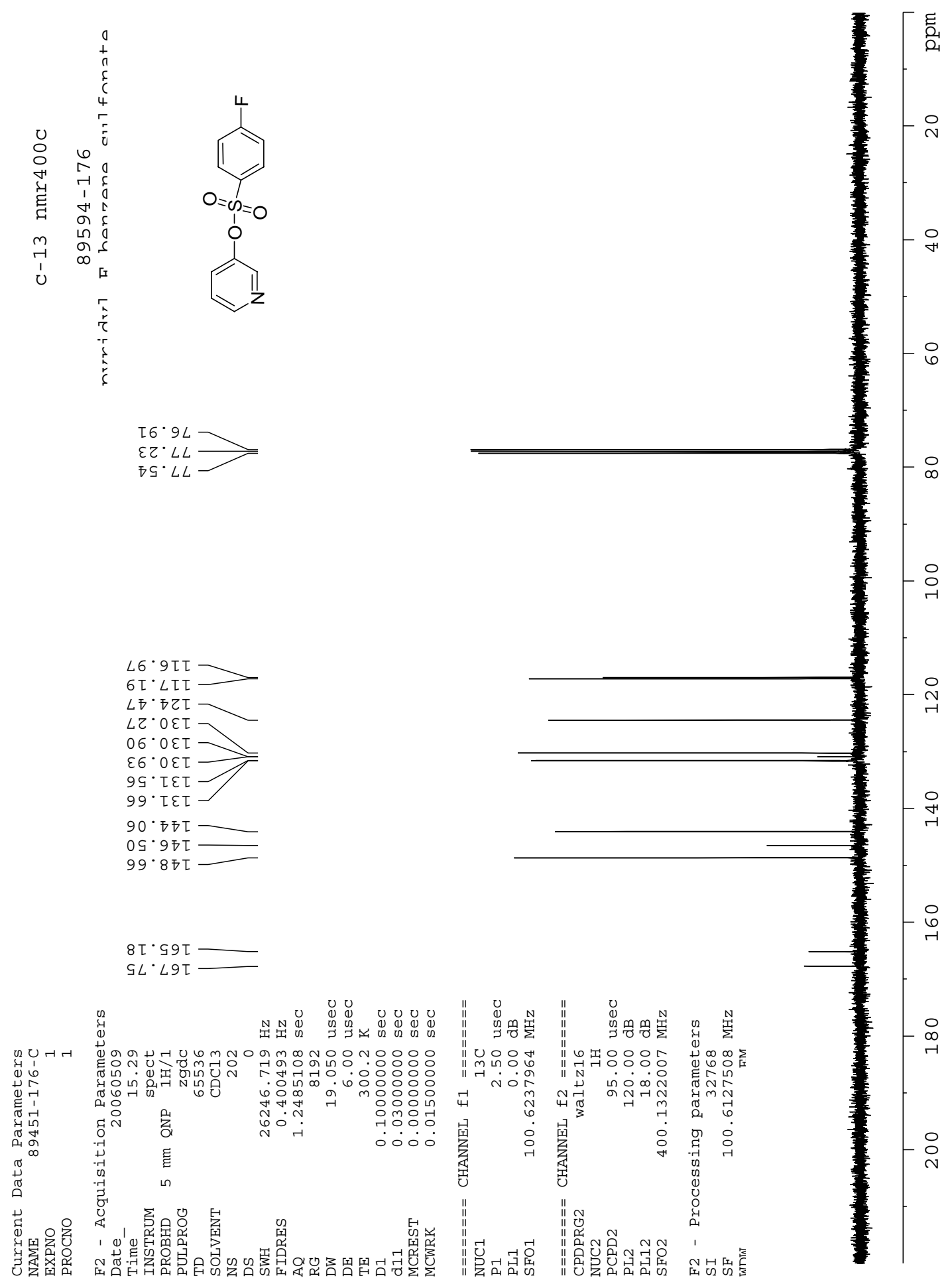
pyridin-3-yl 4-fluorobenzenesulfonate (23)

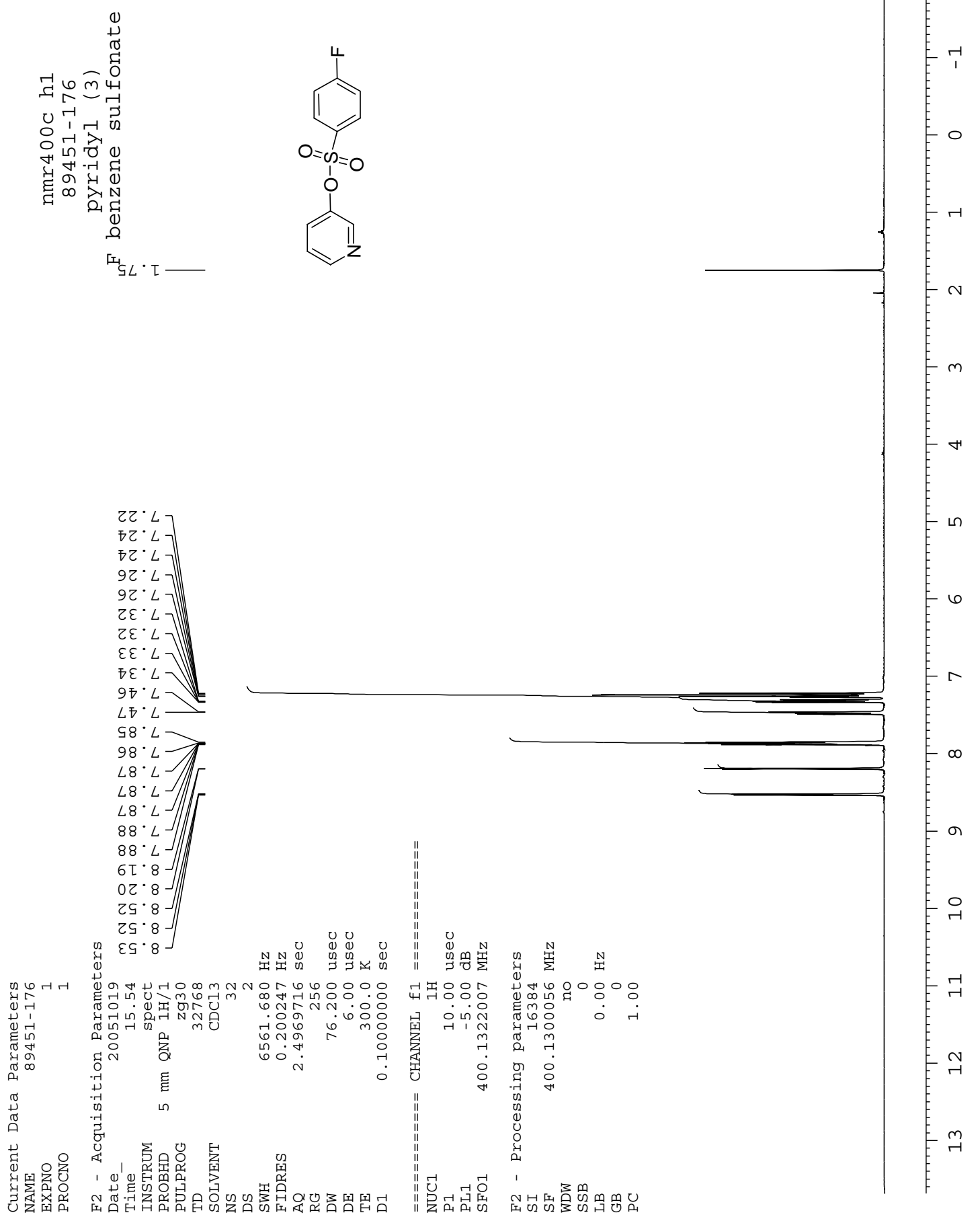




\section{4-methylphenyl 4-methylbenzenesulfonate (24)}
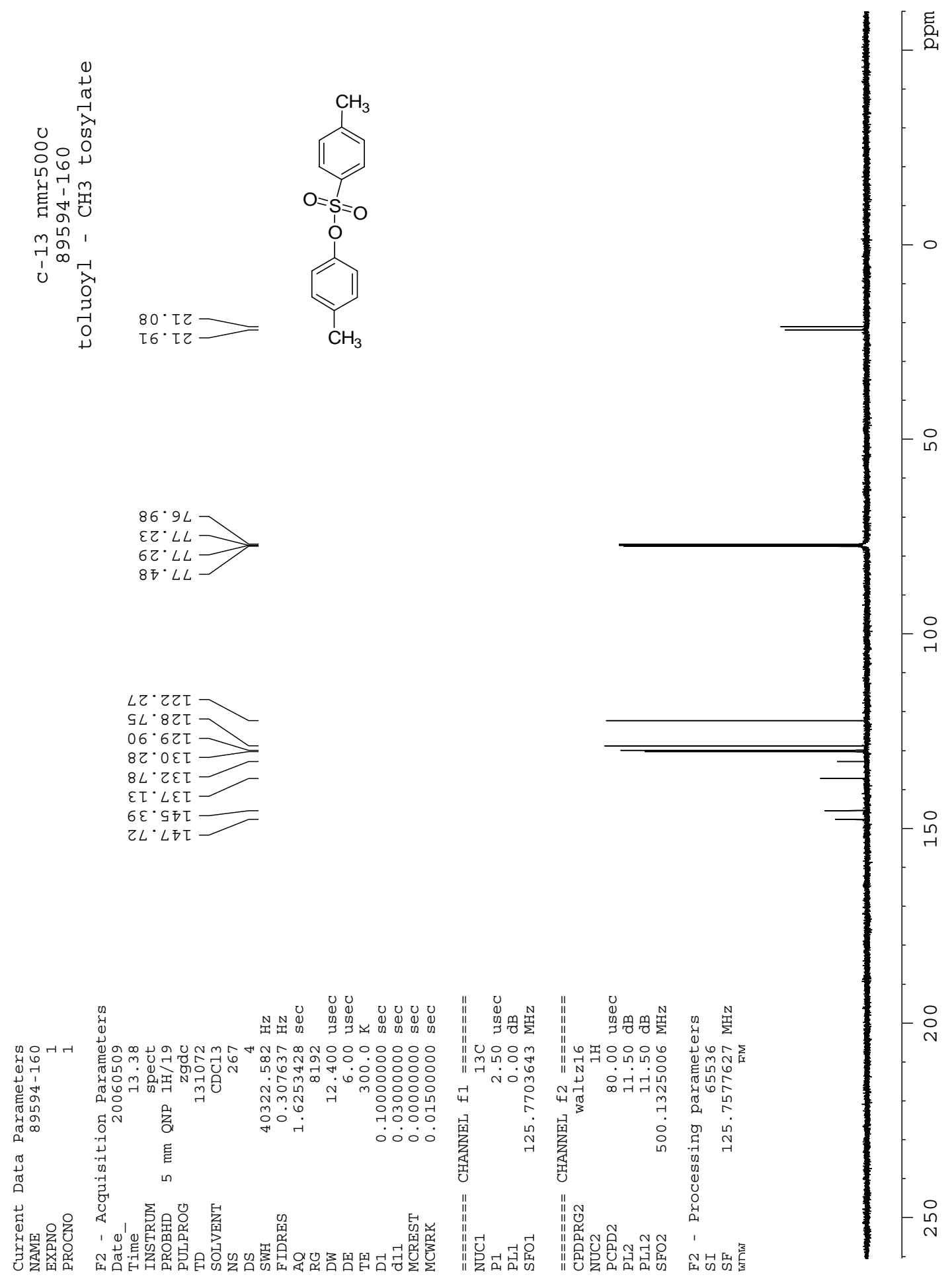


\section{4-methylphenyl 4-methylbenzenesulfonate (24)}

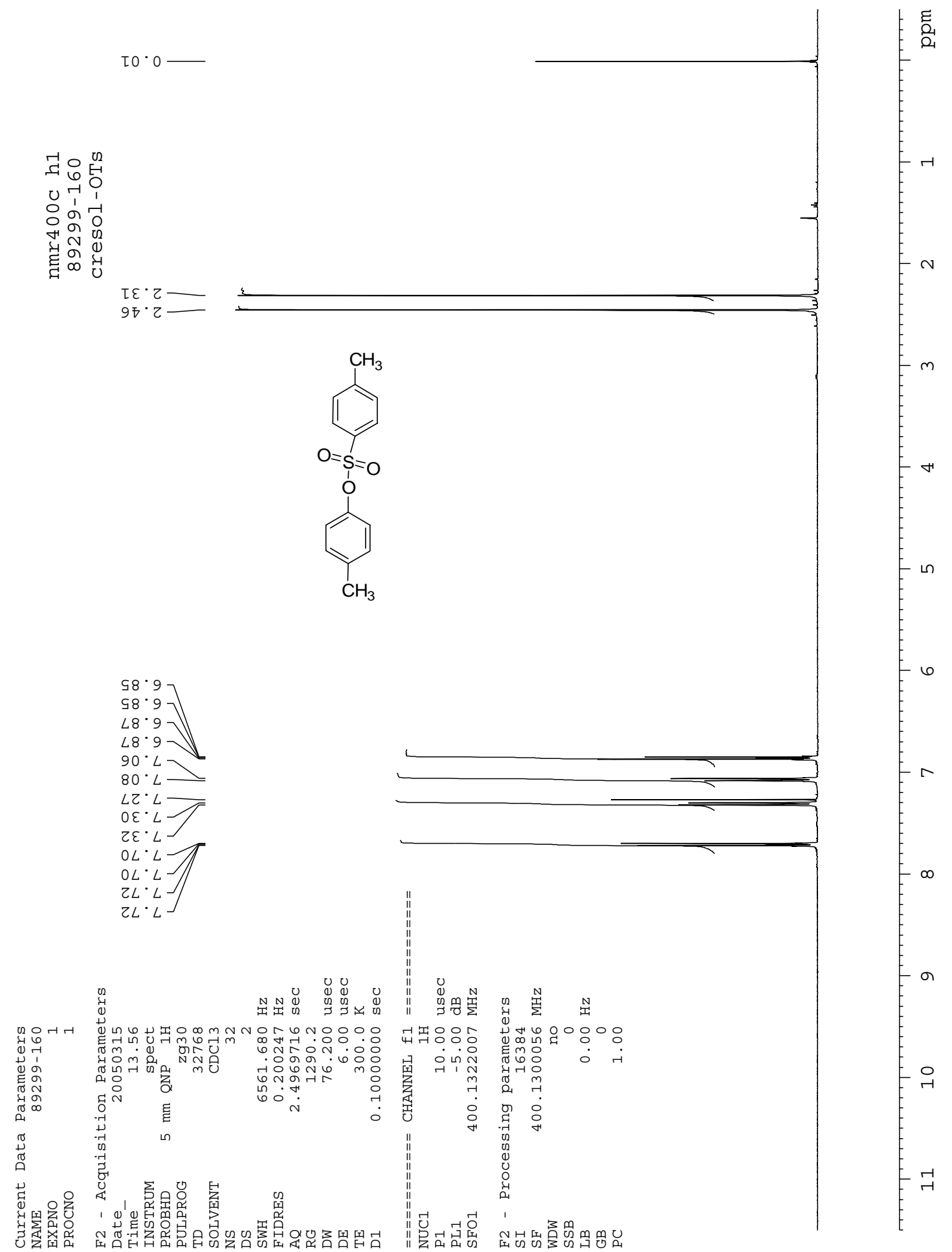

